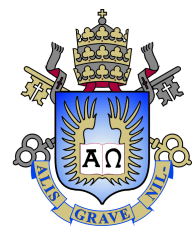

Marcelo Santos da Silva

\title{
Uma deformação de estrutura Poisson em variedade tórica e considerações cohomológicas
}

\section{Tese de Doutorado}

Tese apresentada como requisito parcial para obtenção do grau de Doutor pelo Programa de Pós-graduação em Matemáticada PUC-Rio.

Orientador: Prof. David Francisco Martínez Torres 

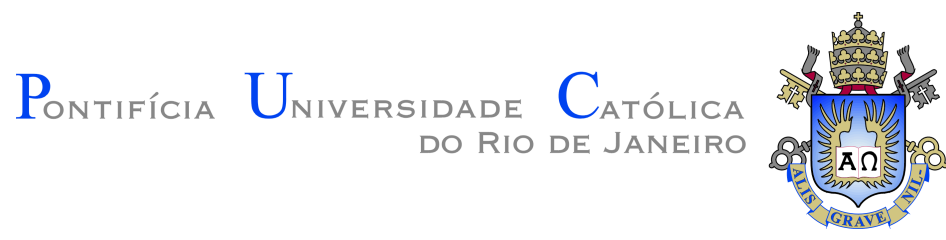

Marcelo Santos da Silva

\begin{abstract}
Uma deformação de estrutura Poisson em variedade tórica e considerações cohomológicas
\end{abstract}

Tese apresentada como requisito parcial para obtenção do grau de Doutor pelo Programa de Pós-graduação em Matemáticada PUC-Rio. Aprovada pela Comissão Examinadora abaixo assinada.

Prof. David Francisco Martínez Torres
Orientador
Departamento de Matemática - PUC-Rio

Prof. Alejandro Cabrera Instituto de Matemática - UFRJ

Profa. Alessia Mandini Instituto de Matemática e Estatística - UFF

Prof. Daniele Sepe Instituto de Matemática e Estatística - UFF

Prof. Ivan Struchiner Departamento de Matemática - USP

Prof. Marcos Craizer Departamento de Matemática - PUC-Rio

Prof. Pedro Walmsley Frejlich Departamento de Matematica Pura e Aplicada - UFRGS 
Todos os direitos reservados. É proibida a reprodução total ou parcial do trabalho sem autorização da universidade, do autor e do orientador.

\section{Marcelo Santos da Silva}

Licenciado em Matemática pela Universidade Federal de Pelotas (UFPel) em 2010. Bacharel em Matemática pela Universidade Federal do Rio Grande do Sul (UFRGS) em 2013. Mestre em Matemática pelo Instituto de Matemática Pura e Aplicada (IMPA) em 2015, com ênfase em Geometria.

Ficha Catalográfica

Silva, Marcelo Santos da

Uma deformação de estrutura Poisson em variedade tórica e considerações cohomológicas / Marcelo Santos da Silva; orientador: David Francisco Martínez Torres. - Rio de janeiro: PUC-Rio, Departamento de Matemática, 2021.

v., 63 f: il. color. ; $30 \mathrm{~cm}$

Tese (doutorado) - Pontifícia Universidade Católica do Rio de Janeiro, Departamento de Matemática.

Inclui bibliografia

1. Matemática - Teses. 2. Degeneração;. 3. Deformação;. 4. Cohomologia de Poisson;. 5. Variedade Tórica.. I. Torres, David Francisco Martínez. II. Pontifícia Universidade Católica do Rio de Janeiro. Departamento de Matemática. III. Título. 


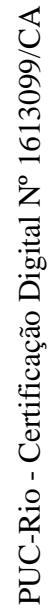

Para meus pais, Ione e Paulo 


\section{Agradecimentos}

Gostaria de agradecer literalmente a todos que de alguma forma contribuíram nesta jornada acadêmica. Por limitação de espaço fica aqui registrado os agradecimentos especiais.

Durante a licenciatura em matemática agradeço a Vinícius Beck a quem me influenciou ao caminho da luz, também conhecido como matemática pura.

No bacharelado agradeço a Matheus Bohrer, Eduardo Longa e Fábio Casula pelas ótimas tardes e muitas discussões divertidas de assuntos matemáticos e não-matemáticos.

No mestrado agradeço aos meus amigos Roberto Viveiros e Lucas Farias. Aqui um agradecimento ultra especial a minha amiga Letícia Alves a quem sempre me ajudou e fez toda a diferença na minha trajetória acadêmica. Aos três agradeço enormemente pelo acolhimento e pelas amizades.

Durante o doutorado agradeço a todos da sala 850 por tornar o dia a dia estudantil mais agradável. Em particular agradeço a Raimundo Leão, Fabíola Valéria e Aaron Aragon pelas discussões e ajudas matemáticas.

Agradeço ao meu orientador David Martínez Torres pela orientação, pelos incentivos, pela compreensão e instrução matemática, mas principalmente pela amizade.

Agradeço a PUC (instituição, professores, alunos e funcionários) pela bolsa de isenção, pela formação, pelo aprendizado e pelo acolhimento recebido.

Agradeço a CAPES pela bolsa de doutorado pela qual seria impossível executar tal feito. O presente trabalho foi realizado com apoio da Coordenação de Aperfeiçoamento de Pessoal de Nível Superior - Brasil (CAPES) - Código de Financiamento 001.

Agradeço aos meus irmãos Maicon e Márcio que como sempre são meus exemplos a seguir. Agradeço à minha cunhada Ana Paula pelo carinho e suporte como sempre me deu.

Por fim, agradeço às duas pessoas mais importantes que sempre me ajudaram e sempre me deram todo o amor e suporte que precisei, aos meus pais Ione e Paulo. 


\section{Resumo}

Silva, Marcelo Santos da; Torres, David Francisco Martínez. Uma deformação de estrutura Poisson em variedade tórica e considerações cohomológicas. Rio de Janeiro, 2021. 63p. Tese de Doutorado - Departamento de Matemática, Pontifícia Universidade Católica do Rio de Janeiro.

O estudo de deformações e degenerações de estruturas de Poisson ocupa posição especial dentro do marco clássico de análise de degenerações de estruturas geométricas. Nesta tese como resultado principal construímos uma deformação não trivial na qual a estrutura quadrática canônica do espaço projetivo complexo n-dimensional é limite contínuo de estruturas Kahlerianas. Além disso, como resultado segundário de estudos de deformações mostramos que uma estrutura Poisson invariante numa variedade tórica com número finito de folhas não pode ser exata na cohomologia Poisson. Nosso estudo também inclui considerações sobre cohomologia Poisson da estrutura quadrática canônica do espaço vetorial complexo n-dimensional.

\section{Palavras-chave}

Degeneração; Deformação; Cohomologia de Poisson; Variedade Tórica. 


\section{Abstract}

Silva, Marcelo Santos da; Torres, David Francisco Martínez (Advisor). A deformation of Poisson structure in toric variety and cohomological considerations. Rio de Janeiro, 2021. 63p. Tese de doutorado - Departamento de Matemática, Pontifícia Universidade Católica do Rio de Janeiro.

The study of deformations and degenerations of Poisson structures occupies a special position within the classical framework of analysis of degenerations of geometric structures. In this thesis as the main result we build a non-triavial deformation in which the canonical quadratic structure in $\mathbb{C P}^{n}$ is a continuous limit of Kahlerian structures. Furthermore, as a secondary result of deformation studies we have shown that an invariant Poisson structure in a toric variety with finite number of leaves cannot be exact in Poisson cohomology. Our study also includes considerations about Poisson cohomology of the canonical quadratic structure of $\mathbb{C}^{n}$.

\section{Keywords}

Degeneration; Deformation; Poisson Cohomology; Toric Manifold; 


\section{Sumário}

1 Introdução 10

2 Variedades Tóricas $\quad 15$

2.1 Introdução as Variedades Tóricas $\quad 15$

$\begin{array}{ll}2.2 \text { Variedades Tóricas Associadas a Semigrupos } & 16\end{array}$

$\begin{array}{lll}2.3 & \text { Fans } & 17\end{array}$

2.4 Variedades Tóricas Associadas a Fans 18

2.5 Fans Associados a Polítopos 20

2.6 Descrição Cartas-Tóricas (Poliedros) 22

3 Cohomologia de Poisson $\quad 28$

3.1 Definição da Cohomologia de Poisson 28

3.2 Interpretação da Cohomologia de Poisson 29

3.3 Cohomologia de Poisson vs Cohomologia de De Rham 30

3.4 Não-exatidão da estrutura Poisson-tórica 31

3.5 Cohomologia Poisson e Cohomologia Invariante em $\mathbb{C} \quad 33$

3.5.1 Da cohomologia para cohomologia invariante 33

3.5.2 O complexo invariante e funções no quadrante positivo 40

4 Deformação $\quad 46$

4.1 Problema Geral 46

4.2 Construção via Delzant 47

4.3 Construção via Arlo Caine 49

$\begin{array}{lll}4.4 & \text { Deformação } & 50\end{array}$

4.5 Função $\xi(t)$ e Interpretação Geométrica $\quad 56$

4.6 Poisson Comutativo 58 


\section{Lista de figuras}

$\begin{array}{lll}\text { Figura 2.1 } & \text { Fan } & 19\end{array}$

Figura 2.2 Polítopo e Fan 23

Figura 2.3 Polítopo de Delzant 25

Figura 4.1 Diagrama das ações 49

Figura 4.2 Diagrama comutativo $\quad 51$

Figura 4.3 Dilatação $\quad 52$

Figura 4.4 Diagrama comutativo $\quad 54$

Figura 4.5 Conjuntos de níveis 55 


\section{Introdução}

Mecânica é essencialmente a área da física que estuda o movimento. Chamamos de mecânica clássica ou mecânica newtoniana aos conceitos físicos empregados e aos métodos matemáticos para descrever o movimento dos corpos "macroscópicos" sob a influência de um sistema de forças, ou mais precisamente, que obedecem as leis de Newton para o movimento. Existem duas abordagens alternativas e equivalentes a mecânica clássica nas quais enfatizam a energia do sistema mais do que o sistema de forças, são elas a mecânica lagrangiana e mecânica hamiltoniana. Por vezes chamamos essas abordagens de mecânica analítica. Enquanto que mecânica lagrangiana é baseada em princípios variacionais e generaliza mais diretamente para o contexto relativístico geral a mecânica hamiltoniana baseia-se diretamente no conceito de energia e está mais intimamente ligado à mecânica quântica.

Sabemos que variedades simpléticas fornecem o cenário natural para a formulação hamiltoniana das equações diferenciais ordinárias da mecânica clássica, com o exemplo primário de variedade simplética sendo o fibrado cotangente $T^{*} \mathbb{R}^{n}$ de coordenadas globais $(q, p)$ equipado com a forma simplética canônica $d q \wedge d p$. Além disso, como uma generalização de estruturas simpléticas, as estruturas de Poisson aparecem naturalmente na descrição da dinâmica reduzida de sistemas mecânicos com simetrias. O estudo de tais estruturas, ou melhor, da geometria de Poisson é um assunto antigo que remonta à descoberta dos colchetes de Poisson. Teve um rápido desenvolvimento nas últimas décadas devido a fortes conexões com a teoria da quantização de deformação, teoria da singularidade, teoria de Lie, sistemas completamente integráveis e geometria complexa generalizada. São esses muitos links que tornam a geometria de Poisson hoje em dia um assunto central e o que explica porque os avanços na geometria de Poisson estão fadados a ter um impacto profundo em várias áreas diferentes.

Matematicamente, estruturas de poisson, ou ainda, variedades Poisson, possuem a propriedade de que em cada ponto são localmente isomorfas a uma soma direta de uma forma simplética (chamada de parte regular) com uma estrutura de Poisson "transversal" (chamada de parte singular) na qual se degenera no ponto em questão. Mais do que isso cada estrutura Poisson induz 
uma foliação singular cujas folhas são simpléticas. Weinstein em seu artigo [1] explicita que esta propriedade de ser suavemente decomposta em variedades simpléticas de diferentes dimensões parece tornar as variedades de Poisson uma estrutura apropriada para estudar um fenômeno que é bastante comum em mecânica: se um sistema mecânico é modelado por uma variedade simplética, então quando um parâmetro no sistema atinge um valor limite (geralmente 0 ou $\infty)$, o sistema limite também tem uma formulação simplética, mas com menos graus de liberdade. Exemplos de tais situações são:

1. o problema restrito de 3 corpos na mecânica celeste ;

$$
(\text { massa } \rightarrow 0)
$$

2. o limite do centro de orientação para uma partícula em um campo eletromagnético;

$($ carga/ massa $\rightarrow \infty)$

3. o limite de vórtices discretos no movimento de fluidos incompressíveis;

(concentração de vorticidade $\rightarrow \infty$ )

4. o limite clássico da mecânica quântica;

$(\hbar \rightarrow 0)$

Também há exemplos em que o número de graus de liberdade permanece o mesmo, mas a estrutura global de uma variedade simplética de grupos muda, como no limite newtoniano da relatividade especial $(c \rightarrow \infty)$; estes também devem ser acessíveis para estudar em termos de estruturas de Poisson.

Como vemos é rotineiro situações físicas onde a estrutura, no caso Poisson ou simplética, depende de um ou mais parâmetros, da mesma forma situações onde a "configuração limite" surge de forma natural e precisa ser estudada física e matematicamente. Mais precisamente, esses dois cenários tratam-se de deformações e degenerações de estruturas, nos exemplos citados anteriormente de deformações/degenerações de formas simpléticas. Do ponto de vista da geometria se considerarmos uma variedade arbitrária $M$ e formos estudar os espaços de estruturas Simplética/Poisson, $\operatorname{Sympl}(M) / \operatorname{Poisson}(M)$, bem como todas "configurações limites" parece um tanto audacioso demais, tamanha generalidade do problema. Para ilustrar essas díficuldades se restringirmos, por exemplo, as deformações ao contexto infinitesimal este problema passa a ser o estudo dos grupos de cohomologia de Poisson de $M$, para uma determinada estrutura $\Pi \in \operatorname{Poisson}(M)$. Ainda assim o problema continua geral e complexo demais, pois são poucas variedades de Poisson (não simpléticas) para as quais 
a cohomologia de Poisson foi computado e mesmo quando $M$ é compacta determinar se tais grupos têm dimensão finita ou não já é um problema difícil e em aberto para a maioria das estruturas de Poisson que conhecemos.

Mais geralmente, o estudo de deformações/degenarações são um marco clássico dentro da análise do espaço de estruturas geométricas. Em outras palavras, saber o que está no limite de tais espaços de estruturas podem trazer entendimento mais profundo tanto sobre a variedade em questão quanto sobre as próprias estruturas. Uma situação deste tipo é quando a estrutura na fronteira, ou configuração limite como chamamos anteriormente, pode codificar informações suficientes para reconstruir a estrutura original ou, pelo menos, definir invariantes. Um exemplo paradigmático é o caso das estruturas de contato em variedades compactas: elas degeneram em decomposições de livro aberto com folha Weinstein e monodromia simplética. Inversamente, uma tal decomposição de livro aberto é suficiente para reconstruir um segmento/raio de estruturas de contato que degenera nessa decomposição. Além disso, em dimensão três, pode-se extrair um invariante de contato através de uma certa construção em teoria de calibre.

Um outro ponto a ser destacado é que o estudo das estruturas degeneradas podem ser importantes por si mesmas, ou melhor, ter uma família de estruturas geométricas pode ser útil no estudo da estrutura degenerada. Um exemplo deste princípio, já na temática dessa tese, é o de uma estrutura simplética que degenera em uma estrutura Poisson, em que se criam novas folhas simpléticas. Uma vantagem aqui da perspectiva Poisson é que podemos ver esse fenômeno como uma família de estruturas Poisson a um parâmetro, evitando assim questões de convergência para a estrutura degenerada. Nesse sentido, pode-se também mudar de perspectiva, e pensar em uma deformação da estrutura Poisson na direção de uma estrutura.

Vamos a dois exemplos onde a extração uma deformação simplética trouxe resultados interessantes. Recentemente mostrou-se que as estruturas $b^{2 m}$-Poisson em variedades compactas são limites de estruturas simpléticas. Mais precisamente, um bivetor Poisson $\Pi$ sobre uma variedade $M^{2 n}$ é dita $b^{2 m}$-simplética quando for simplética sobre o complemento de uma hipersuperfície $Z$ e tiver forma canônica de Darboux "simples" nos pontos de $Z$. Em [5] foi mostrado que neste caso quando a variedade é compacta existe família de formas simpléticas $\omega_{\epsilon}$ na qual coincide com a forma $b^{2 m}$-simplética $\Pi$ fora de uma $\epsilon$-vizinhança de $Z$ e que essa família de bivetores converge na topologia $C^{2 k}$ para a estrutura Poisson em questão.

Uma das questões mais fundamentais em geometria simplética é determinar as restrições topológicas para uma variedade admitir uma estrutura 
simplética. Nesse espírito, o teorema mencionado, ou ainda a extração de uma deformação simplética, implica que qualquer variedade compacta admitindo uma estrutura $b^{2 m}$-simplética também admite uma estrutura simplética e, portanto, todas as restrições topológicas para uma variedade ser simplética também se aplicam a uma variedade admitindo um estrutura $b^{2 m}$-simplética. Mais do que isso, neste contexto em particular o objetivo ao introduzir essas famílias é que, ao tentar definir os análogos " $b^{m "}$ de uma série de invariantes básicos de variedades simpléticas e simpléticas dobradas (folded symplectic), como volume simplético, variedades $G$ hamiltonianas, politopos e medidas de Duistermaat-Heckman, encontramos uma série de "infinitos" frustrantes que são difíceis de interpretar ou eliminar, como é o caso/exemplo em [3]. Ferramentas como dessingularização são eficazes para contornar esse problema dos "infinitos".

Outro exemplo a ser mencionado é a estrutura de Bruhat-Poisson da órbita coadjunta regular/variedade bandeira completa $K / T$, essa por sua vez é mostrada ser uma degeneração de estruturas simpléticas [6]. Com essa degeneração em estruturas simpléticas foi possível calcular parte da homologia Poisson da estrutura Bruhat-Poisson.

Apresentada motivações, problemas e implicações de natureza física e geométrica sobre a temática deformações e degenerações, fica estabelecida a necessidade de tais estudos. Nessa tese como resultado principal construímos uma deformação na qual a estrutura quadrática canônica do espaço projetivo complexo $\mathbb{C P}^{n}$ é limite contínuo de estruturas Kahlerianas. Mais do que isso, provamos:

Teorema 1.1 Existe uma deformação não-trivial da estrutura quadrática canônica de $\mathbb{C P}^{n}$ na qual é limite contínuo de estruturas kahlerianas. Além disso, mostra-se que a ação do toro $\mathbb{T}^{n}$ em $\mathbb{C P}^{n}$ é hamiltoniana para cada uma dessas estruturas kahlerianas.

Como peça chave para a demonstração desse resultado nos utilizamos do fato que o espaço projetivo pode ser obtido via duas construções já sistematizadas, uma via GIT baseada na construção de Arlo Caine em [7] e a outra via teorema de Delzant e redução simplética. A estratégia baseia-se essencialmente em trabalhar com levantamentos a $\mathbb{C}^{n+1}$. Construímos uma combinação convexa $\Pi_{t}$ entre os bivetores quadrático canônico e o bivetores constante canônico, indexado por um $t \in[0,1]$ (com essa orientação). Nesse ponto verifica-se que para cada $t>0$ a estrutura em questão é simplética, além disso, a ação do toro $\mathbb{S}^{1}$ em $\left(\mathbb{C}^{n+1} \backslash\{0\}, \omega_{t}=\left(\Pi_{t}\right)^{-1}\right)$ é hamiltoniana. Utilizando-se da redução simplética e suas propriedades construímos família 
de bivetores a dois parâmetros em $\mathbb{C}^{n+1}$ nas quais descem a estruturas kahlerianas em $\mathbb{C P}^{n}$. Através de argumentos geométricos extraímos subfamília $\hat{\pi}_{t}$ a 1-parâmetro na qual converge a estrutura quadrática.

Como resultado secundário e independente de estudos de deformações mostramos que uma estrutura Poisson invariante numa variedade tórica com número finito de folhas não pode ser exata na cohomologia Poisson. Mais precisamente:

Teorema 1.2 Sejam $\Delta \subset \mathbb{R}^{n}$ polítopo de Delzant, $\mathcal{F}$ fan dual a $\Delta$ e $X=X_{\Delta}$ variedade tórica diferenciável associada. Se $\Pi \in \mathcal{X}^{2}(X)^{T_{\mathbb{C}}}$ estrutura Poisson $T$-invariante cujas folhas são exatamente as T-órbitas. Então $[\Pi] \neq 0$ em $H^{2}(X, \Pi)$, ou seja, П não é exata na cohomologia de Poisson por ela induzida.

A prova exige construções do capítulo II juntamente com resultados de Nakanishi [8]. Além disso, ainda considerando a estrutura quadrática canônica de $\mathbb{C}^{n}$ :

$$
\Pi_{s t d}=\sum_{j=1}^{n} \frac{\partial}{\partial z_{j}} \wedge \frac{\partial}{\partial \bar{z}_{j}}
$$

calculamos parte da cohomologia de Poisson de $\mathbb{C}^{2}$. Mostramos que:

Teorema 1.3 A cohomologia top $H_{P i_{s t d}}^{4}\left(\mathbb{C}^{2}\right)$ é isomorfa a $\mathbb{R}^{4}$.

onde a estratégia fundamental foi reduzir o cálculo da cohomologia para cohomologia invariante. Posteriormente mostrar que tais complexos Poisson invariante formam um módulo livre sobre as funções invariantes. 


\section{2}

\section{Variedades Tóricas}

Neste capítulo vamos relembrar algumas definições e construções básicas sobre variedades tóricas. O objetivo aqui será apresentar os diferentes objetos que descrevem uma variedade tórica e as relações entre essas descrições. No final será contruido um atlas holomorfo associado a cada politopo de Delzant, construção essa necessária ao próximo capítulo.

\section{1}

\section{Introdução as Variedades Tóricas}

Para estabelecer notações e nomenclaturas:

\section{Definição 2.1}

- Chamamos de toro algébrico (complexo) n-dimensional $\mathbb{T}_{\mathbb{C}}$ ao conjunto $\left(\mathbb{C}^{*}\right)^{n}$ munido com a multiplicação usual dos números complexos;

- Uma ação (tórica) de um toro algébrico $\left(\mathbb{C}^{*}\right)^{n}$ sobre uma variedade algébrica $X$ é uma ação induzida por um homomorfismo de grupos $\psi:\left(\mathbb{C}^{*}\right)^{n} \longrightarrow \operatorname{ISO}(X) ;$

- Uma variedade tórica é uma variedade algébrica irredutivel X equipada com uma ação de um toro algébrico possuindo uma órbita aberta e densa em $X$.

Geralmente inclui-se na definição de variedade tórica a propriedade de normalidade, ou seja, que o anel de coordenadas $\mathbb{C}[X]$ seja integralmente fechado a seu corpo de funções regulares $\mathbb{C}(X)$, i.e., se $P \in \mathbb{C}(X)$ é raiz de algum polinômio mônico com coefícientes em $\mathbb{C}[X]$, então $P \in \mathbb{C}[X]$.

\section{Exemplo 2.2}

1. $\left(\mathbb{C}^{*}\right)^{n}$ e $\mathbb{C}^{n}$ são evidentemente variedades tóricas;

2. Seja $C$ a curva plana $\mathbf{V}\left(X^{3}-Y^{2}\right) \subseteq \mathbb{C}^{2}$. $C$ é irredutível uma vez que $X^{3}-Y^{2} \in \mathbb{C}[X, Y]$ é irredutível. $A$ ação $\mathbb{C}^{*} \times C \longrightarrow C$ dada por

$$
t \cdot(x, y)=\left(t^{2} x, t^{3} y\right)
$$


é regular e possui órbita $\mathbb{C}^{*} \cdot(1,1)=C \backslash\{0\}$ aberta e densa em $C$. Neste caso $C$ é variedade tórica, porém não é normal.

3. Seja $A=\left\{\lambda^{(1)}, \ldots, \lambda^{(k)}\right\} \subset \mathbb{Z}^{n}$ conjunto não-vazio. Induzimos uma ação de $\left(\mathbb{C}^{*}\right)^{n}$ em $\mathbb{C}^{k}$ relativo a $A$ definida por:

$$
w \cdot\left(z_{1}, \ldots, z_{k}\right)=\left(w^{\lambda^{(1)}} z_{1}, \ldots, w^{\lambda^{(k)}} z_{k}\right), \text { para todo } w \in\left(\mathbb{C}^{*}\right)^{n}
$$

Seja $Y_{A}$ o fecho de $\left(\mathbb{C}^{*}\right)^{n} \cdot(1, \ldots, 1)$. Então $Y_{A}$ é variedade tórica;

4. Analogamente seja $A=\left\{\lambda^{(1)}, \ldots, \lambda^{(k)}\right\} \subset \mathbb{Z}^{n}$ conjunto não-vazio. Induzimos uma ação de $\left(\mathbb{C}^{*}\right)^{n}$ em $\mathbb{C P}^{k-1}$ relativo a $A$ definida por:

$$
w \cdot\left[z_{1}: \ldots: z_{k}\right]=\left[w^{\lambda^{(1)}} z_{1}: \ldots: w^{\lambda^{(k)}} z_{k}\right], \text { para todo } w \in\left(\mathbb{C}^{*}\right)^{n}
$$

Seja $X_{A}$ o fecho de $\left(\mathbb{C}^{*}\right)^{n} \cdot[1: \cdots: 1]$. Então $X_{A}$ é variedade tórica;

Um aspecto bastante importante de variedades tóricas que as fazem ser um tópico especial em geometria algébrica se deve ao fato de possuirem fortes conexões com outros objetos e até mesmo com outras áreas da matemática, como por exemplo cones, poliedros, fans, etc, ou ainda, combinatória, álgebra comutativa, geometria simplética e topologia. Vamos apresentar algumas dessas conexoes de maneira breve, suficientes para as próximas seções.

\section{2}

\section{Variedades Tóricas Associadas a Semigrupos}

Seja $S$ um semigrupo abeliano. Sua álgebra de semigrupo sobre $\mathbb{C}$ é definida por

$$
\mathbb{C}[S]:=\left\{\sum_{i=1}^{k} a_{i} Z^{\sigma_{i}} \mid k \in \mathbb{N}, a_{i} \in \mathbb{C}, \sigma_{i} \in S\right\}
$$

onde a multiplicação é definida por $Z^{\sigma} Z^{\sigma^{\prime}}=Z^{\sigma+\sigma^{\prime}}$, com $\sigma, \sigma^{\prime} \in S$, e estendida distributivamente. Em particular se $G \subset S$ é um conjunto de geradores como semigrupo, então $\left\{Z^{\sigma} \mid \sigma \in G\right\}$ forma um conjunto de geradores para $\mathbb{C}[S]$ como $\mathbb{C}$-álgebra.

\section{Exemplo 2.3}

1. Se $S=\left(\mathbb{Z}_{\geqq 0}\right)^{n}$, então $\mathbb{C}[S]=\mathbb{C}\left[z_{1}, \ldots, z_{n}\right]$ é a $\mathbb{C}$-álgebra de polinômnios em $n$ variáveis.

2. Se $S=\mathbb{Z}^{n}$, então $\mathbb{C}[S]=\mathbb{C}\left[z_{1}^{ \pm}, \ldots, z_{n}^{ \pm}\right]=\mathbb{C}\left[z_{1}, \ldots, z_{n}\right]_{z_{1} \cdots z_{n}}$ a $\mathbb{C}$-álgebra dos polinômios de Laurents em n varieáveis. 
Proposição 2.4 Seja $S \subset \mathbb{Z}^{n}$ um semigrupo finitamente gerado. Então $\operatorname{Spec}_{m}(\mathbb{C}[S])$, munida da topologia de Zariski, é uma variedade tórica afim.

Proof. [12]

Proposição 2.5 Qualquer variedade tórica afim é equivalente a uma variedade tórica afim da forma $\operatorname{Spec}_{m}(\mathbb{C}[S])$ para algum semigrupo $S \subset \mathbb{Z}$ finitamente gerado.

Proof. [12]

\section{3}

Fans

\section{Definição 2.6}

- Um cone (poliedral convexo) em $\mathbb{R}^{n}$ é um conjunto da forma:

$$
C=\left\{a_{1} v_{1}+\cdots+a_{r} v_{r} \in \mathbb{R}^{n} \mid a_{1}, \ldots, a_{r} \geqslant 0\right\}
$$

onde o conjunto finito $\left\{v_{1}, \ldots, v_{r}\right\}$ é chamado de geradores do cone $C$;

- Um cone $C \subset \mathbb{R}^{n}$ é dito racional se possui um conjunto $\left\{v_{1}, \ldots, v_{k}\right\} \subset$ $\mathbb{Z}^{n}$ de geradores. Se, além disso, tal conjunto $G$ for parte de uma $\mathbb{Z}$-base de $\mathbb{Z}^{n}$ dizemos que $C$ é suave;

- O dual do cone $C \subset \mathbb{R}^{n}$ é definido por:

$$
C^{*}=\left\{f \in\left(\mathbb{R}^{n}\right)^{*} \mid f(x) \geqslant 0, \forall x \in C\right\}
$$

- Uma face do cone $C \subset \mathbb{R}^{n}$ é: ou o próprio $C$ ou um conjunto $C \cap H_{f}$, onde $f \in C^{*} \backslash\{0\}$ e $H_{f}:=\left\{x \in \mathbb{R}^{n} \mid f(x)=0\right\} ;$

- Um cone $C \subset \mathbb{R}^{n}$ é chamado de fortemente convexo se $\{0\}$ for uma face de $C$, ou equivalentemente, se $C \cap(-C)=\{0\}$;

Notemos que se $C \subset \mathbb{R}^{n}$ for cone, então sob as operações de adição e multiplicação por escalar usuais em $\mathbb{R}^{n}$, temos que $C$ é semigrupo. Para evidenciar esse fato denotaremos $C$ por $S_{C}$.

Proposição 2.7 Sejam $C$ e $C^{\prime}$ cones racionais. Se $C$ for uma face de $C^{\prime}$, então o mapa induzido $\operatorname{Spec}_{m}\left(\mathbb{C}\left[S_{c}\right]\right) \longrightarrow \operatorname{Spec}_{m}\left(\mathbb{C}\left[S_{C^{\prime}}\right]\right)$ é injeção aberta para a topologia de Zariski.

Proof. [12] 
Definição 2.8 Um fan em $\mathbb{R}^{n}$ é uma coleção finita $\mathcal{F}=\left\{C_{\lambda}\right\}_{\lambda \in \Gamma}$ de cones racionais fortemente convexos satisfazendo:

- Toda face de todo cone $C \in \mathcal{F}$ pertente a $\mathcal{F}$;

- A interseção de quaisquer 2 cones de $\mathcal{F}$ é uma face comum de ambos cones;

Um fan $\mathcal{F}$ é dito suave se todos seus cones são suaves. O suporte de $\mathcal{F}$ é definido como a união $|\mathcal{F}|$ de seus cones. Um fan $\mathcal{F}$ em $\mathbb{R}^{n}$ é dito completo quando $|\mathcal{F}|=\mathbb{R}^{n}$.

\section{4}

\section{Variedades Tóricas Associadas a Fans}

A partir da última proposição e dos resultados precedentes, fica bem visto que dado um fan $\mathcal{F}$ em $\mathbb{R}^{n}$ temos associados a cada um de seus cone $C \in \mathcal{F}$ uma variedade tórica afim $X_{C}:=\operatorname{Spec}_{m}\left(\mathbb{C}\left[S_{C}\right]\right)$, na qual duas dessas variedades afins $X_{C}$ e $X_{C^{\prime}}$ são identificadas vias injeções abertas $X_{C \cap C^{\prime}} \hookrightarrow X_{C}$ e $X_{C \cap C^{\prime}} \hookrightarrow X_{C^{\prime}}$. Definimos assim a variedade algébrica:

$$
X_{\mathcal{F}}:=\left(\bigcup_{C \in \mathcal{F}} X_{C}\right) / \sim
$$

A ação tórica de cada $X_{C}$ é compatível com a identificação induzida nas faces. Desta forma há uma ação tórica bem definida sobre $X_{\mathcal{F}}$ na qual $X_{\{0\}}=\operatorname{Spec}_{m}\left(\mathbb{C}\left[Z_{1}^{ \pm 1}, \ldots, Z_{n}^{ \pm 1}\right]\right)=\left(\mathbb{C}^{*}\right)^{n} \hookrightarrow X_{\mathcal{F}}$ possui imagem aberta e densa. Desta forma, a cada fan $\mathcal{F}$ de $\mathbb{R}^{n}$ induz uma $\left(\mathbb{C}^{*}\right)^{n}$-variedade tórica.

$$
\begin{aligned}
\text { ffans } \left.\mathcal{F} \text { em } \mathbb{R}^{n}\right\} \longrightarrow & \left\{\left(\mathbb{C}^{*}\right)^{n} \text {-Variedades Tóricas }\right\} \\
\mathcal{F} \mapsto X_{\mathcal{F}} &
\end{aligned}
$$

\section{Exemplo 2.9}

1. Sejam $\left\{e_{1}, \ldots, e_{n}\right\}$ a base canônica de $\mathbb{R}^{n}$ e $e_{0}:=-e_{1}-\cdots-e_{n}$. Para cada coleção $I=\left\{e_{i_{1}}, \ldots, e_{i_{k}}\right\} \subset\left\{e_{0}, e_{1}, \ldots, e_{n}\right\}$ definimos $C_{I}=C_{i_{1} \ldots i_{k}}$ como o cone fortemente convexo em $\mathbb{R}^{n}$ gerado pelo conjunto $\left\{e_{i_{1}}, \ldots, e_{i_{k}}\right\}$. É fácil ver que a seguinte coleção é um fan:

$$
\mathcal{F}:=\left\{C_{I}\right\}_{I \subset\left\{e_{0}, \ldots, e_{n}\right\}} \cup\{C\}
$$

onde $C=\{0\}$. As construções nos levam a $X_{\mathcal{F}}=\mathbb{C P}^{n}$. 
2. Seja $\left\{e_{1}, \ldots, e_{n}\right\}$ a base canônica de $\mathbb{R}^{n}$. Para cada $I=\left\{e_{i_{1}}, \ldots, e_{i_{k}}\right\} \subset$ $\left\{ \pm e_{1}, \ldots, \pm e_{n}\right\}$ definimos $C_{I}=C_{i_{1} \ldots i_{k}}$ como o cone fortemente convexo em $\mathbb{R}^{n}$ gerado pelo conjunto $\left\{e_{i_{1}}, \ldots, e_{i_{k}}\right\}$. A coleção $\mathcal{F}:=$ $\left\{C_{I}\right\}_{I \subset\left\{e_{0}, \ldots, e_{n}\right\}} \cup\{C\}$, onde $C=\{0\}$, forma um fan com $X_{\mathcal{F}}=\mathbb{C P}^{1} \times$ $\cdots \times \mathbb{C P}^{1}=\left(\mathbb{C P}^{1}\right)^{n}$

Uma vez que cada variedade tórica $X_{\mathcal{F}}$ é a colagem de variedades tóricas afins normais, resuta-se que $X_{\mathcal{F}}$ é normal tendo em vista que normalidade é uma propriedade local. A próxima proposição justica o porquê geralmente assumisse normalidade na definição de variedade tórica.

\section{Proposição 2.10 (Classificação das Variedades Tóricas Normais)} Qualquer variedade torica normal $X$ é equivalente a uma variedade tórica da forma $X_{\mathcal{F}}$ para algum fan em $\mathbb{R}^{n}$, onde $n$ é a dimensão do toro agindo em $X$.

Como uma consequencia imediata temos a equivalência:

$$
\left\{\text { fans } \mathcal{F} \text { em } \mathbb{R}^{n}\right\} \cong\left\{\left(\mathbb{C}^{*}\right)^{n} \text {-Variedades Tóricas Normais }\right\}
$$

Novamente, é por causa da natureza dessas classificações que usualmente adiciona-se normalidade ao conceito de variedádes tóricas.

Proposição 2.11 Seja $P$ a envoltória convexa em $\mathbb{R}^{n}$ do conjunto $A=$ $\left\{\lambda^{(1)}, \ldots, \lambda^{(k)}\right\} \subset \mathbb{Z}^{n}$ e seja $X_{A}$ a variedade tórica associada a A. Então existe uma bijeção entre as faces do politopo $P$ e as $\left(\mathbb{C}^{*}\right)^{n}$-órbitas em $X_{A}$ dadas por:

$$
\begin{aligned}
\left\{\begin{array}{c}
\text { faces do } \\
\text { politopo } P
\end{array}\right\} & \longrightarrow\left\{\begin{array}{c}
\left(\mathbb{C}^{*}\right)^{n} \text {-órbitas } \\
\mathrm{em} X_{A}
\end{array}\right\} \\
F & \longmapsto X^{0}(F)
\end{aligned}
$$

onde $X^{0}(F)=\left\{[u] \in X_{A} \mid \forall \lambda: u_{\lambda}=0 \Leftrightarrow \lambda \notin F\right\}$.

Proof. [12]

\section{Exemplo 2.12}

Sejam $A=\{(0,0),(1,0),(0,1)\} \subset \mathbb{Z}^{2}$ e $\Delta$ o politopo proveniente da envoltória convexa de $A$. Sabemos que associado a $A$ temos a variedade tórica $X_{A}=$ fecho de $\left\{\left[1: w_{2}: w_{3}\right] \in \mathbb{C P}^{2}: w_{1}, w_{2} \in \mathbb{C}^{*}\right\}=\mathbb{C P}^{2}$.
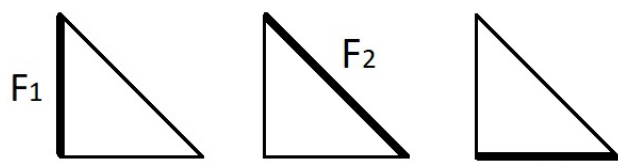

$\mathrm{F}_{3}$

Figura 2.1: Fan 
Vamos verificar a bijeção entre faces de $P$ e $\left(\mathbb{C}^{*}\right)^{2}$-órbitas de $X_{A}=\mathbb{C P}^{2}$. $O$ conjunto $A \subset \mathbb{Z}^{2}$ dá origem a uma ação tórica $\phi:\left(\mathbb{C}^{*}\right)^{2} \times \mathbb{C}^{3} \longrightarrow \mathbb{C}^{3}$ dada por $\left(w_{1}, w_{2}\right) \cdot\left(z_{1}, z_{2}, z_{3}\right)=\left(z_{1}, w_{1} z_{2}, w_{2} z_{3}\right)$ na qual age linearmente e induz a seguinte decomposição:

$$
\mathbb{C}^{3} \simeq \bigoplus_{\lambda \in Z^{n}} \mathbb{C}_{\lambda}^{3}
$$

$\lambda \in \mathbb{Z}^{2}$ temos $\mathbb{C}_{\lambda}^{3}:=\left\{z \in \mathbb{C}^{3}: w \cdot z=w^{\lambda} z, \forall w \in\left(\mathbb{C}^{*}\right)^{2}\right\}$.

$$
\begin{aligned}
\mathbb{C}_{(0,0)}^{3} & =\left\{z \in \mathbb{C}^{3}:\left(z_{1}, w_{1} z_{2}, w_{2} z_{3}\right)=\left(z_{1}, z_{2}, z_{3}\right), \forall w \in\left(\mathbb{C}^{*}\right)^{2}\right\}= \\
& =\mathbb{C} \times\{0\} \times\{0\} \\
\mathbb{C}_{(1,0)}^{3} & =\left\{z \in \mathbb{C}^{3}:\left(z_{1}, w_{1} z_{2}, w_{2} z_{3}\right)=w_{1}\left(z_{1}, z_{2}, z_{3}\right), \forall w \in\left(\mathbb{C}^{*}\right)^{2}\right\}= \\
& =\{0\} \times \mathbb{C} \times\{0\} \\
\mathbb{C}_{(0,1)}^{3} & =\left\{z \in \mathbb{C}^{3}:\left(z_{1}, w_{1} z_{2}, w_{2} z_{3}\right)=w_{2}\left(z_{1}, z_{2}, z_{3}\right), \forall w \in\left(\mathbb{C}^{*}\right)^{2}\right\}= \\
& =\{0\} \times\{0\} \times \mathbb{C}
\end{aligned}
$$

Logo $\mathbb{C}^{3}=\mathbb{C}_{(0,0)}^{3} \oplus \mathbb{C}_{(1,0)}^{3} \oplus \mathbb{C}_{(0,1)}^{3}$. As correspondências são:

- $F_{0}:=P \rightsquigarrow X^{0}\left(F_{0}\right)=\left\{\left[z_{1}: z_{2}: z_{3}\right] \in \mathbb{C P}^{2}: z_{1}, z_{2}, z_{3} \in \mathbb{C}^{*}\right\}$

- $F_{1} \rightsquigarrow X^{0}\left(F_{1}\right)=\left\{\left[z_{1}: 0: z_{3}\right] \in \mathbb{C P}^{2}: z_{1}, z_{3} \in \mathbb{C}^{*}\right\}$

- $F_{2} \rightsquigarrow X^{0}\left(F_{2}\right)=\left\{\left[0: z_{2}: z_{3}\right] \in \mathbb{C P}^{2}: z_{2}, z_{3} \in \mathbb{C}^{*}\right\}$

- $F_{3} \rightsquigarrow X^{0}\left(F_{3}\right)=\left\{\left[z_{1}: z_{2}: 0\right] \in \mathbb{C P}^{2}: z_{1}, z_{2} \in \mathbb{C}^{*}\right\}$

- $F_{12}:=\{(0,1)\} \rightsquigarrow X^{0}\left(F_{12}\right)=\left\{\left[0: 0: z_{3}\right] \in \mathbb{C P}^{2}: z_{3} \in \mathbb{C}^{*}\right\}$

- $F_{13}:=\{(0,0)\} \rightsquigarrow X^{0}\left(F_{12}\right)=\left\{\left[z_{1}: 0: 0\right] \in \mathbb{C P}^{2}: z_{1} \in \mathbb{C}^{*}\right\}$

- $F_{23}:=\{(1,0)\} \rightsquigarrow X^{0}\left(F_{12}\right)=\left\{\left[0: z_{2}: 0\right] \in \mathbb{C P}^{2}: z_{2} \in \mathbb{C}^{*}\right\}$

\section{5}

\section{Fans Associados a Polítopos}

O termo polítopo é hoje em dia um termo amplo que cobre uma vasta classe de objetos e várias definições aparecem na literatura matemática. Muitas dessas definições não são equivalentes entre si, resultando em diferentes conjuntos de objetos sendo chamados pelo mesmo nome de polítopos. Desta forma, antes de começarmos essa seção vamos a um esclarecimento de nomenclatura que será útil tanto nessa como na próxima seção. 
Definição 2.13 Um conjunto $\Delta \subset \mathbb{R}^{n}$ será chamado de polítopo se for descrito como a envoltória convexa de um número finito de pontos de $\mathbb{R}^{n}$. Nesse caso, podemos caracterizar $\Delta$ como a interseção finita (e minimal) de semiespaços $\bigcap_{i-1}^{k} f_{i}\left(\left(-\infty, a_{i}\right)\right)$, onde $f_{i}$ é função linear. Chamamos os vértices de 0 -faces, as arrestas de 1-faces, e assim por diante. As $(n-1)$-faces serão chamadas de facetas.

Podemos de forma natural associar a cada polítopo $\Delta$ um fan. Para isso, sejam $\Delta \subset \mathbb{R}^{n}$ um politopo e $f: \mathbb{R}^{n} \longrightarrow \mathbb{R}$ linear. Chamamos de face suporte de $\mathrm{f}$ ao conjunto $\operatorname{supp}_{\Delta}(f):=\{x \in \Delta: f$ tem mínimo em $x\}$. Se $F$ for uma face de $\Delta$, então definimos o cone:

$$
C_{F, \Delta}:=\left\{f \in\left(\mathbb{R}^{n}\right)^{*}: \operatorname{supp}_{\Delta}(f)=F\right\}
$$

Sem grandes dificuldades pode-se verificar que $C_{F, \Delta}$ é um cone fortemente convexo, conforme definido nas seções precedentes. Mais do que isso, pode-se checar que $\mathcal{F}_{\Delta}:=\left\{C_{F, \Delta}\right\}_{F}$ face de $\Delta$ forma um fan completo. Por $\Delta \subset \mathbb{R}^{n}$ e $\mathcal{F}_{\Delta}$ ser fan em $\left(\mathbb{R}^{n}\right)^{*}$, chamamos $\mathcal{F}_{\Delta}$ de fan dual a $\Delta$. Podemos ainda considerar o caso em que $\Delta$ possui a origem como ponto interior, assim definimos o polítopo dual:

$$
\Delta^{*}:=\left\{f \in \mathbb{R}^{n}: f(v) \geq-1, \forall v \in \Delta\right\}
$$

Algumas implicações imediatas são:

- $\Delta^{*} \subset\left(\mathbb{R}^{n}\right)^{*}$ é polítopo e suas faces geram os cones de $\mathcal{F}_{\Delta}$;

- $\Delta$ é racional, então $\Delta^{*}$ e $\mathcal{F}_{\Delta}$ são racionais;

- $\Delta$ é suave, então $\Delta^{*}$ e $\mathcal{F}_{\Delta}$ são suaves;

Devemos ter em mente que nem todos os fans provém de polítopos. Politopos que admitem o mesmo fan dual podem ser geometricamente diferentes, por exemplo, faces correspondentes podem ter diferentes volumes relativos, mas seus dados combinatórios são os mesmos.

Exemplo 2.14 Seja $\Delta \subset \mathbb{R}^{n}$ o n-simplex dado pela emvoltória convexa da base canônica $\left\{f_{1}, \ldots, f_{n}\right\}$ juntamnete com a origem $f_{0}:=0$. Identificamos $\mathbb{R}^{n} \simeq\left(\mathbb{R}^{n}\right)^{*}$ canônicamente. Notemos que para cada $j$ em $\{0,1, \ldots, n\}$ a faceta $F_{j}^{(n-1)}=\operatorname{Env} \operatorname{Conv}\left\{f_{0}, \ldots, \widehat{f}_{j}, \ldots, f_{n}\right\}$, onde o acento circunflexo indica omissão, está associado ao cone fortemente convexo 1-dimensional

$$
C_{F_{j}, \Delta}=\left\{\begin{array}{l}
\mathbb{R}_{\geqslant 0} f_{j}, \text { se } j=1, \ldots, n \\
\mathbb{R}_{\geqslant 0} e_{0}, \text { se } j=0
\end{array}\right.
$$


onde $e_{0}:=-f_{1}-\cdots-f_{n}$. Analisando o restante das faces de $\Delta$ podemos perceber que o fan dual associado $\mathcal{F}_{\Delta}$ é exatamente o fan do exemplo 1 da seção 2.4, ou seja, o politopo $\Delta$ está associado ao fan completo $\mathcal{F}_{\Delta}$ que por sua vez está associado a variedade tórica $X_{\mathcal{F}_{\Delta}}=\mathbb{C P}^{n}$.

\section{6}

\section{Descrição Cartas-Tóricas (Poliedros)}

Definição 2.15 Um polítopo $\Delta \subset \mathbb{R}^{d}$ é chamado de Delzant se satisfaz:

- simplicidade, ou seja, existem exatamente d arrestas encontrando cada vértice;

- racionalidade, ou seja, para cada vértice $v$ de $\Delta$ as arrestas adjancentes a v são da forma $v+t u_{i}, t \geq 0$, onde $u_{i} \in \mathbb{Z}^{n}$;

- suavidade, ou seja, para cada vértice $v$ de $\Delta$, os $u_{i}$ 's correspondentes podem ser escolhidos de modo a $\left\{u_{1}, \ldots, u_{d}\right\}$ ser uma $\mathbb{Z}$-base de $\mathbb{Z}^{n}$;

Seja $T$ um grupo de Lie abeliano de dimensão $n$ com $\mathfrak{t}=\operatorname{Lie}(T)$. Dado $\Delta \subset \mathfrak{t}^{*}$ politopo de Delzant, fixamos uma enumeração de suas facetas ( $(n-$ 1)-faces) $\left\{F_{1}^{(n-1)}, \ldots, F_{d}^{(n-1)}\right\}$. Consequentemente existirão $\left\{u_{1}, \ldots, u_{d}\right\} \subset \mathfrak{t}$, correspondendo biunivocamente as facetas de $\Delta$, e $\lambda_{1}, \ldots, \lambda_{d} \in \mathbb{R}$ de modo que:

$$
\Delta=\left\{\nu \in \mathfrak{t}^{*}:\left\langle\nu, u_{k}\right\rangle+\lambda_{k} \geqslant 0, \quad \forall k=1,2, \ldots, d\right\}
$$

Para cada face $F$ de $\Delta$ seja $S_{F} \subset\{1, \ldots, d\}$ o conjunto de índices $j$ tais que $F \subset F_{j}^{(n-1)}$. Desta forma podemos associar o cone:

$$
\text { cone }\left\{u_{s}: s \in S_{F}\right\}:=C_{F}:=\sum_{j \in S_{F}} \mathbb{R}_{\geq 0} u_{j}=\left\{\sum_{j \in S_{F}} a_{j} u_{j}: a_{j} \geq 0\right\}
$$

Observemos que $\Sigma:=\left\{C_{F}\right\}_{F \subset \Delta \text { face }}$ é cone dual a $\Delta$ em $\mathfrak{t}$. Notemos também que ordenando parcialmente as faces de $\Delta$ e os cones de $\Sigma$ pela inclusão, temos bijeção que reverte ordenação:

$$
\begin{aligned}
\{\text { faces de } \Delta\} & \stackrel{1-1}{\longmapsto}\{\text { cones de } \Sigma\} \\
F & \longmapsto \text { cone }\left\{u_{s}: s \in S_{F}\right\}=C_{F} \\
\Delta & \longmapsto\{0\}
\end{aligned}
$$

De fato, se $F^{\prime} \subset F$ são faces de $\Delta$, portanto $S_{F} \subset S_{F^{\prime}}$ e assim $C_{F} \subset C_{F^{\prime}}$. Em particular $F_{j}^{(n-1)} \mapsto \operatorname{cone}\left\{u_{j}\right\}$ e vértices $\mapsto$ cones n-dimensionais. Observemos 


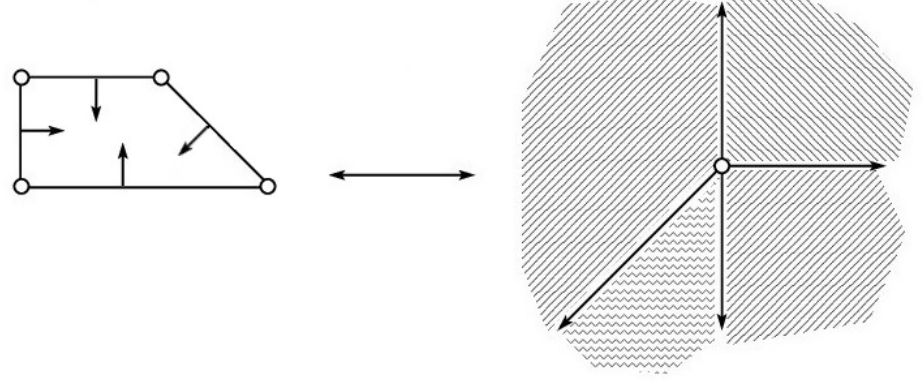

Figura 2.2: Polítopo e Fan

que com a escolha de um produto interno em $\mathrm{t}$, os elementos $\left\{u_{1}, \ldots, u_{d}\right\}$ podem ser considerados como vetores normais as facetas de $\Delta$ apontando para dentro via a identificação induzida de $t$ com $t^{*}$.

Seja $\Sigma=\Sigma_{\Delta}$ fan dual ao politopo $\Delta$. Analogamente as seções precendentes vamos construir variedade torica suave e compacta $X_{\Delta}=X(\Sigma)$ associada ao fan $\Sigma$.

Definimos a aplicação $\mathbb{R}$-linear $p: \mathbb{Z}^{d} \rightarrow \mathfrak{t}$ na qual $e_{j} \mapsto u_{j}$, para $j=1, \ldots, d$, e denotamos da mesma forma $p: \mathbb{C}^{d} \rightarrow \mathrm{t}_{\mathbb{C}}$ sua extensão complexa. A menos de renomeação dos vetores $u_{j}^{\prime} s$ a plicação $p$ é unicamente determinada pelo fan $\mathcal{F}$. Seja $\mathfrak{n} \subset \mathbb{R}^{d}$ o kernel de $p: \mathbb{R}^{d} \rightarrow \mathrm{t}$ e seja $\mathfrak{n}_{\mathbb{C}}=\operatorname{ker}\left(p: \mathbb{C}^{d} \rightarrow \mathfrak{t}_{\mathbb{C}}\right)$ sua complexificação. Além disso, associado a $\mathfrak{n}_{\mathbb{C}} \subset \mathbb{C}^{d}$ temos o subgrupo de Lie complexo $N_{\mathbb{C}} \subset \mathbb{T}_{\mathbb{C}}^{d}$.

Como um grupo de Lie complexo $N_{\mathbb{C}}$ é isomorfo a $\mathbb{T}_{\mathbb{C}}^{d-n}$. Se o cone gerado por $\left\{u_{1}, \ldots, u_{n}\right\}$ for n-dimencional em $\Sigma$

Para cada $S \subset\{1, \ldots, d\}$ seja:

$$
\begin{aligned}
\left(\mathbb{C}_{0}\right)^{S}: & =\left\{z=\left(z_{1}, \ldots, z_{d}\right) \in \mathbb{C}^{d}: z_{j}=0 \Longleftrightarrow j \notin S\right\} \\
& =\left\{z=\left(z_{1}, \ldots, z_{d}\right) \in \mathbb{C}^{d}: z_{j} \neq 0 \Longleftrightarrow j \in S\right\}
\end{aligned}
$$

Para $d=1$ as $\mathbb{T}$-órbitas serão a origem $\left(\mathbb{C}_{0}\right)^{\emptyset}=\{0\}$ e seu complemento $\left(\mathbb{C}_{0}\right)^{\{1\}}=\mathbb{C}^{*}$. Tomando-se "produto" fica claro que a associação $S \subset$ $\{1, \ldots, d\} \mapsto\left(\mathbb{C}_{0}\right)^{S} \subset \mathbb{C}^{d}$ é uma beijeção entre o conjuntos das partes de $\{1, \ldots, d\}$ e o conjuntos das $\mathbb{T}$-órbitas. Um fato importante a ser destacado é que o fecho de $\left(\mathbb{C}_{0}\right)^{S}$ é a união de todos $\left(\mathbb{C}_{0}\right)^{S^{\prime}}$ onde $S^{\prime} \subset S$.

$$
\mathcal{U}_{\Sigma}:=\bigcup_{S \in \mathcal{A}}\left(\mathbb{C}_{0}\right)^{S} \subset \mathbb{C}^{d}
$$

onde $\mathcal{A}:=\left\{S \subset\{1, \ldots, d\}: S^{c}=S_{F}\right.$ para alguma face $\mathrm{F}$ de $\left.\Delta\right\}$.

$\mathcal{A}:=\left\{S \subset\{1, \ldots, d\}:\right.$ cone $\left.\left\{u_{s}: u_{s} \notin S\right\} \in \Sigma\right\}$. 
Proposição 2.16 $\mathcal{U}_{\Sigma}$ é subconjunto aberto de $\mathbb{C}^{d}$ na qual $N_{\mathbb{C}}$ age livremente.

Proof. [7]

Corolário 2.17 Se $\mathcal{F}$ é o fan dual de $\Delta$, então $X_{\Sigma}:=\mathcal{U}_{\Sigma} / N_{\mathbb{C}}$ é variedade complexa suave com uma ação do toro complexo $T_{\mathbb{C}} \simeq \mathbb{T}_{\mathbb{C}}^{d} / N_{\mathbb{C}}$.

A partir de agora vamos construir atlas de $X(\Sigma)$ formado de cartas holomorficas associado aos vértices de $\Delta$.

Proposição 2.18 Dado $V \in \Delta$ vértice, associado a $V$ temos $C_{V}=\operatorname{cone}\left\{u_{s}\right.$ : $\left.s \in S_{V}\right\} \in \Sigma$ e seja $\mathcal{U}_{V}=\bigcup_{S}\left(\mathbb{C}_{0}\right)^{S}$, onde a união é sobre todos os $S \subset\{1, \ldots, d\}$ de modo que $S^{c} \subset S_{V}$, ou ainda, que cone $\left\{u_{s}: s \notin S\right\}$ seja face de $C_{V}$. Então:

1. $\mathcal{U}_{V} / N_{\mathbb{C}}$ é aberto em $X(\Sigma)$;

2. $\left\{\mathcal{U}_{V} / N_{\mathbb{C}}: C_{V}\right.$ é um cone $n$-dimensional de $\left.\Sigma\right\}$ é cobertura aberta de $X(\Sigma)$;

3. Para cada $V \in \Delta$ vértice existe $\phi_{V}: \mathcal{U}_{V} / N_{\mathbb{C}} \longrightarrow \mathbb{C}^{n}$ carta holomorfa;

Portanto:

$$
\left\{\left(\phi_{V}, \mathcal{U}_{V} / N_{\mathbb{C}}\right)\right\}_{V \in \Delta \text { vértice }}
$$

é atlas holomorfo de $X(\Sigma)$.

\section{Proof.}

1. Seja $C_{V}=\operatorname{cone}\left\{u_{s}: s \in S_{V}\right\}$ cone $n$-dimensional. Vamos mostrar que $\mathcal{U}_{\Sigma} \backslash \mathcal{U}_{V}$ é fechado. Notemos que

$$
\mathcal{U}_{\Sigma} \backslash \mathcal{U}_{V}=\bigcup_{S \in \mathcal{A}}\left(\mathbb{C}_{0}\right)^{S}
$$

onde $\mathcal{A}$ é a coleção de subconjuntos $S \subset\{1, \ldots, d\}$ tais que o cone $\left\{u_{s}\right.$ : $s \notin S\} \in \Sigma$, mas não é face do cone $C_{V}$. Assim, se $S^{c} \nsubseteq S_{V}$, então qualquer conjunto contendo $S^{c}$ também não vai estar contido em $S^{c}$, então o fecho de $\left(\mathbb{C}_{0}\right)^{S}$ está na união (2.1). Portanto $\mathcal{U}_{\Sigma} \backslash \mathcal{U}_{V}$ é a união finita de conjuntos fechados, consequentemente $\mathcal{U}_{V} / N_{\mathbb{C}}$ é aberto em $X(\Sigma)$.

2. Sendo $\Sigma$ fan completo para cada cone $n$-dimensional (fortemente convexo) existe ao menos um 1-cone que não o intersepta. Portanto os conjuntos $\mathcal{U}_{V}$, com $V$ vértices, formam uma cobertura de $\mathcal{U}_{\Sigma}$. 
3. Seja $C_{V} \in \Sigma$ cone $n$-dimensional associado ao vértice $V$. Por simplicidade vamos assumir $C_{V}=$ cone $\left\{u_{1}, \ldots, u_{n}\right\}$. Por definição temos $\mathcal{U}_{V}=\{z \in$ $\left.\mathbb{C}^{d}: z_{j} \neq 0, j=n+1, \ldots, d\right\}$. Seja $A^{V} \in \operatorname{Mat}(n \times d-n, \mathbb{R})$ dada por $u_{n+l}=\sum_{j=1}^{n} A_{j l} u_{j}=0$ para $l=1, \ldots, d-n$. Lembremos que a parametrização $N_{\mathbb{C}} \simeq \mathbb{T}_{\mathbb{C}}^{d-n}$ induz a ação:

$$
\zeta \cdot z:=\left(z_{1} \zeta_{1}^{A_{11}} \cdots \zeta_{d-n}^{A_{1-n}}, \ldots, z_{n} \zeta_{1}^{A_{n 1}} \cdots \zeta_{d-n}^{A_{n d-n}}, z_{n+1} \zeta_{1}, \ldots, z_{d} \zeta_{d-n}\right)
$$

Então a aplicação quociente $\mathcal{U}_{V} \longrightarrow \mathcal{U}_{V} / N_{\mathbb{C}}$ tem a seginte forma:

$$
\left(z_{1}, \ldots, z_{d}\right) \longmapsto\left[z_{1} z_{n+1}^{-A_{11}} \ldots z_{d}^{-A_{1 d-n}}: \cdots: z_{n} z_{n+1}^{-A_{d-n 1}} \ldots z_{d}^{-A_{d-n d-n}}: 1: \cdots: 1\right]
$$

A aplicação $\mathcal{U}_{V} / N_{\mathcal{C}} \longrightarrow \mathcal{U}_{V}$ dada por $\left[w_{1}: \cdots: w_{n}: 1: \cdots:\right.$ 1] $\mapsto\left(w_{1}, \cdots, w_{n}, 1, \cdots, 1\right)$ é claramente uma seção local trivializante do $N_{\mathbb{C}}$-fibrado $\mathcal{U}_{\Sigma} \longrightarrow X(\Sigma)$. Portanto, com a projeção adeguada temos carta local:

$$
\phi_{V}: \mathcal{U}_{V} / N_{\mathbb{C}} \longrightarrow \mathbb{C}^{n}
$$

Exemplo 2.19 Utilizando a construção precendente vamos construir atlas holomorfo associado ao seguinte politopo de Delzant:

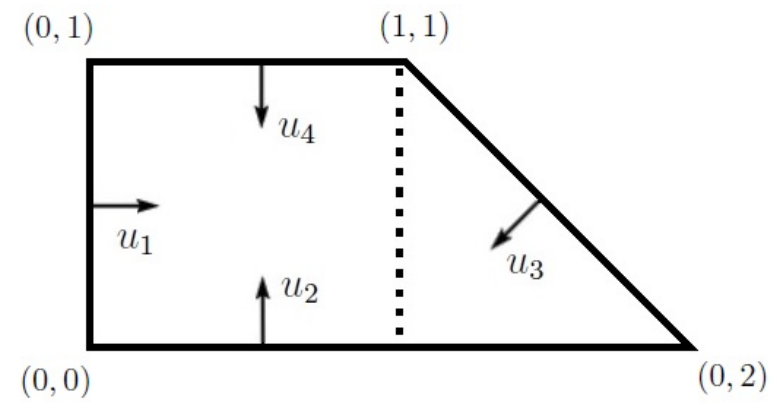

Figura 2.3: Polítopo de Delzant

Para cada vértice de $\Delta$ temos associado carta holomorfa:

$$
\begin{aligned}
\text { - } V_{0}= & (0,0) \in \Delta \rightsquigarrow S_{V_{0}}=\{1,2\} \rightsquigarrow C_{V_{0}}=\operatorname{cone}\left\{u_{1}, u_{2}\right\} \\
\mathcal{A}_{V_{0}}:= & \left\{S \subset\{1,2,3,4\}: S^{c} \subset S_{V_{0}}\right\}=\{\{3,4\},\{1,3,4\},\{2,3,4\},\{1,2,3,4\}\} \\
\mathcal{U}_{V_{0}}= & \bigcup_{S \in \mathcal{A}_{V_{0}}}\left(\mathbb{C}_{0}\right)^{S}=\left(\mathbb{C}_{0}\right)^{\{3,4\}} \cup\left(\mathbb{C}_{0}\right)^{\{1,3,4\}} \cup\left(\mathbb{C}_{0}\right)^{\{2,3,4\}} \cup\left(\mathbb{C}_{0}\right)^{\{1,2,3,4\}} \\
= & \{0\} \times\{0\} \times \mathbb{C}^{*} \times \mathbb{C}^{*} \cup \mathbb{C}^{*} \times\{0\} \times \mathbb{C}^{*} \times \mathbb{C}^{*} \cup \\
& \{0\} \times \mathbb{C}^{*} \times \mathbb{C}^{*} \times \mathbb{C}^{*} \cup \mathbb{C}^{*} \times \mathbb{C}^{*} \times \mathbb{C}^{*} \times \mathbb{C}^{*} \\
= & \mathbb{C} \times \mathbb{C} \times \mathbb{C}^{*} \times \mathbb{C}^{*}
\end{aligned}
$$




$$
A^{V_{0}} \in \operatorname{Mat}(2 \times 2, \mathbb{R}) \rightsquigarrow\left\{\begin{array}{l}
u_{3}+\left(1 . u_{1}+1 . u_{2}\right)=0 \\
u_{4}+\left(0 . u_{1}+1 . u_{2}\right)=0
\end{array} \quad \rightsquigarrow A^{V_{0}}=\left[\begin{array}{ll}
1 & 0 \\
1 & 1
\end{array}\right]\right.
$$

- Projeção: $\mathcal{U}_{V_{0}} \rightarrow \mathcal{U}_{V_{0}} / N_{\mathbb{C}} ;\left(z_{1}, z_{2}, z_{3}, z_{4}\right) \mapsto\left[z_{1} z_{3}^{-1}: z_{2} z_{3}^{-1} z_{4}^{-1}: 1: 1\right]$

- Seção: $\mathcal{U}_{V_{0}} / N_{\mathbb{C}} \rightarrow \mathcal{U}_{V_{0}}$ é dada por $\left[w_{1}: w_{2}: 1: 1\right] \mapsto\left(w_{1}, w_{2}, 1,1\right)$

- Carta $\phi_{V_{0}}: \mathcal{U}_{V_{0}} / N_{\mathbb{C}} \rightarrow \mathbb{C}^{2}$ é dada por

$$
\left[z_{1}: z_{2}: z_{3}: z_{4}\right] \longmapsto\left(z_{1} z_{3}^{-1}, z_{2} z_{3}^{-1} z_{4}^{-1}\right)
$$

- $V_{1}=(0,2) \in \Delta \rightsquigarrow S_{V_{1}}=\{2,3\} \rightsquigarrow C_{V_{1}}=\operatorname{cone}\left\{u_{2}, u_{3}\right\}$

$\mathcal{A}_{V_{1}}:=\left\{S \subset\{1,2,3,4\}: S^{c} \subset S_{V_{1}}\right\}=\{\{1,4\},\{1,2,4\},\{1,3,4\},\{1,2,3,4\}\}$

$$
\begin{aligned}
\mathcal{U}_{V_{1}}= & \bigcup_{S \in \mathcal{A}_{V_{1}}}\left(\mathbb{C}_{0}\right)^{S}=\left(\mathbb{C}_{0}\right)^{\{1,4\}} \cup\left(\mathbb{C}_{0}\right)^{\{1,2,4\}} \cup\left(\mathbb{C}_{0}\right)^{\{1,3,4\}} \cup\left(\mathbb{C}_{0}\right)^{\{1,2,3,4\}} \\
= & \mathbb{C}^{*} \times\{0\} \times\{0\} \times \mathbb{C}^{*} \cup \mathbb{C}^{*} \times \mathbb{C}^{*} \times\{0\} \times \mathbb{C}^{*} \cup \\
& \mathbb{C}^{*} \times\{0\} \times \mathbb{C}^{*} \times \mathbb{C}^{*} \cup \mathbb{C}^{*} \times \mathbb{C}^{*} \times \mathbb{C}^{*} \times \mathbb{C}^{*} \\
= & \mathbb{C}^{*} \times \mathbb{C} \times \mathbb{C} \times \mathbb{C}^{*}
\end{aligned}
$$

$$
A^{V_{1}} \in \operatorname{Mat}(2 \times 2, \mathbb{R}) \rightsquigarrow\left\{\begin{array}{l}
u_{1}+\left(1 . u_{2}+1 . u_{3}\right)=0 \\
u_{4}+\left(1 . u_{2}+0 . u_{3}\right)=0
\end{array} \quad \rightsquigarrow A^{V_{1}}=\left[\begin{array}{ll}
1 & 1 \\
1 & 0
\end{array}\right]\right.
$$

- Projeção: $\mathcal{U}_{V_{1}} \rightarrow \mathcal{U}_{V_{1}} / N_{\mathbb{C}} ;\left(z_{1}, z_{2}, z_{3}, z_{4}\right) \mapsto\left[1: z_{2} z_{1}^{-1} z_{4}^{-1}: z_{3} z_{1}^{-1}: 1\right]$

- Seção: $\mathcal{U}_{V_{0}} / N_{\mathbb{C}} \rightarrow \mathcal{U}_{V_{0}}$ é dada por $\left[1: w_{2}: w_{3}: 1\right] \mapsto\left(1, w_{2}, w_{3}, 1\right)$

- Carta $\phi_{V_{1}}: \mathcal{U}_{V_{1}} / N_{\mathbb{C}} \rightarrow \mathbb{C}^{2}$ é dada por

$$
\left[z_{1}: z_{2}: z_{3}: z_{4}\right] \longmapsto\left(z_{2} z_{1}^{-1} z_{4}^{-1}, z_{3} z_{1}^{-1}\right)
$$

- $V_{2}=(1,1) \in \Delta \rightsquigarrow S_{V_{2}}=\{3,4\} \rightsquigarrow C_{V_{2}}=\operatorname{cone}\left\{u_{3}, u_{4}\right\}$

$$
\begin{aligned}
\mathcal{A}_{V_{2}}:= & \left\{S \subset\{1,2,3,4\}: S^{c} \subset S_{V_{2}}\right\}=\{\{1,2\},\{1,2,3\},\{1,2,4\},\{1,2,3,4\}\} \\
\mathcal{U}_{V_{2}}= & \bigcup_{S \in \mathcal{A}_{V_{2}}}\left(\mathbb{C}_{0}\right)^{S}=\left(\mathbb{C}_{0}\right)^{\{1,2\}} \cup\left(\mathbb{C}_{0}\right)^{\{1,2,3\}} \cup\left(\mathbb{C}_{0}\right)^{\{1,2,4\}} \cup\left(\mathbb{C}_{0}\right)^{\{1,2,3,4\}} \\
= & \mathbb{C}^{*} \times \mathbb{C}^{*} \times\{0\} \times\{0\} \cup \mathbb{C}^{*} \times \mathbb{C}^{*} \times \mathbb{C}^{*} \times\{0\} \cup \\
& \mathbb{C}^{*} \times \mathbb{C}^{*} \times\{0\} \times \mathbb{C}^{*} \cup \mathbb{C}^{*} \times \mathbb{C}^{*} \times \mathbb{C}^{*} \times \mathbb{C}^{*} \\
= & \mathbb{C}^{*} \times \mathbb{C}^{*} \times \mathbb{C} \times \mathbb{C}
\end{aligned}
$$

$$
A^{V_{2}} \in \operatorname{Mat}(2 \times 2, \mathbb{R}) \rightsquigarrow\left\{\begin{array}{l}
u_{1}+\left(-1 . u_{3}+1 . u_{4}\right)=0 \\
u_{2}+\left(0 . u_{3}+1 . u_{4}\right)=0
\end{array} \quad \rightsquigarrow A^{V_{2}}=\left[\begin{array}{cc}
-1 & 0 \\
1 & 1
\end{array}\right]\right.
$$


- Projeção: $\mathcal{U}_{V_{2}} \rightarrow \mathcal{U}_{V_{2}} / N_{\mathbb{C}} ;\left(z_{1}, z_{2}, z_{3}, z_{4}\right) \mapsto\left[1: 1: z_{3} z_{1}: z_{4} z_{1}^{-1} z_{2}^{-1}\right]$

- Seção: $\mathcal{U}_{V_{2}} / N_{\mathbb{C}} \rightarrow \mathcal{U}_{V_{2}}$ é dada por $\left[1: 1: w_{3}: w_{4}\right] \mapsto\left(1,1, w_{3}, w_{4}\right)$

- Carta $\phi_{V_{2}}: \mathcal{U}_{V_{1}} / N_{\mathbb{C}} \rightarrow \mathbb{C}^{2}$ é dada por

$$
\left[z_{1}: z_{2}: z_{3}: z_{4}\right] \longmapsto\left(z_{3} z_{1}, z_{4} z_{1}^{-1} z_{2}^{-1}\right)
$$

- $V_{3}=(0,1) \in \Delta \rightsquigarrow S_{V_{3}}=\{1,4\} \rightsquigarrow C_{V_{3}}=\operatorname{cone}\left\{u_{1}, u_{4}\right\}$

$\mathcal{A}_{V_{3}}:=\left\{S \subset\{1,2,3,4\}: S^{c} \subset S_{V_{3}}\right\}=\{\{1,4\},\{1,2,4\},\{1,3,4\},\{1,2,3,4\}\}$

$$
\begin{aligned}
\mathcal{U}_{V_{3}}= & \bigcup_{S \in \mathcal{A}_{V_{3}}}\left(\mathbb{C}_{0}\right)^{S}=\left(\mathbb{C}_{0}\right)^{\{1,4\}} \cup\left(\mathbb{C}_{0}\right)^{\{1,2,4\}} \cup\left(\mathbb{C}_{0}\right)^{\{1,3,4\}} \cup\left(\mathbb{C}_{0}\right)^{\{1,2,3,4\}} \\
= & \{0\} \times \mathbb{C}^{*} \times \mathbb{C}^{*} \times\{0\} \cup \mathbb{C}^{*} \times \mathbb{C}^{*} \times \mathbb{C}^{*} \times\{0\} \cup \\
& \{0\} \times \mathbb{C}^{*} \times \mathbb{C}^{*} \times \mathbb{C}^{*} \cup \mathbb{C}^{*} \times \mathbb{C}^{*} \times \mathbb{C}^{*} \times \mathbb{C}^{*} \\
= & \mathbb{C} \times \mathbb{C}^{*} \times \mathbb{C}^{*} \times \mathbb{C}
\end{aligned}
$$

$$
A^{V_{3}} \in \operatorname{Mat}(2 \times 2, \mathbb{R}) \rightsquigarrow\left\{\begin{array}{l}
u_{2}+\left(0 . u_{3}+1 . u_{4}\right)=0 \\
u_{3}+\left(-1 . u_{3}+1 . u_{4}\right)=0
\end{array} \quad \rightsquigarrow A^{V_{2}}=\left[\begin{array}{cc}
0 & -1 \\
1 & 1
\end{array}\right]\right.
$$

- Projeção: $\mathcal{U}_{V_{3}} \rightarrow \mathcal{U}_{V_{3}} / N_{\mathbb{C}} ;\left(z_{1}, z_{2}, z_{3}, z_{4}\right) \mapsto\left[z_{1} z_{3}^{-1}: 1: 1: z_{4} z_{2} z_{3}^{-1}\right]$

- Seção: $\mathcal{U}_{V_{3}} / N_{\mathbb{C}} \rightarrow \mathcal{U}_{V_{3}}$ é dada por $\left[w_{1}: 1: 1: w_{4}\right] \mapsto\left(w_{1}, 1,1, w_{4}\right)$

- Carta $\phi_{V_{3}}: \mathcal{U}_{V_{3}} / N_{\mathbb{C}} \rightarrow \mathbb{C}^{2}$ é dada por

$$
\left[z_{1}: z_{2}: z_{3}: z_{4}\right] \longmapsto\left(z_{1} z_{3}^{-1}, z_{4} z_{2} z_{3}^{-1}\right)
$$




\section{3}

\section{Cohomologia de Poisson}

Neste capítulo vamos definir cohomologia de Poisson sobre uma variedade e linca-la com a cohomologia de DeRham. Definiremos estrutura Poisson-tórica e apresentaremos alguns exemplos. Ao final mostraremos alguns resultados sobre a não-exatitude de tais estruturas na cohomologia de Poisson. Considerações cohomológicas serão feitas sobre $H_{\Pi_{s t d}}^{*}\left((\mathbb{C})^{n}\right)$, onde $\Pi_{s t d}$ é estrutura quadrática canônica.

\section{1}

\section{Definição da Cohomologia de Poisson}

A cohomologia de Poisson foi introduzida por Lichnerowicz em [14], a peça fundamental para sua definição é o seguinte lema.

Lemma 3.1 Sejam $(M, \Pi)$ variedade Poisson e $A \in \mathfrak{X}^{k}(M)$ um $k$-campo de vetores. Então:

$$
[\Pi,[\Pi, A]]=0
$$

Proof. Por um lado, usando a identidade de Jacobi graduada do colchete de Schouten para $\Pi \in \mathfrak{X}^{2}(M)$ e $A \in \mathfrak{X}^{k}(M)$ temos:

$$
(-1)^{k-1}[\Pi,[\Pi, A]]-[\Pi,[A, \Pi]]+(-1)^{k-1}[A,[\Pi, \Pi]]=0
$$

por outro, usando a anticomutatividade graduada temos $[A, \Pi]=$ $-(-1)^{k-1}[\Pi, A]$ e, consequêntemente, $[\Pi,[\Pi, A]]=-\frac{1}{2}[A,[\Pi, \Pi]]$. Agora como $\Pi$ é estrutura poisson $[\Pi, \Pi]=0$, portanto, $[\Pi,[\Pi, A]]=0$.

Para cada bivetor $\Pi$ sobre uma variedade $M$ podemos definir um operador $\mathbb{R}$-linear $d_{\Pi}=d_{\Pi}^{*}: \mathfrak{X}^{*}(M) \longrightarrow \mathfrak{X}^{*+1}(M)$ sobre os espaços de multi-campos de $M$ da seguinte forma:

$$
d_{\Pi}(A):=d_{\Pi}^{k}(A):=[\Pi, A], \forall A \in \mathfrak{X}^{k}(M)
$$

Lema (3.1.1) nos garante que se $\Pi$ for Poisson $d_{\Pi}$ é um operador diferenciável no sentido que $d_{\Pi} \circ d_{\Pi}=0$ o que conrresponde a uma complexo de cocadeias $\left(\mathfrak{X}^{*}(M), d_{\Pi}\right)$ :

$$
\cdots \longrightarrow \mathfrak{X}^{k-1}(M) \stackrel{d_{\Pi}^{k-1}}{\longrightarrow} \mathfrak{X}^{k}(M) \stackrel{d_{\Pi}^{k}}{\longrightarrow} \mathfrak{X}^{k+1}(M) \longrightarrow \cdots
$$


chamado de complexo de Lichnerowicz. A cohomologia deste complexo é, por definição, a cohomologia de Poisson:

$$
H_{\Pi}^{k}(M)=\frac{\operatorname{ker}\left(d_{\Pi}^{k}: \mathfrak{X}^{k}(M) \longrightarrow \mathfrak{X}^{k+1}(M)\right)}{\operatorname{Im}\left(d_{\Pi}^{k-1}: \mathfrak{X}^{k-1}(M) \longrightarrow \mathfrak{X}^{k}(M)\right)}
$$

\section{2}

\section{Interpretação da Cohomologia de Poisson}

- 0-grupo de cohomologia $H_{\Pi}^{0}(M)$ :

O 0-grupo de cohomologia de Poisson $H_{\Pi}^{0}(M)$ é o grupo formado pelas funções $f \in C^{\infty}(M)$ de modo que $d_{\Pi}(f)=[\Pi, f]=-X_{f}=0$. Em outras palavras, $H_{\Pi}^{0}(M)$ é o espaço das funções Casimir de $\Pi$, ou seja, o espaço das integrais primeiras associadas a foliação simplética. Texto

- 1-grupo de Cohomologia $H_{\Pi}^{1}(M)$ :

O primeiro grupo de cohomologia de Poisson $H_{\Pi}^{1}(M)$ é o quociente do espaço dos campos de vetores Poisson, ou seja, que satisfazem $[\Pi, X]=0$, pelo espaço dos campos de vetores hamiltonianos, ou seja, dos campos $X \in \mathfrak{X}^{1}(M)$ da forma $X_{-f}=[\Pi, f]$. Os campos vetoriais Poisson são automorfismos infinitesimais das estruturas de Poisson, enquanto os campos vetoriais hamiltonianos podem ser interpretados como automorfismos infinitesimais internos. Assim, $H_{\Pi}^{1}(M)$ pode ser interpretado como o espaço de automorfismos infinitesimais externos de $\Pi$.

\section{- 2-grupo de Cohomologia $H_{\Pi}^{2}(M)$ :}

O segundo grupo de cohomologia de Poisson $H_{\Pi}^{2}(M)$ é o quociente do espaço dos bicampos $\Lambda$ que satisfazem $[\Pi, \Lambda]=0$ pelo espaço dos bicampos da forma $\Lambda=[\Pi, Y]$ para algum $Y \in \mathfrak{X}^{1}(M)$. Se $[\Pi, \Lambda]=0$ e $\varepsilon$ é um parâmetro (infinitesimal) formal, então $\Pi+\varepsilon \Lambda$ satisfaz a identidade Jacobi até os termos da ordem $\varepsilon^{2}$

$$
[\Pi+\varepsilon \Lambda, \Pi+\varepsilon \Lambda]=\varepsilon^{2}[\Lambda, \Lambda]=0 \bmod \varepsilon^{2}
$$

Portanto, pode-se ver $\Pi+\varepsilon \Lambda$ como uma deformação infinitesimal de $\Pi$ no espaço de tensores de Poisson. Por outro lado, até os termos da ordem $\varepsilon^{2}, \Pi+\varepsilon[\Pi, \Lambda]$ é igual a $\left(\phi_{Y}^{\varepsilon}\right)_{*} \Pi$, onde $\phi_{Y}^{\varepsilon}$ é o fluxo de $Y$ no tempo $\varepsilon$.

- 3-grupo de Cohomologia $H_{\Pi}^{3}(M)$ :

O terceiro grupo de cohomologia de Poisson $H_{\Pi}^{3}(M)$ pode ser interpretado como o espaço de obstruções à deformação formal. Suponha 
que tenhamos uma deformação infinitesimal $\Pi+\varepsilon \Lambda$, ou seja, $[\Pi, \Lambda]=0$. Então, a priori $\Pi+\varepsilon \Lambda$ satisfaz a identidade de Jacobi apenas em módulo $\varepsilon^{2}$.Para fazê-lo satisfazer a identidade de Jacobio módulo $\varepsilon^{3}$, temos que adicionar um termo $\varepsilon^{2} \Lambda_{2}$ tal que

$$
\left[\Pi+\varepsilon \Lambda+\varepsilon^{2} \Lambda_{2}, \Pi+\varepsilon \Lambda+\varepsilon^{2} \Lambda_{2}\right]=0 \bmod \varepsilon^{3}
$$

A equação a resolver é $2\left[\Pi, \Lambda_{2}\right]=-[\Lambda, \Lambda]$. Essa equação pode ser resolvida se, e somente se, a classe de cohomologia de $[\Lambda, \Lambda]$ em $H_{\Pi}^{3}(M)$ seja trivial. Similarmente, se (3.1) já estiver satisfeito

$$
\left[\Pi+\varepsilon \Lambda+\varepsilon^{2} \Lambda_{2}+\varepsilon^{3} \Lambda_{3}, \Pi+\varepsilon \Lambda+\varepsilon^{2} \Lambda_{2}+\varepsilon^{3} \Lambda_{3}\right]=0 \bmod \varepsilon^{4}
$$

temos que garantir que a classe de cohomologia de $\left[\Lambda, \Lambda_{2}\right]$ em $H_{\Pi}^{3}(M)$ se anule, e assim por diante.

O tensor Poisson $\Pi$ é um cociclo no complexo Lichnerowocz. Se a classe de cohomologia de $\Pi$ em $H_{\Pi}^{2}(M)$ é zero, ou seja, existe um campo de vetores $Y \in \mathfrak{X}^{1}(M)$ de modo que $[\Pi, Y]=\Pi$, então $\Pi$ é chamada estrutura de Poisson exata. Vamos agora a relação entre a cohomologia de De Rham e cohomologia Poisson.

\section{3}

\section{Cohomologia de Poisson vs Cohomologia de De Rham}

Sabemos que uma estrutura Poisson $\Pi$ sobre uma variedade $M$ induz um mapa entre fibrados:

$$
\Pi^{\#}: T^{*} M \longrightarrow T M
$$

na qual associa a cada covetor $\alpha \in T^{*} M$ um único vetor $\Pi^{\#}(\alpha) \in T M$ de modo que:

$$
\langle\alpha \wedge \beta, \Pi\rangle=\left\langle\beta, \Pi^{\#}(\alpha)\right\rangle, \forall \beta \in T^{*} M
$$

Este homomorfismo entre fibrados é um isomorfismo se, e somente se, П é não-degenerado, ou seja, simplético. Tomando-se potências exteriores da aplicação a cima podemos estende-la ao homomorfismo

$$
\Pi^{\#}: \Lambda^{k} T^{*} M \longrightarrow \Lambda^{k} T M
$$

e consequêntemente a uma aplicação $C^{\infty}(M)$-linear entre as seções:

$$
\Pi^{\#}: \Omega^{k}(M) \longrightarrow \mathfrak{X}^{k}(M)
$$


Esta última aplicação por sua vez traz a ligação entre as cohomologias de De Rham e Poisson no seguinte lema.

Lemma 3.2 Sejam $(M, \Pi)$ variedade Poisson e $\Pi^{\#}: \Omega^{*}(M) \longrightarrow \mathfrak{X}^{*}(M)$ mapa entre as seções. Então:

$$
\Pi^{\#}(\mathrm{~d} \eta)=-\left[\Pi, \Pi^{\#}(\eta)\right]=-d_{\Pi}\left(\Pi^{\#}(\eta)\right)
$$

Em outras palavras o seguinte diagrama é anticomutativo.

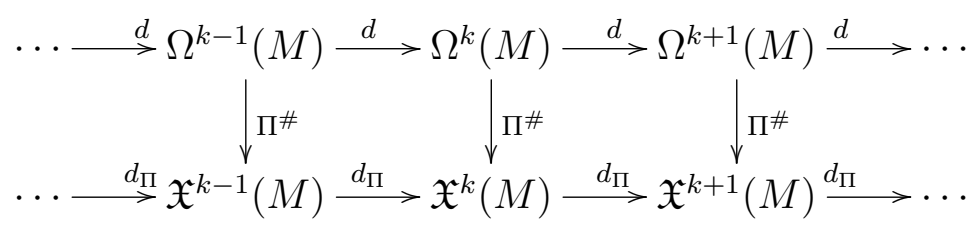

Proof. A prova se dá via indução sobre o grau de $\eta$ e usando a regra de Leibniz do colchete de Schouten.

Sem grandes dificuldades podemos perceber que lema (3.3.1) garante mapa entre entre as cohomologias:

$$
\Pi^{\#}: H_{D R}^{k}(M) \longrightarrow H_{\Pi}^{k}(M)
$$

Um observação importante a ser feita aqui é quando o bivetor Poisson $\Pi$ for não-degenerado, ou seja, quando $\Pi^{\#}: T^{*} M \longrightarrow T M$ for isomorfismo, nesse caso fica evidente pelo diagrama que $H_{\Pi}^{k}(M) \simeq H_{D R}^{k}(M)$.

Novamente, como mencionado na introdução, em geral o cálculo da cohomologia de Poisson para estruturas não-simpléticas é algo díficil demais e em aberto para a maioria das estruturas, tal dificuldade é resultado da carência de técnicas gerais como existem para a cohomologia de De Rham. Um dos teoremas provados nessa tese é exatamente o cálculo da cohomologia top de $\mathbb{C}^{n}$ para a estrutura quadrática canônica.

\section{4}

\section{Não-exatidão da estrutura Poisson-tórica}

Definição 3.1 Seja $\mathbb{T}_{\mathbb{C}}$ toro algébrico complexo e seja $X$ variedade tóric compacta e suave para ação $\rho: \mathbb{T}_{\mathbb{C}} \curvearrowright X$. Dizemos que um bivetor Poisson

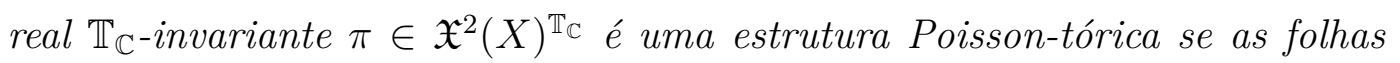

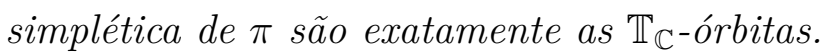

Lemma 3.3 Sejam $\Delta \subset \mathbb{R}^{n}$ politopo de Delzant, $\mathcal{F}$ fan dual a $\Delta e$ $X=X(\Sigma)=X_{\Delta}$ variedade tórica diferenciável associada. Então $X$ possui subvariedade tórica $Z$ equivariante a $\mathbb{C P}^{1}$. 
Proof. Seja $A$ uma aresta de $\Delta$ com $V_{1}$ e $V_{2}$ vértices adjacentes a $A$. A cada vértice está associada uma carta $\phi_{V_{j}}$ e um aberto coordenado $\mathcal{U}_{j} \subset$ $X(\Sigma)$ difeoformo a $\mathbb{C}^{n}$. A identificação induzida pela aresta $A$ nos abertos coordenados induz identificação em $\mathbb{C}^{n} \sqcup \mathbb{C}^{n}$ resultando num $\mathbb{C P}^{1}$.

Teorema 3.2 Sejam $\Delta \subset \mathbb{R}^{n}$ politopo de Delzant, $\mathcal{F}$ fan dual a $\Delta$ e $X=$ $X_{\Delta}$ variedade tórica diferenciável associada. Seja $\Pi \in \mathcal{X}^{2}(X)^{T_{\mathbb{C}}}$ estrutura Poisson T-invariante cujas folhas são exatamente as T-órbitas, ou seja, П é Poisson-tórica. Então $[\Pi] \neq 0$ em $H^{2}(X, \Pi)$, ou seja, П não é exata na cohomologia de Poisson por ela induzida.

\section{Proof.}

Suponhamos $[\Pi]=0$ em $H^{2}(X, \Pi)$, ou seja, que exista campo de vetores $Y \in \mathfrak{X}^{1}(X)$ tal que $d_{\Pi}(Y)=[\Pi, Y]=\Pi$. Desta forma, temos $\mathcal{L}_{Y}(\Pi)=[Y, \Pi]=$ $-\Pi$. Por $\mathcal{F}$ ser completo, temos $X$ é variedade compacta e fluxo $\phi^{Y}$ é completo, logo:

$$
\begin{aligned}
& \gamma(s):=\left(\phi_{-s}^{Y}\right)_{*} \Pi \in \mathfrak{X}^{1}(X), \forall s \in \mathbb{R} \\
& \gamma^{\prime}(s)=\left.\frac{d}{d t}\right|_{t=s}\left(\phi_{-s}^{Y}\right)_{*} \Pi=-\left(\phi_{-s}^{Y}\right)_{*} \Pi=-\gamma(s)
\end{aligned}
$$

o que chegamos a $\gamma^{\prime}(s)=-\gamma(s)$, ou ainda, $\gamma(s)=e^{-s} \gamma(0)=e^{-s} \Pi$. Trocado $s$ por $-s$ chegamos então:

$$
\left(\phi_{s}^{Y}\right)_{*} \Pi=e^{s} \Pi, \forall s \in \mathbb{R}
$$

o que, em particular, mostra que o posto de $\Pi$ é constante ao longo do fluxo de $Y$. Se considerarmos decomposição de $X$ via posto de $\Pi$ vemos que o fluxo está numa componente dessa decomposição, mas tal componente é união finita de folhas, logo $Y$ é tangente a elas e consequentemente tangente as folhas de

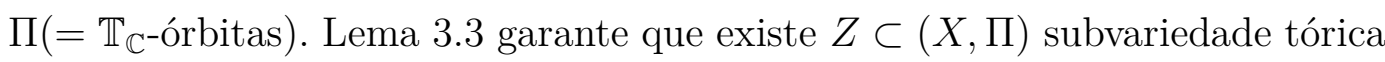

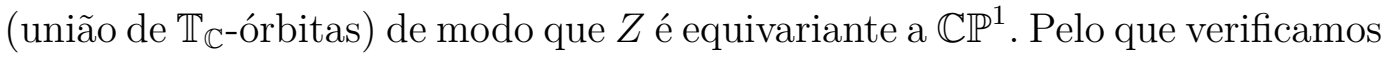
inicialmente $\left(Z,\left.\Pi\right|_{Z}\right)$ é subvariedade tórica de $X$.

$$
\left\{\left.\begin{array}{l}
\left.Y\right|_{Z} \in \mathfrak{X}^{2}(Z) \\
\left.\Pi\right|_{Z} \in \mathfrak{X}^{2}(Z)
\end{array} \rightsquigarrow d\right|_{Z}\left(\left.Y\right|_{Z}\right)=\left.\Pi\right|_{Z}\right.
$$

Isso significa que temos uma estrutura Poisson-tórica exata $\Pi^{\prime} \in \mathfrak{X}^{2}\left(\mathbb{C P}^{1}\right)$, ou seja, que existe $Y^{\prime} \in \mathfrak{X}^{1}\left(\mathbb{C P}^{1}\right)$ de modo que $\left[\Pi^{\prime}, Y^{\prime}\right]=\Pi^{\prime}$. Por invariancia, temos que $\Pi^{\prime}=\lambda \Pi_{s t d}, \lambda \in \mathbb{R}$, mas Arlo Caine provou que $\left[\Pi_{s t d}\right] \neq 0 \mathrm{em}$ $H_{\Pi_{s t d}}^{2}\left(\mathbb{C P}^{1}\right)$. Portanto $[\Pi] \neq 0$ em $H^{2}(X, \Pi)$. 


\section{5}

\section{Cohomologia Poisson e Cohomologia Invariante em $\mathbb{C}$}

\subsection{1}

\section{Da cohomologia para cohomologia invariante}

Seja $\mathbb{C}$ dotado da estrutura quadrática canônica $\Pi=-2 i z \bar{z} \partial_{z} \wedge \partial_{\bar{z}}$. Nakanishi em [8] calculou as cohomologias:

$$
H_{\Pi}^{0}(\mathbb{C}) \simeq \mathbb{R}, H_{\Pi}^{1}(\mathbb{C}) \simeq \mathbb{R}^{2}, H_{\Pi}^{2}(\mathbb{C}) \simeq \mathbb{R}
$$

utilizando fatoração polinomial e séries de potência. Nessa tese, usando uma estratégia alternativa, vamos provar o seguinte teorema.

Teorema 3.3 A cohomologia top $H_{\pi}^{4}\left(\mathbb{C}^{2}\right)$ é isomorfo a $\mathbb{R}^{4}$.

O mais relevante aqui é a maneira como vamos computar tal cohomologia de Poisson, no caso utilizando-se da cohomologia invariante. Genericamente, sabemos que se $\rho: G \curvearrowright M$ for ação de um grupo $G$ sobre uma variedade $M$ a comutatividade entre a diferencial de formas com o pullback, em particular $\rho_{g}^{*}(d f)=d\left(\rho_{g}^{*}(f)\right), \forall g \in G$, garante a passagem a um subcomplexo das formas invariantes:

$$
\cdots \longrightarrow \Omega^{k-1}(M)^{G} \longrightarrow \Omega^{k}(M)^{G} \longrightarrow \Omega^{k+1}(M)^{G} \longrightarrow \cdots
$$

Analogamente, $\Pi \in \mathfrak{X}^{2}(M)^{G}$ ser bivetor Poisson $\rho$-invariante garante a comutatividade entre a diferencial $d_{\Pi}$ e o pushforward de multicampos, pois:

$$
\begin{aligned}
d_{\pi}\left(\left(\rho_{g}\right)_{*} X\right) & =\left[\pi,\left(\rho_{g}\right)_{*} X\right]=\left[\left(\rho_{g}\right)_{*} \pi,\left(\rho_{g}\right)_{*} X\right] \\
& =\left(\rho_{g}\right)_{*}[\pi, X]=\left(\rho_{g}\right)_{*} d_{\pi}(X)
\end{aligned}
$$

Restringindo o complexo de multicampos aos multicampos invarienates essa comutatividade nos possibilita passagem ao subcomplexo de multicampos $\rho$-invariantes e, consequentemente, a cohomologia de Poisson invariante:

$$
\begin{gathered}
\cdots \longrightarrow \mathfrak{X}^{k-1}(M)^{G} \longrightarrow \mathfrak{X}^{k}(M)^{G} \longrightarrow \mathfrak{X}^{k+1}(M)^{G} \longrightarrow \cdots \\
H_{\Pi}^{k}(M)^{G}
\end{gathered}
$$

Vamos decompor teorema (3.5.1) em dois passos:

1. Qualquer $P \in \mathfrak{X}^{2}\left(\mathbb{C}^{2}\right)$ é Poisson cohomologo a um $Q \in \mathfrak{X}^{2}\left(\mathbb{C}^{2}\right)^{\mathbb{T}^{2}}$;

2. $H_{\Pi}^{4}\left(\mathbb{C}^{2}\right)^{\mathbb{T}^{2}}$ é isomorfo a $\mathbb{R}^{4}$; 
Proposição 3.4 Seja $M$ variedade munida de uma ação $\rho: \mathbb{S}^{1} \curvearrowright M$ juntamente com um bivetor Poisson $S^{1}$-invariante $\Pi \in \mathfrak{X}^{2}(M)^{\mathbb{S}^{1}}$ na qual é invertivel sobre um aberto denso $S^{1}$-invariante $U \subset M$. Sejam $\sigma$ o inverso simplético sobre $U$ e $\gamma:=\iota_{V} \sigma \in \Omega^{1}(M)$, onde $V$ é campo infinitesimal da ação. Se $P \in \mathfrak{X}^{k}(M)$ é $d_{\Pi}$-fechado de modo que $\iota_{\gamma}\left(\left.P\right|_{U}\right) \in \mathfrak{X}^{k-1}(M)$ se estende a um multicampo diferenciável em todo $M$, então $P$ é Poisson cohomologo a um multicampo $Q \in \mathfrak{X}^{k}(M)^{\mathbb{S}^{1}}$.

Proof. A filosofia desta prova basea-se na construção de um operador homotópico no complexo de multicampos, essa construção é fortemente motivada pela teoria de DeRham invariante. Vamos ao caso de formas primeiro. Seja $\alpha \in \Omega^{k}(U)$, considere sua média:

$$
\operatorname{Av}(\alpha)=\frac{1}{2 \pi} \int_{0}^{2 \pi} \psi_{t}^{*} \alpha d t \in \Omega^{k}(U)^{\mathbb{S}^{1}}
$$

onde $\psi_{t}$ é o fluxo de $V$. Definindo caminho de formas $\delta(t):=\alpha-\psi_{t}^{*} \alpha$ notemos que:

$$
\alpha-\frac{1}{2 \pi} \int_{0}^{2 \pi} \psi_{t}^{*} \alpha d t=\frac{1}{2 \pi} \int_{0}^{2 \pi} \delta(t) d t
$$

Evidentemente esse caminho se anula em $t=0$ e pelo teorema fundamental do cálculo vale que:

$$
\delta(t)=\int_{0}^{t} \delta^{\prime}(s) d s
$$

pela fórmula de Cartan

$$
\begin{aligned}
\delta^{\prime}(s) & =\frac{d}{d s} \psi_{s}^{*} \alpha=\psi_{s}^{*}\left(-\mathcal{L}_{V} \alpha\right)=\psi_{s}^{*}\left(-d \iota_{V} \alpha-\iota_{V} d \alpha\right) \\
& =d\left(-\psi_{s}^{*} \circ \iota_{V}\right) \alpha+\left(-\psi_{s}^{*} \circ \iota_{V}\right) d \alpha
\end{aligned}
$$

Pelo fato que a integração comuta com a diferencial exterior obtemos a igualdade:

$$
\begin{aligned}
\alpha-A v(\alpha) & =\frac{1}{2 \pi} \int_{0}^{2 \pi} \int_{0}^{t}\left(d\left(-\psi_{s}^{*} \circ \iota_{V}\right) \alpha+\left(-\psi_{s}^{*} \circ \iota_{V}\right) d \alpha\right) d s d t \\
& =\left(d \circ \frac{1}{2 \pi} \int_{0}^{2 \pi} \int_{0}^{t}\left(-\psi_{s}^{*} \circ \iota_{V}\right) d s d t\right) \alpha+\left(\frac{1}{2 \pi} \int_{0}^{2 \pi} \int_{0}^{t}-\left(\psi_{s}^{*} \circ \iota_{V}\right) d s d t \circ d\right) \alpha
\end{aligned}
$$

na qual obtemos uma homotopia de cadeia entre o operador identidade e o operador média

$$
\mathrm{I}-\mathrm{Av}=d K+K d, \quad K=\left(\frac{1}{2 \pi} \int_{0}^{2 \pi} d t\right) \circ\left(\int_{0}^{t} d s\right) \circ\left(-\psi_{s}^{*} \circ \iota_{V}\right)
$$

O que fazemos agora é transferir o operador $K: \Omega^{\bullet}(U) \longrightarrow$ 
$\Omega^{\bullet-1}(U)$ para o complexo de Poisson $(U, \Pi)$. Para isso nos utilizaremos do $C^{\infty}(U)$-isomorfismo $\Pi^{\#}: \Omega^{\bullet}(U) \longrightarrow \mathfrak{X}^{\bullet}(U)$, cuja inversa denotaremos por $\sigma^{\#}$. Essa transferência se dá via a comutatividade do seguinte diagrama:

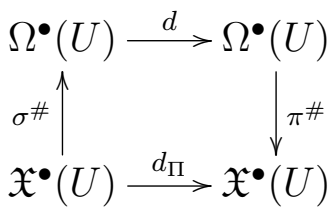

Através desse diagrama portanto podemos reescrever (3.2) ao nível de multicampos:

$$
\begin{aligned}
\mathrm{I}-\left(\pi^{\#} \circ \mathrm{Av} \circ \sigma^{\#}\right) & =\pi^{\#} \circ d K \circ \sigma^{\#}+\pi^{\#} \circ K d \circ \sigma^{\#}= \\
& =d_{\pi} \circ\left(\pi^{\#} \circ K \circ \sigma^{\#}\right)++\left(\pi^{\#} \circ K \circ \sigma^{\#}\right) \circ d_{\pi}
\end{aligned}
$$

Para finalizar a prova precisamos garantir que os operadores acima, definidos em $\mathfrak{X}^{\bullet}(U)$, sejam restrições de operadores em $\mathfrak{X}^{\bullet}(M)$.

Para o caso de $\pi^{\#} \circ \mathrm{Av} \circ \sigma^{\#}$, vamos verificar que é a restrição a menos de sinal do operador média para multicampos $A v: \mathfrak{X}^{\bullet}(M) \longrightarrow \mathfrak{X}^{\bullet}(M)^{\mathbb{S}^{1}}$ :

$$
\operatorname{Av}(P)=\frac{1}{2 \pi} \int_{0}^{2 \pi} \psi_{t *} P d t=-\frac{1}{2 \pi} \int_{0}^{2 \pi} \psi_{-t *} P d t
$$

Para isso precisamos fazer duas observações: o primeiro é que os isomorfismos de fibrados $\pi^{\#}$ e $\sigma^{\#}$ comutam com a integração. O segundo é que tanto $\pi^{\#}$ como $\sigma^{\#}$ são $\mathbb{T}^{2}$-invariantes, logo:

$\sigma\left(\psi_{t}^{*} \cdot, \psi_{t}^{*}\right)=\sigma(\cdot, \cdot) \Leftrightarrow \sigma\left(\cdot, \psi_{t}^{*} \cdot\right)=\sigma\left(\psi_{-t}^{*} \cdot \cdot\right) \Leftrightarrow \psi_{t}^{*} \circ \sigma^{\#}=\sigma^{\#} \circ \psi_{-t}^{*} \Leftrightarrow \pi^{\#} \circ \psi_{t}^{*} \circ \sigma^{\#}=\psi_{-t}^{*}$

portanto

$$
\pi^{\#} \circ \mathrm{Av} \circ \sigma^{\#}=\pi^{\#} \circ\left(\frac{1}{2 \pi} \int_{0}^{2 \pi} \psi_{t}^{*} d t\right) \circ \sigma^{\#}=\frac{1}{2 \pi} \int_{0}^{2 \pi} \pi^{\#} \circ \psi_{t}^{*} \circ \sigma^{\#} d t=-\mathrm{Av}
$$

Precisamos agora analizar o outro operador $\pi^{\#} \circ K \circ \sigma^{\#}$. Espandindo $K$ obtemos

$\pi^{\#} \circ K \circ \sigma^{\#}=\left(\pi^{\#} \circ\left(\frac{1}{2 \pi} \int_{0}^{2 \pi} d t\right) \circ \sigma^{\#}\right) \circ\left(\pi^{\#} \circ\left(\int_{0}^{t} d s\right) \circ \sigma^{\#}\right) \circ\left(\pi^{\#} \circ\left(-\phi_{s}^{*} \circ \iota_{V}\right) \circ \sigma^{\#}\right)$

usando novamente a comutatividade entre a integração e o isomorfismo de fibrados obtemos que os dois primeiros operadores são exatamente:

$$
\frac{1}{2 \pi} \int_{0}^{2 \pi} d t, \quad \int_{0}^{t} d s
$$


operadores de integração definidos espaço de caminhos de multicampos parametrizados por $[0,2 \pi]$ e $[0, t]$, respectivamente. Claramente são operadores definidos em todo $\mathfrak{X}^{\bullet}(M)$.

O terceiro operador

$$
\pi^{\#} \circ\left(-\psi_{s}^{*} \circ \iota_{V}\right) \circ \sigma^{\#}=-\psi_{-s}^{*} \circ\left(\pi^{\#} \circ \iota_{V} \circ \sigma^{\#}\right)=-\psi_{-s}^{*} \circ \iota_{\sigma}(V)
$$

Notemos que $\sigma^{\#}(V)$ está definido somente em $U$, mas por hipótese $\iota_{\sigma}(V)(P)=$ $\iota_{\gamma}(P)$ se estende a todo $M$. Além disso, por $U$ ser denso e $P$ ser $d_{\Pi}$-fechado, existe $Q \in \mathfrak{X}^{k-1}(M)$ de modo que:

$$
P-(-A v(P))=d_{\Pi}(Q), \quad A v(P) \in \mathfrak{X}^{k}(M)^{\mathbb{S}^{1}}
$$

Lema da divisão ou ainda lema de Hadamard para funções diferenciáveis em $\mathbb{R}^{n}$ diz que o ideal das funções que se anulam na origem é gerado pelas funções coordenadas:

$$
f(x)=x_{1} g_{1}(x)+\cdots+x_{n} g_{n}(x), g_{i}(x) \in C^{\infty}\left(\mathbb{R}^{n}\right)
$$

A prova é essencialemente aplicar o teorema fundamental do cálculo para o segmento de reta ligado a origem ao ponto genêrico. Os fatores $g_{i}$ 's na decomposição são o resultado da aplicação de operadores integrais e diferenciais que dependem suavemente de parâmetros diferenciáveis. Precisamos de uma versão do lema de divisão onde a origem é substituída por uma família apropriada de subespaços através da origem. A dependência diferênciável dos parâmentros é a peça chave da demonstração.

Lemma 3.4 Seja $f(z, w) \in C^{\infty}\left(\mathbb{C}^{2}\right)$ tal que se anula na união dos dois eixos complexos. Identificando $\mathbb{C}^{2} \simeq \mathbb{R}^{4}$ via $z=(x, y)$ e $w=(u, v)$, então $f$ pertence ao ideal gerado por $\{x u, x v, y u, y v\}$.

Proof. Seja $f(z, w)$ como no enunciado. Fixamos $\left(x_{0}, y_{0}, 0,0\right)$ sobre o eixo- $z$. Consideremos $p(u, v) \in C^{\infty}(\mathbb{C})$ dada por

$$
p(u, v):=f\left(x_{0}, y_{0}, u, v\right),(u, v) \in \mathbb{R}^{2}
$$

Por hipótese, $p(0,0)=0$ e, desta forma, aplicando lema da divisão sobre $p$ obtemos:

$$
p(u, v)=u q_{1}(u, v)+v q_{2}(u, v), \quad q_{1}, q_{2} \in C^{\infty}(\mathbb{C}) .
$$

Como mencionado anteriormente o lema da divisão depende suavemente dos parâmentros, no caso de $x_{0}$ e $y_{0}$. Isso significa que obtemos a seguinte 
decomposição:

$$
f=u g_{1}+v g_{2}, \quad g_{1}, g_{2} \in C^{\infty}\left(\mathbb{C}^{2}\right)
$$

Afirmamos que $g_{1}(0,0, u, v)=g_{2}(0,0, u, v)=0$. O motivo dessa relação está no que $g_{1}(0,0, u, v)$ e $g_{2}(0,0, u, v)$ dependerem do valor $\mathrm{f}$ ao longo do segmento de reta ligando a origem ao ponto $(0,0, u, v)$. Aplicando novamente lema da divisão a cada $g_{i}\left(x, y, u_{0}, v_{0}\right)$ resulta

$$
f=u g_{1}+v g_{2}=u\left(x h_{1}+y h_{2}\right)+v\left(x h_{3}+y h_{4}\right)=u x h_{1}+u y h_{2}+v x h_{3}+v y h_{4}
$$

onde $h_{i} \in C^{\infty}\left(\mathbb{C}^{2}\right)$, o que prova o lema.

Teorema 3.5 A inclusão do complexo Poisson invariante no complexo Poisson

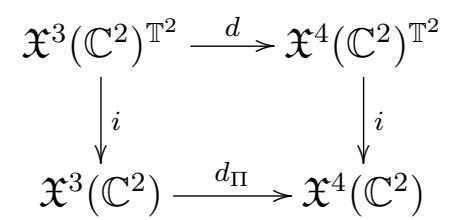

induz um isomorfismo $i: H_{\Pi}^{4}\left(\mathbb{C}^{2}\right)^{\mathbb{T}^{2}} \simeq H_{\Pi}^{4}\left(\mathbb{C}^{2}\right)$.

Proof. Primeiramente, vamos verificar que os operadores $d_{\Pi}$ e $A v, A v(X):=$ $-\frac{1}{2 \pi} \int_{0}^{2 \pi} \psi_{-t *} X d t \operatorname{com} X \in \mathfrak{X}^{\bullet}\left(\mathbb{C}^{2}\right)$, comutam.

$$
\begin{aligned}
\left(A v \circ d_{\Pi}\right)(X) & \left.=A v([\Pi, X])=-\frac{1}{2 \pi} \int_{0}^{2 \pi}\left(\psi_{-t}\right)_{*}([\Pi, X]) d t=-\frac{1}{2 \pi} \int_{0}^{2 \pi}\left[\Pi,\left(\psi_{-t}\right)_{*} X\right)\right] d t= \\
& =\left[\Pi,-\frac{1}{2 \pi} \int_{0}^{2 \pi}\left(\psi_{-t}\right)_{*} X d t\right]=\left(d_{\Pi} \circ A v\right)(X)
\end{aligned}
$$

onde mais uma vez usamos a $\mathbb{T}^{2}$-invariância de $\Pi$. Verificada a comutatividade, vamos então provar que $i: H_{\Pi}^{4}\left(\mathbb{C}^{2}\right)^{\mathbb{T}^{2}} \longrightarrow H_{\Pi}^{4}\left(\mathbb{C}^{2}\right)$ é injetiva. Dado $P \in$ $\mathfrak{X}^{4}\left(\mathbb{C}^{2}\right)^{\mathbb{T}^{2}}$ de modo que $[P]=0$ em $H_{\Pi}^{4}\left(\mathbb{C}^{2}\right)$, logo existe $Q \in \mathfrak{X}^{3}\left(\mathbb{C}^{2}\right)$ tal que $P=d_{\Pi}(Q)$. Pelo que verificamos inicialmente:

$$
P=A v(P)=A v\left(d_{\Pi}(Q)\right)=d_{\Pi}(A v(Q))
$$

o que mostra que $[P]=0$ em $H_{\Pi}^{4}\left(\mathbb{C}^{2}\right)^{\mathbb{T}^{2}}$.

Agora, vamos a parte mais trabalhosa da prova, $i$ é sobrejetiva. Precisamos mostrar que se $P \in \mathfrak{X}^{4}\left(\mathbb{C}^{2}\right)$, então existem $Q \in \mathfrak{X}^{4}\left(\mathbb{C}^{2}\right)^{\mathbb{T}^{2}}$ e $R \in \mathfrak{X}^{3}\left(\mathbb{C}^{2}\right)$ tais que:

$$
P=Q+d_{\Pi}(R)
$$


o que em palavras diz que qualquer elemento de $\mathfrak{X}^{4}\left(\mathbb{C}^{2}\right)$ é Poisson cohomólogo com algum elemento de $\mathfrak{X}^{4}\left(\mathbb{C}^{2}\right)^{\mathbb{T}^{2}}$. Seja $P=p^{\partial} / \partial x \wedge \partial / \partial y \wedge \partial / \partial u \wedge \partial / \partial v \in \mathfrak{X}^{4}\left(\mathbb{C}^{2}\right)$. Para a notação não ficar sobrecarreagada vamos escrever os multivetores com sub-indices, $\log O=p \partial_{x, y, u, v}$. Definimos as funções e os multicampos:

$$
\begin{gathered}
p_{0}:=p(0,0,0,0), p_{1}:=p(x, y, 0,0)-p_{0}, p_{2}:=p(0,0, u, v)-p_{0}, p_{3}:=p-p_{0}-p_{1}-p_{2} \\
P_{j}:=p_{j} \partial_{x, y, u, z}, \quad j=0, \ldots, 3
\end{gathered}
$$

O teorema estará provado uma vez que verificarmos que cada $P_{j}, j=0, \ldots, 3$, for Poisson cohomólgo com multicampos invariantes. Observe que $p=p_{0}+$ $p_{1}+p_{2}+p_{3}$ e as 4 componentes $p_{j}$ 's possuem as seguintes propriedades:

- $p_{0}$ é constante;

- $p_{1}$ depende somente das variáveis $x$ e $y$ e se anula na origem;

- $p_{2}$ depende somente das variáveis $u$ e $v$ e se anula na origem;

- $p_{3}$ se anula na união dos dois eixos complexos;

Pois bem, notemos que $\partial_{x, y, u, v} \in \mathfrak{X}^{4}\left(\mathbb{C}^{2}\right)^{\mathbb{T}^{2}}$ e que a função constante $p_{0}$ é $\mathbb{T}^{2}$-invariante, logo:

$$
P_{0}:=p_{0} \partial_{x, y, u, v} \in \mathfrak{X}^{4}\left(\mathbb{C}^{2}\right)^{\mathbb{T}^{2}}
$$

Para seguir a analise de cada $P_{j}$ precismoa expressar $d_{\Pi \text { }}$ sobre campos de trivetores:

$$
\begin{gathered}
d_{\Pi}: \mathfrak{X}^{3}\left(\mathbb{C}^{2}\right) \longrightarrow \mathfrak{X}^{4}\left(\mathbb{C}^{2}\right) \\
f \partial_{x, u, v}+g \partial_{y, u, v} \mapsto\left(\left(x^{2}+y^{2}\right)\left(\partial_{x} f+\partial_{y} g\right)-2 x f-2 y g\right) \partial_{x, y, u, v} \\
h \partial_{x, y, u}+l \partial_{x, y, v} \mapsto\left(\left(u^{2}+v^{2}\right)\left(\partial_{u} h+\partial_{v} l\right)-2 u h-2 v l\right) \partial_{x, y, u, v}
\end{gathered}
$$

Vamos modificar $P_{1}=p_{1} \partial_{x, y, u, v}$ por um cobordo apropriado. Aplicando lema da divisão para $p_{1}(x, y, u, v)$ (para as coordenadas $x, y$ ) obtemos:

$$
p_{1}=x m+y n, \quad m, n \in C^{\infty}(\mathbb{C})
$$

Seja $R_{1}:=\frac{1}{2} m \partial_{x, u, v}+\frac{1}{2} n \partial_{y, u, v}$. Então equação (3.5) implica que:

$$
\begin{aligned}
P_{1}+d_{\Pi}\left(R_{1}\right) & =\left(p_{1}-x m-y n+\frac{1}{2}\left(x^{2}+y^{2}\right)\left(\partial_{x} m+\partial_{y} n\right)\right)= \\
& =\frac{1}{2}\left(x^{2}+y^{2}\right)\left(\partial_{x} m+\partial_{y} n\right) \partial_{x, y, u, v}
\end{aligned}
$$

Queremos verificar se estamos nas hipóteses de aplicar o operador de média com relação à ação sobre o primeiro fator. O campo vetorial fundamental 
para esta ação é o campo vetorial rotacional:

$$
V_{1}=-y \partial_{x}+x \partial_{y}
$$

A forma simplética inversa de $\Pi$ em $\mathbb{C}^{2} \backslash\{(z, w): z w=0\}$ é:

$$
\sigma=-\frac{1}{x^{2}+y^{2}} d x \wedge d y-\frac{1}{u^{2}+v^{2}} d u \wedge d v
$$

e sua contração com o campo de vetor rotacional $V_{1}$ é:

$$
\iota_{V_{1}} \sigma=-\frac{1}{x^{2}+y^{2}}(x d x+y d y) \in \Omega^{1}\left(\mathbb{C}^{2} \backslash\{(z, w): z w=0\}\right)
$$

Portanto, os campos multivetores de grau 4 cuja contração com V1 se estende a um campo vetorial de grau suave em $\mathbb{C}^{2}$ é o submódulo

$$
\left(x^{2}+y^{2}\right) \mathfrak{X}^{4}\left(\mathbb{C}^{2}\right)
$$

Pela relação (3.6) temos $P_{1}+d_{\Pi}\left(R_{1}\right) \in\left(x^{2}+y^{2}\right) \mathfrak{X}^{2}\left(\mathbb{C}^{2}\right)$. Aplicando proposição (3.4), existe $T_{1} \in \mathfrak{X}^{3}\left(\mathbb{C}^{2}\right)$ de modo que

$$
P_{1}+d_{\Pi}\left(R_{1}\right)+d_{\Pi}\left(T_{1}\right)=P_{1}+d_{\Pi}\left(R_{1}+T_{1}\right)=\operatorname{Av}_{1}\left(p_{1}+d_{\Pi}\left(R_{1}\right)\right)
$$

onde $A v_{j}$ é o operador média com relação a ação $\mathbb{S}^{1}$ sobre o $j$ fator complexo. Nossa última observação é que o multicampo $P_{1}+d_{\Pi}\left(R_{1}\right)$ não depende das variáveis reais $u$ e $v$, portanto é invariante a ação de $\mathbb{S}^{1}$ sobre o segundo fator complexo. Logo

$$
P_{1}+d_{\pi}\left(R_{1}+T_{1}\right)=\operatorname{Av}_{1}\left(P_{1}+d_{\pi}\left(R_{1}\right)\right)=\operatorname{Av}\left(P_{1}+d_{\pi}\left(R_{1}\right)\right) \in \mathfrak{X}^{4}\left(\mathbb{C}^{2}\right)^{T}
$$

Portanto $P_{1}$ é Poisson cohomologo a um multicampo $\mathbb{T}^{2}$-invariante.

De forma inteiramente analoga analizamos $P_{2}=p_{2} \partial_{x, y, u, v}$ e produzimos $R_{2}+T_{2} \in \mathfrak{X}^{3}\left(\mathbb{C}^{2}\right)$ de modo a termos:

$$
P_{2}+d_{\pi}\left(R_{2}+T_{2}\right)=\operatorname{Av}_{2}\left(p_{2}+d_{\pi}\left(R_{2}\right)\right)=\operatorname{Av}\left(p_{2}+d_{\pi}\left(R_{2}\right)\right) \in \mathfrak{X}^{4}\left(\mathbb{C}^{2}\right)^{\mathbb{T}^{2}}
$$

Portanto, novamente, $P_{2}$ é Poisson cohomologo a um multicampo $\mathbb{T}^{2}$-invariante. Vamos agora analizar o último caso.

Para mostrar que $P_{3}=p_{3} \partial_{x, y, u, v}$ é Poisson cohomólo a um 4-vetor invariante, vamos fazer isso em duas etapas. Pelo lema (3.4) podemos escrever:

$$
p_{3}=x u l_{1}+x v l_{2}+y l_{3}+y v l_{4}=x\left(u l_{1}+v l_{2}\right)+y\left(u l_{3}+v l_{4}\right)
$$


Para o primeiro passo definimos:

$$
W_{1}=\frac{1}{2}\left(u l_{1}+v l_{2}\right) \partial_{x, u, v}+\frac{1}{2}\left(u l_{3}+v l_{4}\right) \partial_{y, u, v}
$$

por $(3.4)$

$$
P_{3}+d_{\pi}\left(W_{1}\right)=\frac{1}{2}\left(x^{2}+y^{2}\right)\left(u\left(\partial_{x} l_{1}+\partial_{y} l_{3}\right)+v\left(\partial_{x} l_{2}+v \partial_{y} l_{4}\right)\right) \partial_{x, y, u, v}
$$

O segundo passo é definir:

$W_{2}=\frac{1}{2} \kappa_{1} \partial_{x, u, v}+\frac{1}{2} \kappa_{2} \partial_{y, u, v}, \quad \kappa_{1}=\frac{1}{2}\left(x^{2}+y^{2}\right)\left(\partial_{x} l_{1}+\partial_{y} l_{3}\right), \kappa_{2}=\frac{1}{2}\left(x^{2}+y^{2}\right)\left(\partial_{x} l_{2}+v \partial_{y} l_{4}\right)$

e novamente por (3.4)

$$
P_{3}+d_{\pi}\left(W_{1}+W_{2}\right)=\frac{1}{4}\left(x^{2}+y^{2}\right)\left(u^{2}+v^{2}\right) l_{5} \partial_{x, y, u, v}, \quad l_{5} \in C^{\infty}\left(\mathbb{C}^{2}\right)
$$

A partir dessa última ralação podemos aplicar a $P_{3}+d_{\pi}\left(W_{1}+W_{2}\right)$ o operador de homotopia que o leva para sua média

$$
P_{3}+d_{\pi}\left(W_{1}+W_{2}+W_{3}\right)=\operatorname{Av}_{1}\left(P_{3}+d_{\pi}\left(W_{1}+W_{2}\right)\right), \quad W_{3} \in \mathfrak{X}^{3}\left(\mathbb{C}^{2}\right)
$$

Segue-se que:

$$
\operatorname{Av}_{1}\left(\left(x^{2}+y^{2}\right)\left(u^{2}+v^{2}\right) \mathfrak{X}^{4}\left(\mathbb{C}^{2}\right)\right) \subset\left(u^{2}+v^{2}\right) \mathfrak{X}^{4}\left(\mathbb{C}^{2}\right)
$$

então podemos aplicar a $\operatorname{Av}_{1}\left(P_{3}+d_{\pi}\left(W_{1}+W_{2}\right)\right)$ operador homotpia na qual leva a sua média:

$$
\begin{aligned}
P_{3}+d_{\pi}\left(W_{1}+W_{2}+W_{3}+W_{4}\right) & =\operatorname{Av}_{2} \circ \operatorname{Av}_{1}\left(P_{3}+d_{\pi}\left(W_{1}+W_{2}\right)\right)= \\
& =\operatorname{Av}\left(P_{3}+d_{\pi}\left(W_{1}+W_{2}\right)\right) \in \mathfrak{X}^{4}\left(\mathbb{C}^{2}\right)^{\mathbb{T}^{2}}
\end{aligned}
$$

o que mostra que $P_{3}$ é cohomólogo com elemento invariante.

\subsection{2}

\section{O complexo invariante e funções no quadrante positivo}

Os campos de multivetores em $\mathbb{C}^{2}$ são um módulo livre com respeito a uma base canônica associada às coordenadas fixas. Para ter certeza de que a redução do cálculo da cohomologia de Poisson ao complexo invariante é útil, deve-se mostrar que o complexo invariante simplifica o cálculo da cohomologia 
de Poisson. Esse fato é consequência de duas propriedades:

- Os campos de multivetores invariantes são um módulo livre sobre as funções invariantes (com o referêncial canônico);

- As funções invariantes são os pull back das funções no quadrante positivo de $\mathbb{R}^{2}$, e essa identificação simplifica o cálculo explícito da cohomologia invariante.

Lemma 3.5 Ambos $\mathfrak{X}^{3}\left(\mathbb{C}^{2}\right)^{\mathbb{T}^{2}}$ e $\mathfrak{X}^{4}\left(\mathbb{C}^{2}\right)^{\mathbb{T}^{2}}$ são módulos livres sobre $C^{\infty}\left(\mathbb{C}^{2}\right)^{\mathbb{T}^{2}}$.

Proof. Primeiramente notemos que $\partial_{x, y}$ e $\partial_{u, v}$ são $\mathbb{T}^{2}$-invariantes, $\log 0 \partial_{x, y, u, v}$ também será. Portanto:

$$
\mathfrak{X}^{3}\left(\mathbb{C}^{2}\right)^{\mathbb{T}^{2}}=\left\{f \partial_{x, y, u, v}: f \in C^{\infty}\left(\mathbb{C}^{2}\right)^{\mathbb{T}^{2}}\right\}
$$

o que certamente é um $C^{\infty}\left(\mathbb{C}^{2}\right)^{\mathbb{T}^{2}}$-modulo livre, com base $\left\{\partial_{x, y, u, v}\right\}$.

Sejam $E_{j}$ campo de Euler sobre o $j$-ésimo fator complexo, $j=1,2$, e $V_{j}$ seu respectivo campo de vetores rotacionado $\left(J\left(E_{j}\right)=V_{j}\right)$. Notemos que:

$$
\begin{gathered}
\Pi=E_{1} \wedge V_{1}+E_{2} \wedge V_{2} \\
\partial_{x, y, u, v}=\frac{1}{\left(x^{2}+y^{2}\right)\left(u^{2}+v^{2}\right)} \partial_{E_{1}, V_{1}, E_{2}, V_{2}}
\end{gathered}
$$

Os campos de vetores de Euler e rotacional são $\mathbb{S}^{1}$-invariantes, logo também os são quaisquer de seus produtos wedges. Afirmamos que podemos usar esses produtos wedges para produzir um referêncial global de trivetores invariantes, a saber:

$W_{1}:=\frac{1}{u^{2}+v^{2}} \partial_{V_{1}, E_{2}, V_{2}}, W_{2}:=\frac{1}{u^{2}+v^{2}} \partial_{E_{1}, E_{2}, V_{2}}, W_{3}:=\frac{1}{x^{2}+y^{2}} \partial_{E_{1}, V_{1}, V_{2}}, W_{4}:=\frac{1}{x^{2}+y^{2}} \partial_{E_{1}, V_{1}, E_{2}}$

em outras palavras vamos provar que dado $P \in \mathfrak{X}^{3}\left(\mathbb{C}^{2}\right)^{\mathbb{T}^{2}}$ existem $f_{j} \in C^{\infty}\left(\mathbb{C}^{2}\right)^{\mathbb{T}^{2}}, j=1,2,3,4$, tais que:

$$
P=\sum_{j=1}^{4} f_{j} W_{j}
$$

Com efeito, seja $P \in \mathfrak{X}^{3}\left(\mathbb{C}^{2}\right)^{\mathbb{T}^{2}}$. Por $P$ ser campo de trivetores em $\mathbb{C}^{2}$ segue que:

$$
P=g_{1} \partial_{y, u, v}+g_{2} \partial_{x, u, v}+g_{3} \partial_{x, y, v}+g_{4} \partial_{x, y, u}, \quad g_{i} \in C^{\infty}\left(\mathbb{C}^{2}\right)
$$

Podemos reescrever $P$ como

$$
P=\left(g_{2} \partial_{x}+g_{1} \partial_{y}\right) \wedge \partial_{u, v}+\left(g_{4} \partial_{u}+g_{3} \partial_{v}\right) \wedge \partial_{x, y}
$$


Por $P$ ser $\mathbb{T}^{2}$-invariante and

- $\partial_{x, y}$ e $\partial_{u, v}$ serem $\mathbb{T}^{2}$-invariantes;

- $\left(\rho_{g}\right)_{*}\left(g_{2} \partial_{x}+g_{1} \partial_{y}\right) \in \operatorname{span}_{\mathbb{R}}\left\{\partial_{x}, \partial_{y}\right\}$, para todo $g \in \mathbb{T}^{2}$

- $\left(\rho_{g}\right)_{*}\left(g_{4} \partial_{u}+g_{3} \partial_{v}\right) \in \operatorname{span}_{\mathbb{R}}\left\{\partial_{u}, \partial_{v}\right\}$, para todo $g \in \mathbb{T}^{2} ;$

segue-se que:

$$
g_{2} \partial_{x}+g_{1} \partial_{y}, \quad g_{4} \partial_{u}+g_{3} \partial_{v} \in \mathfrak{X}^{1}\left(\mathbb{C}^{2}\right)^{T}
$$

Afirmamos que

$$
g_{2} \partial_{x}+g_{1} \partial_{y}=h_{1} E_{1}+h_{2} V_{1}, \quad h_{i} \in C^{\infty}\left(\mathbb{C}^{2}\right)^{T}
$$

A primeira observação a ser feita é que $g_{2} \partial_{x}+g_{1} \partial_{y}$ é $\mathbb{S}^{1}$-invariante com relação a ação ao primeiro fator complexo, isso implica que esse campo de vetores deve se anular ao longo do eixo $w$. Aplicando lema da divisão sobre cada $g_{i}$ resulta:

$$
g_{1}=x l_{1}+y l_{2}, \quad g_{2}=x n_{1}+y n_{2}, \quad l_{i}, n_{i} \in C^{\infty}\left(\mathbb{C}^{2}\right)
$$

logo:

$$
g_{2} \partial_{x}+g_{1} \partial_{y}=\left(x l_{1}+y l_{2}\right) \partial_{x}+\left(x n_{1}+y n_{2}\right) \partial_{y} .
$$

No complementar do eixo $w$ os campos $E_{1}$ e $V_{1}$ formam um referêncial tangênte as translações do eixo $w$. Portanto em $\mathbb{C}^{2} \backslash\{z=0\}$ :

$$
g_{2} \partial_{x}+g_{1} \partial_{y}=q_{1} E_{1}+q_{2} V_{1}
$$

Por causa que $E_{1}$ e $V_{1}$ são invariantes e linearmente independentes, $q_{1}, q_{2} \in$ $C^{\infty}\left(\mathbb{C}^{2} \backslash\{z=0\}\right)^{\mathbb{S}^{1}}$. Vamos trabalhar sobre o hiperplano $\{y=0\}$. A partir de (3.7) obtemos em $\left(\mathbb{C}^{2} \backslash\{z=0\}\right) \cap\{y=0\}$ a igualdade entre as funções

$$
x q_{1}=x l_{1}, \quad-x q_{2}=x n_{1} \Longrightarrow q_{1}=l_{1}, \quad q_{2}=-n_{1} .
$$

Por causa que $l_{1}$ e $n_{1}$ são definidos em todo $\{y=0\}$, logo $q_{1}$ e $q_{2}$ também o são. Por último, como $q_{i}$ são $\mathbb{S}^{1}$-invariantes

$$
q_{i}(x, 0, u, v)=q_{i}(-x, 0, u, v)
$$

e por resultados clássicos [Smooth functions invariant under the action of a compact Lie group] resulta que

$$
(x, y, u, v) \mapsto q_{i}\left(\sqrt{x^{2}+y^{2}}, 0, u, v\right)
$$


pertence a $C^{\infty}(\mathbb{C})^{\mathbb{T}^{2}}$. Em outras palavras mostramos que $q_{i} \in C^{\infty}(\mathbb{C} \backslash\{w=$ $0\})^{\mathbb{T}^{2}}$ se estende a $g_{i} \in C^{\infty}\left(\mathbb{C}^{2}\right)^{\mathbb{T}^{2}}$.

Lema (3.10) garante que

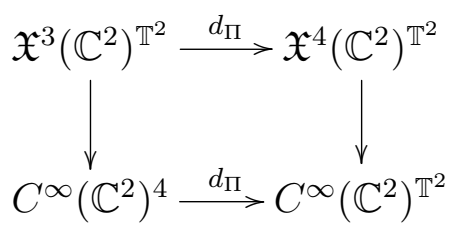

Uma simplificação adicional vem de um resultado clássico que identifica funções invariantes definidas em $\mathbb{C}^{2}$ com funções definidas no quadrante positivo em $\mathbb{R}^{2}[9]$. Seja

$$
\rho: \mathbb{C}^{2} \rightarrow \mathbb{R}^{2}, \quad(z, w) \mapsto(z \bar{z}, w \bar{w})
$$

e seja $N \subset \mathbb{R}^{2}$ quadrante positivo. Observe que a imagem de $\rho$ é exatamente $N$. Aqui lembremos que uma função $f=f\left(t_{1}, t_{2}\right): N \rightarrow \mathbb{R}$ é diferenciável se for a restrição de alguma função definida num aberto de $\mathbb{R}^{2}$ contendo $N$. Então:

$$
\rho^{*}: C^{\infty}(N) \longrightarrow C^{\infty}\left(\mathbb{C}^{2}\right)
$$

é uma bijeção sobre a imagem $C^{\infty}(N)^{\mathbb{C}^{2}}$.

Lemma 3.6 Tranferindo o complexo de Poisson para funções invariantes em $N$,

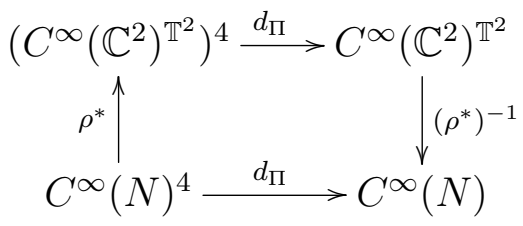

o operador $d_{\Pi}$ toma forma de

$$
(f, g, h, l) \mapsto-t_{1}^{2} \partial_{t_{1}} g-t_{2}^{2} \partial_{t_{2}} l
$$

Proof. A primeira aplicação, o pull back $\rho^{*}$, toma forma de

$(f, g, h, l) \mapsto\left(f\left(x^{2}+y^{2}, u^{2}+v^{2}\right), g\left(x^{2}+y^{2}, u^{2}+v^{2}\right), h\left(x^{2}+y^{2}, u^{2}+v^{2}\right), l\left(x^{2}+y^{2}, u^{2}+v^{2}\right)\right)$

Sabemos que $\mathfrak{X}^{3}\left(\mathbb{C}^{2}\right)^{\mathbb{T}^{2}} \simeq\left(C^{\infty}\left(\mathbb{C}^{2}\right)^{\mathbb{T}^{2}}\right)^{4}$. Como a estrutura de Poisson padrão em $\mathbb{C}$ é o produto wegde dos campos de vetor de Euler e rotacional, o produto wegde dessas duas famílias de campos de vetor são automaticamente cociclos de Poisson. Portanto, para calcular a diferença de Poisson da tupla acima, devemos calcular os Hamiltonianos de 


$$
\left(\frac{f\left(x^{2}+y^{2}, u^{2}+v^{2}\right)}{u^{2}+v^{2}}, \frac{g\left(x^{2}+y^{2}, u^{2}+v^{2}\right)}{u^{2}+v^{2}}, \frac{h\left(x^{2}+y^{2}, u^{2}+v^{2}\right)}{x^{2}+y^{2}}, \frac{h\left(x^{2}+y^{2}, u^{2}+v^{2}\right)}{u^{2}+v^{2}}\right)
$$

e então o produto wegde com cada hamiltoniano com o campo multivetor de grau 3 correspondente.

$$
\begin{aligned}
d\left(\frac{f\left(x^{2}+y^{2}, u^{2}+v^{2}\right)}{u^{2}+v^{2}}\right) & =\frac{2 x \partial_{t_{1}} f}{u^{2}+v^{2}} d x+\frac{2 y \partial_{t_{1}} f}{u^{2}+v^{2}} d y++\frac{2 u \partial_{t_{1}} f\left(u^{2}+v^{2}\right)-f 2 u}{\left(u^{2}+v^{2}\right)^{2}} d u+ \\
& +\frac{2 v \partial_{t_{1}} f\left(u^{2}+v^{2}\right)-f 2 v}{\left(u^{2}+v^{2}\right)^{2}} d v
\end{aligned}
$$

E sua hamiltoniana é

$$
\frac{2 x\left(x^{2}+y^{2}\right) \partial_{t_{1}} f}{u^{2}+v^{2}} \partial_{y}-\frac{2 y\left(x^{2}+y^{2}\right) \partial_{t_{1}} f}{u^{2}+v^{2}} \partial_{x}+\frac{2 u \partial_{t_{1}} f\left(u^{2}+v^{2}\right)-f 2 u}{u^{2}+v^{2}} \partial_{v}-\frac{2 v \partial_{t_{1}} f\left(u^{2}+v^{2}\right)-f 2 v}{u^{2}+v^{2}} \partial_{u},
$$

Aqui temos que cunhar com $R_{1} \wedge E_{2} \wedge R_{2}$. As primeiras duas somas já são múltiplos de $R_{1}$ e as duas últimas somas são tangentes a $\partial_{u, v}$. Portanto, o que temos é um cociclo de Poisson. A situação para $g$ é diferente porque temos o produto wedge $E_{1} \wedge E_{2} \wedge R_{2}$, e o resultado é:

$$
-2\left(x^{2}+y^{2}\right) \partial_{t_{1}} g \partial_{x, y, u, v}
$$

Portanto o operador $d_{\Pi}$ torna-se:

$$
(f, g, h, l) \mapsto\left(-2 t_{1}^{2} \partial_{t_{1}} g-2 t_{2}^{2} \partial_{t_{2}} l\right)
$$

\section{Proof. Teorema 3.6}

Pelos resultados provados anteriormente apenas precisamos calcular o quociente de $C^{\infty}(N)$ pelo ideal gerado pelas funções $t_{1}^{2}$ e $t_{2}^{2}$. Afirmamos que esse quociente é isomorfo a $\mathbb{R}^{4}$ e possui como base as funções

$$
1, t_{1}, t_{2}, t_{1} t_{2}
$$

Com efeito, seja $p \in C^{\infty}(N)$. Definimos:

$p_{0}=p(0,0), \quad p_{1}=p\left(t_{1}, 0\right)-p_{0}, \quad p_{2}=p\left(0, t_{2}\right)-p_{0}, \quad p_{3}=p-\left(p_{0}+p_{1}+p_{2}\right)$

e mais uma vez temos $p=p_{0}+p_{1}+p_{2}+p_{3}$. Novamente aplicando lema da divisão e lema (3.8) podemos escrever

$$
p=p_{0}+t_{1} l_{1}+t_{2} l_{2}+t_{1} t_{2} l_{3}, \quad l_{i} \in C^{\infty}(N)
$$


Vamos repetir a mesma decomposição, mas agora para $l_{1}, l_{2}$ e $l_{3}$ e reagrupar os termos para obter:

$$
p=p_{0}+a_{1} t_{1}+a_{2} t_{2}+t_{1}^{2} m_{1}+t_{1} t_{2} m_{2}+t_{2}^{2} m_{3}, \quad m_{i} \in C^{\infty}(N)
$$

onde $p_{0}, a_{1}$ e $a_{2}$ são constantes. Fazendo o mesmo para função $m_{2}$ resulta em

$$
p=p_{0}+a_{1} t_{1}+a_{2} t_{2}+a_{3} t_{1} t_{2}+t_{1}^{2} n_{1}+t_{2}^{2} n_{2}, \quad n_{i} \in C^{\infty}(N)
$$

onde $a_{3}$ é também constante, o que finaliza a prova do teorema.

Terminamos esse capítulo com algumas especulações sobre o cálculo completo da cohomologia de Poisson de $\left(\mathbb{C}^{n}, \Pi\right)$, usando nossos métodos:

1. Pode-se recuperar os resultados de Nakanishi sobre $H_{\Pi}^{\bullet}(\mathbb{C})$ com nossa abordagem. Os cociclos de grau zero são funções constantes; Para o cálculo de $H^{1}$ verifica-se imediatamente se os campos vetoriais fechados pertencem ao submódulo onde se pode aplicar o operador de homotopia relacionado ao operador de média. O cálculo da cohomologia invariante $H_{\Pi}^{\bullet}(\mathbb{C})^{\mathbb{S}^{1}}$ usando funções definidas na semireta positiva é imediato.

2. Em geral o teorema de Kunneth para cohomologia de De Rham não é válido para cohomologia de Poisson. Entretando, nossa expectativa é que

$$
H_{\pi}^{\bullet}\left(\mathbb{C}^{n}\right) \cong H_{\pi}^{\bullet}(\mathbb{C}) \otimes n
$$

Para provar a afirmação precisaríamos:

- Generalizar o lema da divisão para funções sobre $\mathbb{C}^{n}$ o que é uma processo padrão;

- Generalizar o Teorema (3.9) para todos os grupos de cohomologia de Poisson. Essa é a parte mais complicada. Para caso geral a cohomologia top parece ser canônica a generalização, porém para os outros casos outras complicações podem aparecer;

- Generalizar lema (3.10) para multivetores em $\mathbb{C}^{n}$

- Generalizar os cálculos do complexo induzido em módulos livres de funções no quadrante positivo. 


\section{4 \\ Deformação}

Neste capítulo abordaremos o problema-chave desta tese, deformações de estruturas de Poisson sobre variedades tóricas. Como já mencionado nesse manuscrito a partir de um espaço afim podemos extrair duas estruturas Poisson, de um lado, como feito no capítulo 2, uma construção GIT e utilizando bivetor quadrático obtemos estrutura Poisson-tórica sobre quociente, por outro lado temos uma construção via teorema de Delzant e redução hamiltoniana na qual geral estrutura simplética sobre o quociente. Nossa variedade em questão é $\mathbb{C P}^{n}$. A estratégia baseia-se em trabalhar com levantamentos a $\mathbb{C}^{n+1}$. Construímos uma combinação convexa $\Pi_{t}$ entre os bivetores quadrático canônico e o bivetores constante canônico, indexado por um $t \in[0,1]$ (com essa orientação). Nesse ponto verifica-se que para cada $t>0$ a estrutura em questão é simplética, além disso, a ação do toro $\mathbb{S}^{1}$ em $\left(\mathbb{C}^{n+1} \backslash\{0\}, \omega_{t}=\left(\Pi_{t}\right)^{-1}\right)$ é hamiltoniana. Utilizando-se da redução simplética e suas propriedades construímos família de bivetores a dois parâmetros em $\mathbb{C}^{n+1}$ nas quais descem a estruturas kahlerianas em $\mathbb{C P}^{n}$. Através de argumentos geométricos extraímos subfamília $\hat{\pi}_{t}$ a 1-parâmetro na qual converge a estrutura quadrática. Por último mostramos que tal deformação é não trivial.

\section{1}

\section{Problema Geral}

Seja $X(\Sigma)$ variedade tórica suave e completa, ou equivalentemente, construída a partir de uma fan completo e suave $\Sigma$, sob ação do toro $\mathbb{T}_{\mathbb{C}} \simeq$ $\left(\mathbb{C}^{*}\right)^{n}$. Seja $\Pi \in \mathfrak{X}^{2}(X(\Sigma))^{\mathbb{T}_{\mathbb{C}}}$ uma estrutura de Poisson cujas folhas de $\Pi$ são

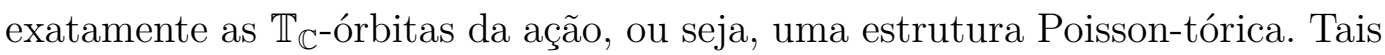
estruturas existem via construção de Arlo Caine [7]. Seja $\mathcal{O}$ órbita aberta e densa em $X$, fazendo uma identificação $\mathcal{O} \simeq \mathbb{T}_{\mathbb{C}} \simeq\left(\mathbb{C}^{*}\right)^{n}$, podemos escrever

$$
\left.\Pi\right|_{\mathcal{O}} \in \mathfrak{X}^{2}(\mathcal{O})^{\mathbb{T}_{\mathbb{C}}} \Rightarrow \Pi^{\prime} \in \mathfrak{X}^{2}\left(\left(\mathbb{C}^{*}\right)^{n}\right)^{\left(\left(\mathbb{C}^{*}\right)^{n}, *\right)}
$$

a estrutura $\Pi^{\prime}$, que por conveniência vamos escrever simplesmente $\Pi$, independende da identificação $\mathcal{O} \simeq\left(\mathbb{C}^{*}\right)^{n}$.

Tendo em vista que exp : $\mathbb{C}^{n} \longrightarrow\left(\mathbb{C}^{*}\right)^{n}$ é difeomorfismo local, temos mapa linear $\exp ^{*}: \mathfrak{X}^{*}\left(\left(\mathbb{C}^{*}\right)^{n}\right) \longrightarrow \mathfrak{X}^{*}\left(\mathbb{C}^{n}\right)$. Desta forma, podemos fazer o 
pullback do bivetor $\Pi$ em $\left(\mathbb{C}^{*}\right)^{n}$ e definir:

$$
\pi:=\exp ^{*}(\Pi) \in \mathfrak{X}^{2}\left(\mathbb{C}^{n}\right)
$$

A invariancia de $\Pi$ em $\left(\left(\mathbb{C}^{*}\right)^{n}, *\right)$ garante a invariancia de $\pi$ em $\left(\mathbb{C}^{n},+\right)$, ou seja, $\pi$ é bivetor constante em $\mathbb{C}^{n}$.

Suporemos $\pi$ ser limite de estruturas kahlerianas $\pi_{t}$ :

$$
\pi_{0}:=\pi \leftarrow \pi_{t} \in \mathfrak{X}^{2}\left(\mathbb{C}^{n}\right) \text {, para } t \longrightarrow 0^{+}
$$

Podemos a partir de agora nos fazer algumas perguntas.

- Quais propriedades $\pi$ possui?

- Quais estruturas $\Pi \in \mathfrak{X}^{2}(X(\Sigma))^{\mathbb{T}_{\mathbb{C}}}$ admintem "pullback" $\pi$ tendo a propriedade (4.1), ou seja, sendo limite de bivetores Kahlerianos?

- Em quais contextos a deformação $\pi_{t} \rightarrow \pi$ induz deformação $\Pi_{t} \rightarrow \Pi \mathrm{em}$ $X(\Sigma) ?$

- Quais condições necessárias para a exponencial de uma estrutura tórica possa se estender a todo $X(\Sigma)$ ?

Em geral tais perguntas são difíceis de serem respondidas com tamanho grau de generalidade. Esse é um dos motivos de o porquê restringimos nossa atenção a variedade tórica $\mathbb{C P}^{n}$ e estudarmos deformação da estrutura Poisson-tórica. Além disso no espaço projetivo as duas construções ficam "mais próximas" o que facilita a obtenção da deformação. Vamos agora rever brevemente cada um desses processos.

\section{2}

\section{Construção via Delzant}

Seja $\Delta \subset\left(\mathbb{R}^{n}\right)^{*}$ polítopo de Delzant, definição (2.6.1), com $d$ facetas. Sejam $v_{i} \in \mathbb{Z}^{n}, i=1, \ldots, d$, os vetores normais primitivos apontando para fora para as facetas. Existem $\lambda_{i} \in \mathbb{R}$ de modo que:

$$
\Delta=\left\{x \in\left(\mathbb{R}^{n}\right)^{*} \mid\left\langle x, v_{i}\right\rangle \leq \lambda_{i}, i=1, \ldots, d\right\}
$$

Seja $e_{1}, \ldots, e_{d} \in \mathbb{R}^{d}$ base canônica e consideremos a aplicação:

$$
\begin{gathered}
\pi: \mathbb{R}^{d} \longrightarrow \mathbb{R}^{n} \\
e_{i} \longmapsto v_{i}
\end{gathered}
$$


Notemos que $\pi$ é sobrejetiva e mapea $\mathbb{Z}^{d}$ em $\mathbb{Z}^{n}$, [11]. Desta forma $\pi$ induz mapa sobrejetivo, que continuaremos denotando por $\pi$, entre os toros:

$$
\mathbb{T}^{d}=\mathbb{R}^{d} / \mathbb{Z}^{d} \stackrel{\pi}{\longrightarrow} \mathbb{R}^{n} / \mathbb{Z}^{n}=\mathbb{T}^{n} \longrightarrow 0
$$

Sejam

$$
\begin{aligned}
N & :=\text { kernel de } \pi\left(N \text { é subgroupo de Lie de } \mathbb{T}^{d}\right) \\
\mathfrak{n} & :=\text { álgebra de Lie de } N \\
\mathbb{R}^{d} & :=\text { álgebra de Lie de } \mathbb{T}^{d} \\
\mathbb{R}^{n} & :=\text { álgebra de Lie de } \mathbb{T}^{n} .
\end{aligned}
$$

A sequência exata de toros

$$
0 \longrightarrow N \stackrel{i}{\longrightarrow} \mathbb{T}^{d} \stackrel{\pi}{\longrightarrow} \mathbb{T}^{n} \longrightarrow 0
$$

onde $i$ é inclusão, induz sequência exata de álgebras de Lie

$$
0 \longrightarrow \mathfrak{n} \stackrel{i}{\longrightarrow} \mathbb{R}^{d} \stackrel{\pi}{\longrightarrow} \mathbb{R}^{n} \longrightarrow 0
$$

com sequencia dual exatada dual

$$
0 \longrightarrow\left(\mathbb{R}^{n}\right)^{*} \stackrel{\pi^{*}}{\longrightarrow}\left(\mathbb{R}^{d}\right)^{*} \stackrel{i^{*}}{\longrightarrow} \mathfrak{n}^{*} \longrightarrow 0
$$

Agora considere $\mathbb{C}^{d}$ com a forma simplética canônica $\omega_{0}=\frac{i}{2} \sum_{k=1}^{d} d z_{k} \wedge$ $d \bar{z}_{k}$ juntamente com a ação hamiltoniana de $\mathbb{T}^{d}$

$$
\left(e^{2 \pi i t_{1}}, \ldots, e^{2 \pi i t_{d}}\right) \cdot\left(z_{1}, \ldots, z_{d}\right)=\left(e^{2 \pi i t_{1}} z_{1}, \ldots, e^{2 \pi i t_{d}} z_{d}\right)
$$

seu mapa momento $\phi: \mathbb{C}^{d} \longrightarrow\left(\mathbb{R}^{d}\right)^{*}$ é dado por

$$
\phi\left(z_{1}, \ldots, z_{d}\right)=-\frac{1}{2}\left(\left|z_{1}\right|^{2}, \ldots,\left|z_{d}\right|^{2}\right)+\left(\lambda_{1}, \ldots, \lambda_{d}\right)
$$

Esses elementos juntos $\left(\mathbb{C}^{d}, \omega, \mathbb{T}^{d}, \phi\right)$ formam o que chamamos de um $\mathbb{T}^{d}$-espaço hamiltoniano. Como $N \leqslant \mathbb{T}^{d}$ é subgrupo de Lie, podemos restringir ação de $\mathbb{T}^{d}$ a $N$ e obtermos assim um $N$-espaço hamiltoniano $\left(\mathbb{C}^{d}, \omega, N, \tilde{\phi}\right)$, onde $\tilde{\phi}=i^{*} \circ \phi: M \longrightarrow \mathfrak{n}^{*}$. Seja $Z=\left(i^{*} \circ \phi\right)^{-1}(0)$ conjunto de nível. Pode-se mostrar que $Z$ é compacto e $N$ age em $Z$ livremente [11]. Além disso, 0 é valor regular para $i^{*} \circ \phi, \operatorname{logo} Z$ é subvariedade de $\mathbb{C}^{d}$ de codimensão real $d-n=\operatorname{dim}\left(\mathfrak{n}^{*}\right)$. Portanto, passando ao quociente obtemos $M_{\Delta}=Z / N$ variedade compacta de 
dimensão:

$$
\operatorname{dim}_{\mathrm{R}} M_{\Delta}=d+n-\underbrace{(d-n)}_{\operatorname{dim} N}=2 n
$$

e, consequentemente, um $N$-fibrado principal sobre $M_{\Delta}$. Considere o seguinte diagrama:

$$
\begin{aligned}
& Z \stackrel{j}{\longrightarrow} \mathbb{C}^{d} \\
& p \downarrow \\
& M_{\Delta}
\end{aligned}
$$

onde $j: Z \hookrightarrow \mathbb{C}^{d}$ é a inclusão. Teorema de Marsden-Weinstein-Meyer garante a existência de uma forma $\omega_{\Delta}$ em $M_{\Delta}$ satisfazendo:

$$
p^{*} \omega_{\Delta}=j^{*} \omega_{0}
$$

\section{3}

\section{Construção via Arlo Caine}

Nesta seção vamos começar fazendo uma breve construção, baseada no

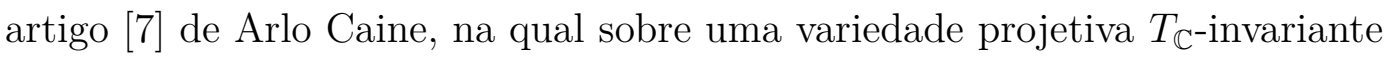

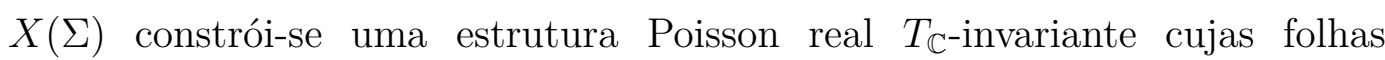

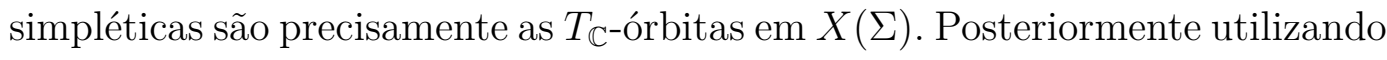
a estrutura quadrática introduzida na seção precedente vamos construir uma deformação simplética sobre $\mathbb{C P}^{n}$ utilizando redução simplética.

\section{Proposition 4.1 Sejam}

$$
\pi=-2 i \sum_{j=1}^{n} \frac{\partial}{\partial z_{j}} \wedge \frac{\partial}{\partial \bar{z}_{j}} e \Pi=-2 i \sum_{j=1}^{n} z_{j} \bar{z}_{j} \frac{\partial}{\partial z_{j}} \wedge \frac{\partial}{\partial \bar{z}_{j}}
$$

bivetores sobre $\mathbb{C}^{n}$ e $\rho_{+}$e $\rho$ as ações de adição e multiplicação de $\left(\mathbb{C}^{*}\right)^{n}$ em $\mathbb{C}^{n}$, respectivamente. Então valem:

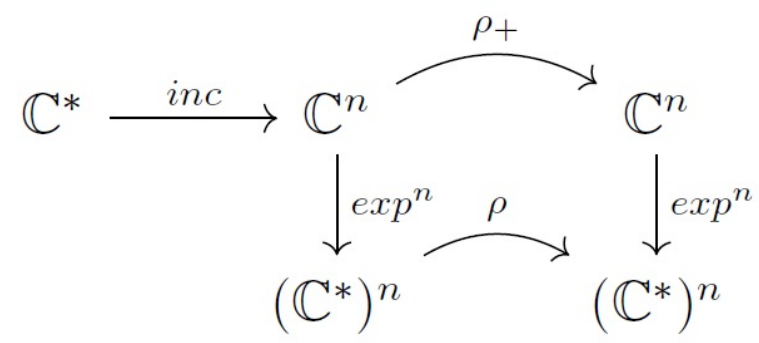

Figura 4.1: Diagrama das ações

1. $\pi$ e П são Poisson; 
2. Se $\exp ^{n}:\left(z_{1}, \ldots, z_{n}\right) \mapsto\left(\exp \left(z_{1}\right), \ldots, \exp \left(z_{n}\right)\right)$, então $\exp ^{n}{ }_{*} \pi=\Pi$;

3. $\pi$ é $\rho_{+}$-invariante e $\Pi$ é $\rho$-invariante;

Proof. $(i)$ - Para $n=1$, ou seja em $\mathbb{C}$, notamos que ambos são bivetores num espaço de dimensão 2, logo Poisson. No caso geral basta perceber que $\pi$ e $\Pi$ são bivetores Poisson induzidos pela estrutura produto em $\mathbb{C} \times \cdots \times \mathbb{C}$. (ii) segue do fato que se $\exp (z)=w$, então $\exp _{*} \frac{\partial}{\partial z}=w \frac{\partial}{\partial w} \exp _{*} \frac{\partial}{\partial \bar{z}}=\bar{w} \frac{\partial}{\partial \bar{w}}$. (iii)Claramente por $\pi$ é $\rho_{+}$invariante, como consequencia disso e pelo fato que $\exp _{*}^{n} \pi=\Pi$ resulta que o diagrama acima é comutativo.

É um fato interessante que esta estrutura quadrática $\Pi$ surja naturalmente como imagem pela exponencial de uma estrutura de Poisson constante. Em dimensão $n=1$, temos que a folheação induzida $\Pi$ é composta por duas folhas (simpléticas), a origem e seu complemento. O resultado a seguir geraliza esse fato.

Proposição 4.1 As folhas simpléticas de $\left(\mathbb{C}^{n}, \Pi\right)$ são exatamentes as órbitas de $\mathbb{T}_{\mathbb{C}}^{n}=\left(\mathbb{C}^{*}\right)^{n}$

Proposição $4.2 A$ ação de $T_{\mathbb{C}}{ }^{d}$ sobre $\left(\mathbb{C}^{d}, \Pi\right)$ é Poisson, mas não hamiltoniana. Entretanto, cada folha simplética $\left(\mathbb{C}_{0}\right)^{S}$ admite uma ação hamiltoniana de um subtoro real $\mathbb{T}^{S} \subset \mathbb{T}^{d}$.

\section{4 \\ Deformação}

Nossa variedade tórica compacta e diferenciável de interesse aqui será $\mathbb{C P}^{n-1}$. O valor $n-1$ se deve ao fato que preferimos trabalhar com seu espaço total $\mathbb{C}^{n}$ em vez de $\mathbb{C}^{n+1}$. Através das duas construções apresentadas nas seções precedentes, existem duas estruturas Poisson sobre $\mathbb{C P}^{n-1}$. Primeira estrutura proveniente da forma simplética canônica em $\mathbb{C}^{n}$ via redução simplética, logo um "reescalamento" da estrutura Fubini-Study e a outra proveniente da construção de Arlo Cane através do quociente da estrutura quadrática canônica. Nesse momento podemos nos perguntar como podemos comparar tais estruturas, ou mais, como construir, se possível, família de estruturas Poisson lincado-as?

Respondendo a essas perguntas, ao final desta seção teremos provado o seguinte teorema: 
Teorema 4.3 Existe uma deformação não-trivial da estrutura quadrática canônica de $\mathbb{C P}^{n}$ na qual é limite contínuo de estruturas kahlerianas. Além disso, mostra-se que a ação do toro $\mathbb{T}^{n}$ em $\mathbb{C P}^{n}$ é hamiltoniana para cada uma dessas estruturas kahlerianas.

Vamos a construção. Sabemos que a ação diagonal $\rho: \mathbb{S}^{1} \curvearrowright\left(\mathbb{C}^{n}, \omega_{\text {std }}\right)$, $\rho\left(\zeta,\left(z_{1}, \ldots, z_{n}\right)\right)=\left(\zeta \cdot z_{1}, \ldots, \zeta \cdot z_{n}\right)$, é ação hamiltoniana com mapa momento $\mu: \mathbb{C}^{n} \longrightarrow \mathbb{R}^{*} \simeq \mathbb{R}$, dado por

$$
\mu\left(z_{1}, \ldots, z_{n}\right)=-\frac{1}{2} \sum_{j=1}^{n}\left|z_{j}\right|^{2}
$$

Para cara escolha de $\xi<0$ temos que $\mu^{-1}(\xi)$ é uma esfera em $\mathbb{C}^{n}$. $\rho$ age livremente em $\mu^{-1}(\xi)$, por redução simplética para cada escolha de tal $\xi$ existe forma simplética $\omega_{\xi} \in \Omega\left(\mu^{-1}(\xi) / S^{1}\right)$ de modo que $i^{*} \omega_{s t d}=p^{*} \omega_{\xi}$, onde $i: \mu^{-1}(\xi) \hookrightarrow \mathbb{C}^{n}$ é a inclusão e $p: \mu^{-1}(\xi) \rightarrow \mu^{-1}(\xi) / \mathbb{S}^{1}$ é quociente. Observemos o seguinte diagrama: onde $\rho$ do lado direito estende ação diagonal de $\mathbb{S}^{1}$ para

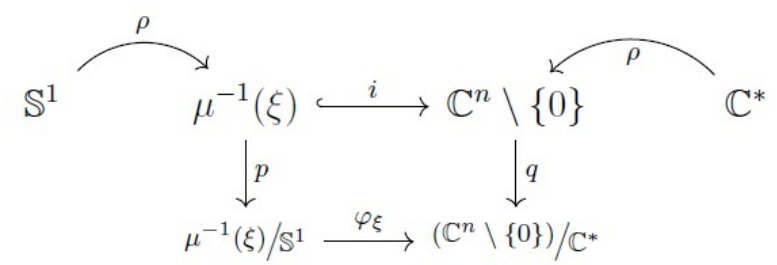

Figura 4.2: Diagrama comutativo

$\mathbb{C}^{*}$. Como podemos ver existe um difeomorfismo canônico $\varphi_{\xi}$ na qual torna o diagrama comutativo. Podemos dessa forma definir:

$$
\begin{aligned}
& \pi_{\xi}:=\left(\omega_{\xi}\right)^{-1} \in \mathfrak{X}^{2}\left(\mu^{-1}(\xi) / \mathrm{S}^{1}\right) \text { Poisson } \\
& \hat{\pi}_{\xi}:=\left(\varphi_{\xi}\right)_{*} \pi_{\xi} \in \mathfrak{X}^{2}\left(\left(\mathbb{C}^{n} \backslash 0\right) / \mathbb{C}^{*}\right) \text { Poisson }
\end{aligned}
$$

família de estruturas Poisson (não-degeneradas) $\left\{\hat{\pi}_{\xi}\right\}_{\xi<0}$ sobre $\mathbb{C P}^{n-1}$ indexadas por $\xi$. Mas novamente, como podemos comparar tais bivetores com a estrutura quadrática induzada em $\mathbb{C P}^{n-1}$ ? Uma maneira de fazermos isso é através do espaço afim $\mathbb{C}^{n}$, ou melhor, fazendo levantamentos via $q$ e comparando-os. Vamos primeiramente construir tais levantamentos.

Para cada $\xi<0$ precisamos encontrar $\Pi_{\xi} \in \mathfrak{X}^{2}\left(\mathbb{C}^{n} \backslash\{0\}\right)$ de modo que:

- $\Pi_{\xi}$ seja $\mathbb{C}^{*}$-invariante;

- $q_{*}\left(\Pi_{\xi}\right)=\hat{\pi}_{\xi}$

Pela relação $i^{*} \omega_{s t d}=p^{*} \omega_{\xi}$ sabemos que $\omega_{\xi}$ provem da restrição de $\omega_{s t d}$ para $\mu^{-1}(\xi)$ e depois "quocientada" via ação $\mathbb{S}^{1}$. Analogamente, pela mesma 
relação, para obtermos os bivetores $\Pi_{\xi}$, podemos inverter a forma canônica $\omega_{\text {std }} \in \Omega^{2}\left(\mathbb{C}^{n-1} \backslash\{0\}\right)$ obtendo bivetor não-degenerado $\Pi_{\text {std }}$ e restringi-lo a $\mu^{-1}(\xi)$. "Quocientando" $\left.\Pi_{s t d}\right|_{\mu^{-1}(\xi)}$ via ação $\mathbb{S}^{1}$ obtemos $\pi_{\xi}$. Desta forma, sabemos que $\Pi_{\xi}$ é a propagação $\mathbb{C}^{*}$-invariante de $\left.\Pi_{s t d}\right|_{\mu^{-1}(\xi)}$ a todo $\mathbb{C}^{n} \backslash\{0\}$.

Para obtermos explicitamente tais bivetores faremos uso do seguinte lema.

Lemma 4.2 Seja $P \in \mathfrak{X}^{2}\left(\mathbb{C}^{n} \backslash\{0\}\right)^{\mathbb{C}^{*}}$ e $P=\sum_{j=1}^{n} f_{j}(z) \frac{\partial}{\partial z_{j}} \wedge \frac{\partial}{\partial \bar{z}_{j}}$. Entãa $f_{j}(z)$ é homogênea de grau 2, para todo $j$.

\section{Proof.}

Pela $\mathbb{C}^{*}$-invariância temos $\left(\rho_{g}\right)_{*, z}(P(z))=P(g z)$, logo:

$$
\begin{aligned}
\left(\rho_{g}\right)_{*, z}(P(z)) & =\sum_{j=1}^{n} f_{j}(z) g \bar{g} \frac{\partial}{\partial z_{j}} \wedge \frac{\partial}{\partial \bar{z}_{j}} \\
P(g z) & =\sum_{j=1}^{n} f_{j}(g z) \frac{\partial}{\partial z_{j}} \wedge \frac{\partial}{\partial \bar{z}_{j}}
\end{aligned}
$$

o que mostra que $f_{j}(g z)=g \bar{g} f_{j}(z)$ para todo $g, z$ e $j$ onde estão definidos.

Observação 4.4 Notemos que se $f: \mathbb{C}^{n} \backslash\{0\} \rightarrow \mathbb{C}$ for homogênea de grau 2, então para todo $z \in \mathbb{C}^{n} \backslash\{0\}$ e $\theta \in \mathbb{R}^{*}$, vale a seguinte relação:

$$
f(z)=\frac{\theta^{2}}{\theta^{2}} f(z)=\frac{f(\theta z)}{\theta^{2}}
$$

Pois bem, antes de proseguirmos vamos a um fato importante. Fixado $\xi<0$, para cada $z \in \mathbb{C}^{n} \backslash\{0\}$ existe único $\theta=\theta(\xi, z)>0$ tal que $\theta z \in \mu^{-1}(\xi)$, isso definine $\theta: \mathbb{C}^{n} \backslash\{0\} \rightarrow \mathbb{R}_{>0}$.

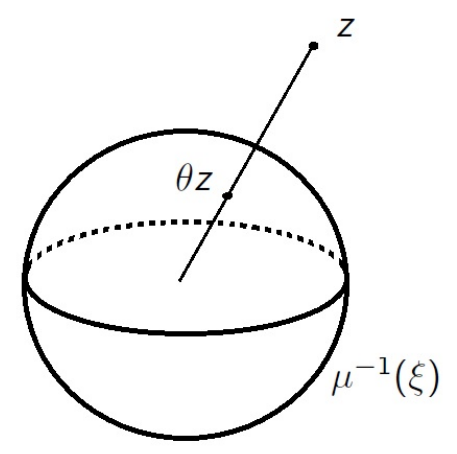

Figura 4.3: Dilatação

tal função desempenhará papel fundamental nesta construção. Como $\prod_{\xi}$ é a propagação $\mathbb{C}^{*}$-invariante de $\left.\Pi_{s t d}\right|_{\mu^{-1}(\xi)}$ para todo $\mathbb{C}^{n} \backslash\{0\}$, dado $z \in \mathbb{C}^{n} \backslash\{0\}$ 
temos $\theta z=\theta(\xi, z) z \in \mu^{-1}(\xi), \log \mathrm{O}$

$$
\Pi_{\xi}(\theta z)=\left.\left.(-2 i) \sum_{j=1}^{n} \frac{\partial}{\partial z_{j}}\right|_{\theta z} \wedge \frac{\partial}{\partial \bar{z}_{j}}\right|_{\theta z}
$$

pelo lema e pela observação chegamos a expressão geral de $\Pi_{\xi}$ :

$$
\begin{aligned}
\Pi_{\xi}(z) & =\left.\left.(-2 i) \frac{1}{\theta^{2}} \sum_{j=1}^{n} \frac{\partial}{\partial z_{j}}\right|_{z} \wedge \frac{\partial}{\partial \bar{z}_{j}}\right|_{z} \\
& =\left.\left.i \frac{|z|^{2}}{\xi} \sum_{j=1}^{n} \frac{\partial}{\partial z_{j}}\right|_{z} \wedge \frac{\partial}{\partial \bar{z}_{j}}\right|_{z}
\end{aligned}
$$

onde:

$$
\left(\frac{|\theta z|^{2}}{-2}=\xi, \quad \theta^{2}=\frac{-2 \xi}{|z|^{2}}\right)
$$

o fator $|z|^{2}$ torna explícito a $\mathbb{C}^{*}$-invariância do bivetor $\Pi_{\xi}$ e, por construção, $q_{*}\left(\Pi_{\xi}\right)=\hat{\pi_{\xi}}$.

Essa primeira construção nos é útil por caráter didático, facilitar a compreensão dos passos tomados e nos dar mais intuição para o caso geral. Pois bem, vamos a ele, para o caso geral vamos nos utilizar das duas construções (Delzant e Arlo Caine) para extrair família de estruturas Kahlerianas. Consideremos os seguintes bivetores em $\mathbb{C}^{n} \backslash\{0\}$ :

$$
\begin{aligned}
& \Pi_{0}:=\pi_{0}:=(-2 i) \sum_{j=1}^{n} z_{j} \overline{z_{j}} \frac{\partial}{\partial z_{j}} \wedge \frac{\partial}{\partial \overline{z_{j}}}, \quad \Pi_{1}:=\pi_{1}:=(-2 i) \sum_{j=1}^{n} \frac{\partial}{\partial z_{j}} \wedge \frac{\partial}{\partial \overline{z_{j}}} \\
& \pi_{t}:=(-2 i) \sum_{j=1}^{n}\left((1-t) z_{j} \overline{z_{j}}+t\right) \frac{\partial}{\partial z_{j}} \wedge \frac{\partial}{\partial \overline{z_{j}}}=(-2 i) \sum_{j=1}^{n} r_{t}\left(z_{j}\right) \frac{\partial}{\partial z_{j}} \wedge \frac{\partial}{\partial \overline{z_{j}}} .
\end{aligned}
$$

onde $t \in[0,1]$. Observa-se que $\pi_{t}$ é a combinação convexa entre $\Pi_{0}$ e $\Pi_{1}$. Uma primeira constatação a ser feita é verificar para quais valores de $t$ temos não-degenerecência, ou seja, $\pi_{t}$ é simplético. Por ser estrutura produto é fácil ver que $\pi_{t}$ é simplético para todo $t>0$ e que $\pi_{0}$ é simplético no aberto denso $\left\{z_{1} \cdots z_{n} \neq 0\right\} \subset \mathbb{C}^{n}$. A outra observação a ser feita é que apenas $\Pi_{0}$ é $\mathbb{C}^{*}$-invariante em $\mathbb{C}^{n} \backslash\{0\}$.

Afirmamos que para $t>0$ a seguinte ação é hamiltoniana:

$$
\left.\mathbb{T}^{n} \curvearrowright\left(\mathbb{C}^{n} \backslash\{0\}, \omega_{t}:=\left(\pi_{t}\right)^{-1}=\frac{i}{2} \sum_{j=1}^{n} \frac{1}{r_{t}\left(z_{j}\right)}\right) d z_{j} \wedge d \overline{z_{j}}\right)
$$

Para facilitar os cálculos vamos assumir $n=1$ e deduzir mapa momento de $\mathbb{S}^{1} \curvearrowright\left(\mathbb{C}, \omega_{t}=-\frac{i}{2} \frac{1}{r_{t}(z)} d z \wedge d \bar{z}\right)$. Seja $v=1 \in \mathbb{R} \simeq \mathbb{R}^{*}$. Claramente o campo fundamental a ação $X^{v}$ é o rotacional de Euler. Logo: 


$$
\left\{\begin{array}{l}
\iota_{X^{v}} \omega_{t}=\omega_{t}\left(X^{v}, \cdot\right)=\frac{i}{2} \frac{1}{r_{t}(z)} d z \wedge d \bar{z}\left(i z \frac{\partial}{\partial z}-i \bar{z} \frac{\partial}{\partial \bar{z}}, \cdot\right)=-\frac{1}{2} \frac{z}{r_{t}(z)} d \bar{z}-\frac{1}{2} \frac{\bar{z}}{r_{t}(z)} d z \\
d \mu^{1}=\frac{\partial \mu}{\partial z} d z+\frac{\partial \mu}{\partial \bar{z}} d \bar{z}
\end{array}\right.
$$

Igualando as derivadas parciais e integrando:

$$
\left\{\begin{array}{l}
\frac{\partial \mu}{\partial z}=-\frac{1}{2} \frac{\bar{z}}{(1-t) z \bar{z}+t} \\
\frac{\partial \mu}{\partial \bar{z}}=-\frac{1}{2} \frac{z}{(1-t) z \bar{z}+t}
\end{array} \quad \rightsquigarrow \mu_{t}(z)=-\frac{1}{2} \frac{1}{1-t} \ln \left(r_{t}(z)\right)=-\frac{1}{2} \frac{1}{1-t} \ln ((1-t) z \bar{z}+t)\right.
$$

Uma vez que $\mathbb{S}^{1}$ é grupo de Lie abeliano a equivariancia é dada por $\mu \circ \rho_{g}=\mu \mathrm{e}$, por sua vez, verificada pela invariancia da função normal $f(z)=|z|^{2}$. Voltando ao caso geral, sendo $\omega_{t}$ estrutura produto, temos $\mathbb{T}^{n} \curvearrowright\left(\mathbb{C}^{n} \backslash\{0\}, \omega_{t}\right)$ é ação hamiltoniana com mapa momento:

$$
\mu_{t}=-\frac{1}{2} \frac{1}{1-t} \ln \left(\prod_{j=1}^{n} r_{t}\left(z_{j}\right)\right)
$$

Agora, como podemos ver, estamos em situação semelhante à abordada no início desta seção. Vamos aos levantamentos! Como feito anteriormente temos o seguinte diagrama comutativo:

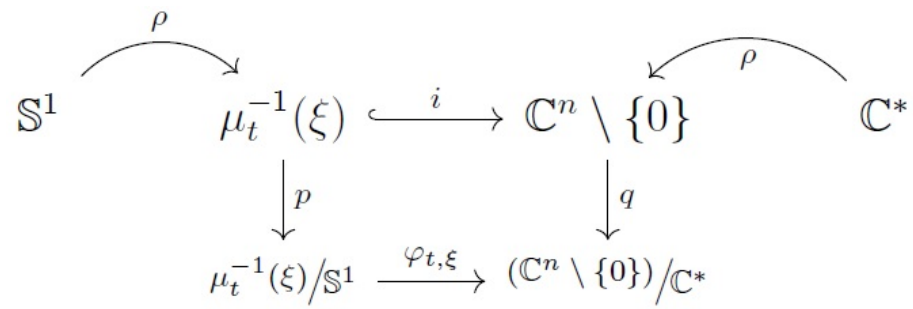

Figura 4.4: Diagrama comutativo

e família de estruturas simplética provenientes de redução simplética $\omega_{t, \xi} \in \Omega^{2}\left(\mu_{t}^{-1}(\xi) / \mathbb{S}^{1}\right)$. Definimos:

$$
\begin{gathered}
\pi_{t, \xi}:=\left(\omega_{t, \xi}\right)^{-1} \in \mathfrak{X}^{2}\left(\mu_{t}^{-1}(\xi) / \mathbb{S}^{1}\right) \text { Poisson } \\
\hat{\pi}_{t, \xi}:=\left(\varphi_{t, \xi}\right)_{*}\left(\pi_{t, \xi}\right) \in \mathfrak{X}^{2}\left(\mathbb{C}^{n} \backslash\{0\} / \mathbb{C}^{*}\right) \text { Poisson }
\end{gathered}
$$

Para cada valor de $t \in(0,1]$ e $\xi<0$, vamos encontrar $\Pi_{t, \xi} \in \mathfrak{X}^{2}\left(\mathbb{C}^{n} \backslash\{0\}\right)$ de modo que:

- $\Pi_{t, \xi}$ é $\mathbb{C}^{*}$-invariante;

- $q_{*}\left(\Pi_{t, \xi}\right)=\hat{\pi}_{t, \xi} \in \mathfrak{X}^{2}\left(\mathbb{C P}^{n-1}\right)$ 
Para encontrarmos tais levantamentos precisamos compreender a geometria de $\mu_{t}^{-1}(\xi)$. Aqui começamos a encontrar nossas primeiras dificuldades, pois enquanto $\mu_{0}^{-1}(\xi)=\mu^{-1}(\xi) \subset \mathbb{C}^{n}$ era uma esfera agora o conjunto de nível $\mu_{t}^{-1}(\xi)$ é dado por:

$$
\mu_{t}^{-1}(\xi)=\left\{z=\left(z_{1}, \ldots, z_{n}\right) \in \mathbb{C}^{n}: \prod_{j=1}^{n} r_{t}\left(z_{j}\right)=e^{-2 \xi(1-t)}\right\} \simeq \mathbb{S}^{2 n-1} \subset \mathbb{C}^{n}
$$

Para termos um pouco de intuição sobre esses conjuntos de níveis, vamos supor $n=2$ e observar $\left\{\mu_{t}^{-1}(\xi) \cap \mathbb{R}^{2}\right\}_{\xi<0}$ para $t=1, t=1 / 2$ e $t=1 / 4$, respectivamente:

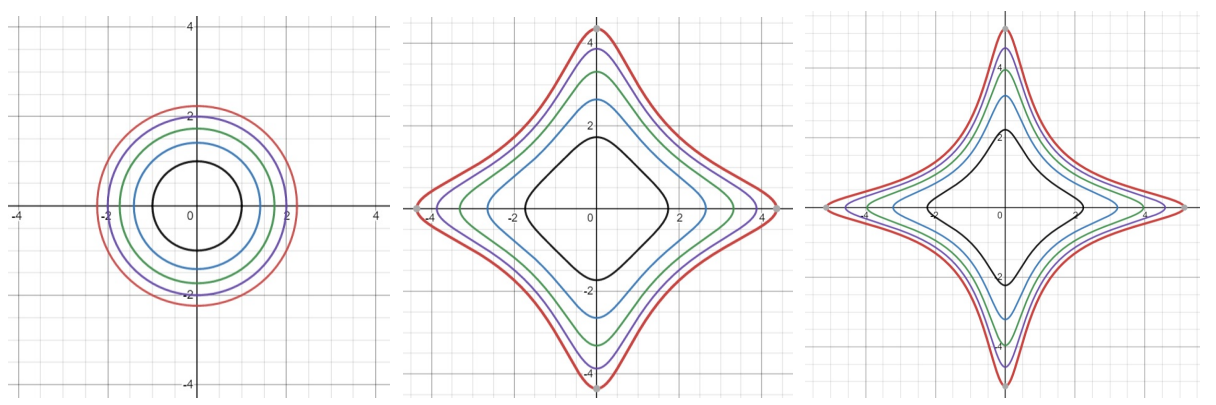

Figura 4.5: Conjuntos de níveis

Analogamente ao que foi feito antes para cada escolha de $\xi<0, t \in(0,1]$ e $z \in \mathbb{C}^{n} \backslash\{0\}$ existe único $\theta=\theta(t, \xi, z)>0$ de modo que $\theta z \in \mu_{t}^{-1}(\xi)$, isso define $\theta=\theta(\xi, t): \mathbb{C}^{n} \backslash\{0\} \longrightarrow \mathbb{R}_{>0}$. Observe que obter $\theta(t, \xi, z)$ significa encontrar raiz positiva do seguinte polinômio:

$$
\left((1-t) \theta^{2} z_{1} \bar{z}_{1}+t\right) \cdots\left((1-t) \theta^{2} z_{n} \bar{z}_{n}+t\right)=e^{-2(1-t) \xi}
$$

porém vale ressaltar que as raízes de um polinômio complexo dependem continuamente dos coeficientes, mas não suavemente. A título de curiosidade para $n=2$ temos a seguinte expressão de $\theta$ :

$$
\theta(t, \xi, z)=\sqrt{\frac{-t\left(\left|z_{1}\right|^{2}+\left|z_{2}\right|^{2}\right)+\sqrt{t^{2}\left(\left|z_{1}\right|^{2}-\left|\omega_{2}\right|^{2}\right)^{2}+4\left|z_{2}\right|^{2}\left|z_{1}\right|^{2} e^{-2(1-t) \xi}}}{2(1-t)\left|z_{1}\right|^{2}\left|z_{z}\right|^{2}}}
$$

Mais uma vez, estamos interessados nos levantamentos, ou seja, para cada $\xi<0$ e $t \in(0,1]$ queremos encontrar $\Pi_{t, \xi} \in \mathfrak{X}^{2}\left(\mathbb{C}^{n} \backslash\{0\}\right)$ de modo que:

- $\Pi_{t, \xi} \in \mathfrak{X}^{2}\left(\mathbb{C}^{n} \backslash\{0\}\right)$ seja $\mathbb{C}^{*}$-invariante;

- $q_{*}\left(\Pi_{t, \xi}\right)=: \hat{\pi}_{t, \xi}$ 
utilizando-se da relação $i^{*} \omega_{t}=p^{*} \omega_{t, \xi}$, onde $i: \mu_{t}^{-1}(\xi) \hookrightarrow \mathbb{C}^{n}$ é a inclusão e $p: \mu_{t}^{-1}(\xi) \rightarrow \mu_{t}^{-1}(\xi) / \mathbb{S}^{1}$ é a projeção canônica, sabemos que $\Pi_{t, \xi}$ é a propagação $\mathbb{C}^{*}$-invariante de $\left.\Pi_{t}\right|_{\mu_{t}^{-1}(\xi)}$ para todo $\mathbb{C}^{n} \backslash\{0\}$. Aplicando lema 4.2 e observação 4.4 neste contexto chegaremos a seguinte expressão:

$$
\Pi_{t, \xi}(z)=(-2 i) \sum_{j=1}^{n}\left((1-t) z_{j} \bar{z}_{j}+\frac{t}{\theta^{2}}\right) \partial z_{j} \wedge \partial \bar{z}_{j}
$$

Como podemos ver toda análise de convergência e diferenciabilidade sobre $\Pi_{t, \xi}$ está associada a análise de $\frac{t}{\theta^{2}}$. Uma relação imediata quando $t \longrightarrow 0^{+}$ é a seguinte:

$$
\Pi_{t, \xi} \longrightarrow \Pi_{0} \Leftrightarrow \frac{t}{\theta^{2}} \longrightarrow 0
$$

Aqui nos esbarramos num problema. Como queremos família a 1-parâmetro, se $\xi=\xi(t)$ é qualquer, então em geral $\frac{t}{\theta^{2}} \nrightarrow 0$. Nosso objetivo agora é extrair $\xi(t), t \in(0,1]$, de modo que:

$$
\frac{t}{\theta^{2}} \longrightarrow 0 \text { uniformemente em compactos de }[0,1] \times\left(\mathbb{C}^{n} \backslash\{0\}\right)
$$

e, consequêntemente, $\Pi_{t, \xi(t)} \rightarrow \Pi_{0}$. E por fim, "descendo":

$$
\hat{\pi}_{t, \xi(t)} \longrightarrow \hat{\pi}_{0}:=q_{*}\left(\Pi_{0}\right) \in \mathfrak{X}^{2}\left(\mathbb{C P}^{n}\right)
$$

\section{5}

\section{Função $\xi(t)$ e Interpretação Geométrica}

$\mathrm{Na}$ seção anterior construímos família de bivetores a dois parêmetros $\Pi_{t, \xi} \in \mathfrak{X}^{2}\left(\mathbb{C}^{n} \backslash\{0\}\right)$. Vamos agora expressar $\xi$ em função de $t$ utilizando-se aspectos geométricos. Temos família de bivetores:

$$
\left\{\Pi_{t, \xi}\right\} \text {, onde } t \in(0,1], \xi \in(-\infty, 0)^{* *}
$$

Podemos normalizar mapa momento para

$$
\hat{\mu}_{t}:=\frac{1}{2} \frac{1}{1-t} \ln \left(\frac{r_{t}\left(z_{1}\right) \cdots r_{t}\left(z_{n}\right)}{t^{n}}\right)
$$

e nossa família de bivetores torna-se:

$$
\left\{\Pi_{t, \zeta}\right\} \text {, onde } t \in(0,1], \zeta \in(0, \infty)
$$


juntamente a função $\theta$ torna-se $s: \theta(t, \xi, z) \rightsquigarrow s(t, \zeta, z)$. Seja $\alpha: \mathbb{C}^{n} \longrightarrow \mathbb{R}^{n}$ dada por

$$
\alpha\left(z_{1} \cdots, z_{n}\right) \mapsto\left(\left|z_{1}\right|^{2}, \cdots,\left|z_{n}\right|^{2}\right)
$$

com imagem no quadrante positivo $Q \subset \mathbb{R}^{n}$.

$$
\frac{t}{s^{2}}=\alpha^{*}\left(\frac{t}{\sigma}\right)
$$

Consideremos $\left(x_{1}, \ldots, x_{n}\right)$ coordenadas de $Q \subset \mathbb{R}^{n}$ e definimos duas famílias de conjuntos:

$$
\begin{aligned}
\Sigma_{t, \zeta} & :=\left\{\left(x_{1}, \ldots, x_{n}\right) \in Q: x_{1} \cdots x_{n}=K(t, \zeta):=t^{n} e^{2(1-t) \zeta}\right\} \\
L_{t, r} & :=\left\{(t, \cdots, t)+\sigma(1-t)\left(r_{1}, \cdots, r_{n}\right) \in Q: \sigma>0\right\}
\end{aligned}
$$

Notemos que dados $t \in(0,1], \zeta>0$ e $0 \neq z \in \mathbb{C}^{n}$ temos:

$$
s z \in \mu_{t}^{-1}(\zeta)
$$

se, e somente, se

$$
\Sigma_{t, \zeta} \cap L_{t, r}=\{(t, \cdots, t)+s(1-t) \alpha(z)\}
$$

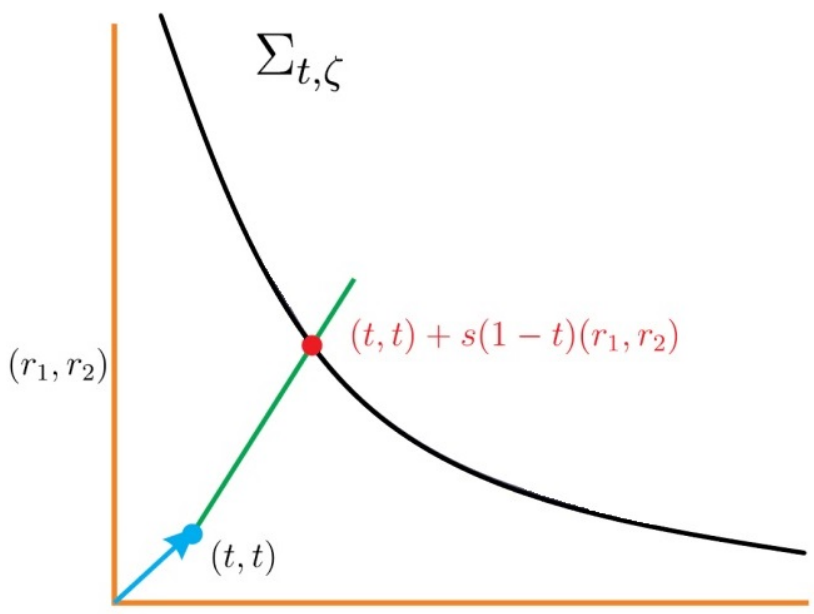

Figura 4.6: Interpretação geométrica

Fazendo escolha de $\zeta(t):=\frac{1}{2} \frac{1}{1-t} \ln \left(\frac{1}{t^{n}}\right)$ resulta que a família de superfícies 
se mantém a mesma e estende-se a $t=0$ :

$$
\Sigma_{t, \zeta(t)}=\left\{\left(x_{1}, \ldots, x_{n}\right) \in Q: x_{1} \ldots x_{n}=1\right\}=: \Sigma_{0}
$$

Para conpactos de $Q \backslash\left\{x_{1} \cdots x_{n} \neq 0\right\}$ temos $s(t, \zeta(t), r)>0$ é contínua e estende-se a $t=0$ e não assumindo valor zero em $t=0$. Logo:

$$
\frac{t}{s(t, \zeta(t), r)} \longrightarrow 0, \text { quando } t \rightarrow 0^{+}
$$

\section{6}

\section{Poisson Comutativo}

O fibrado $q: \mathbb{C}^{n} \backslash\{0\} \longrightarrow \mathbb{C P}^{n-1}$ induz subfibrado $V \in \Gamma\left(T \mathbb{C}^{n}\right)$ dado por $V:=\operatorname{ker}\left(q_{*}\right)$. Por $\mathbb{C}^{n}$ ser variedade hermitiana temos conexão de Eresmann natural dada por $H:=V^{\perp_{\mathbb{C}}}$. Sem grandes dificuldades podemos ver que $V$ é gerado pelo campo de Euler e seu rotacional, $E$ e $R=J(E)$. Chamaremos $V$ e $H$ de parte vertical e parte horizontal, respectivamente. Como mencionado anteriormente a família t-paramétrica $\Pi_{t, \xi(t)}$ não se trata de uma deformação trivial, pois $\hat{\pi}_{t, \xi}$ não comuta com $\pi_{0}$. Para isso basta mostrar que $\left[\Pi_{1, \xi}, \Pi_{0}\right]$ tem componentes horizontais não-nulas.

Vamos lembrar o colchete de Schouten. Seja $\left(U, x_{1}, \ldots, x_{n}\right)$ carta de $M$ e sejam $A \in \mathfrak{X}^{a}(M)$ e $B=\in \mathfrak{X}^{b}(M)$ multicampos. Localmente temos:

$$
A=\sum_{i_{1}<\cdots<i_{a}} A_{i_{1}, \ldots, i_{a}} \frac{\partial}{\partial x_{i_{1}}} \wedge \cdots \wedge \frac{\partial}{\partial x_{i_{a}}}=\sum_{i_{1}, \ldots, i_{a}} A_{i_{1}, \ldots, i_{a}} \zeta_{i_{1}} \ldots \zeta_{i_{a}}
$$

e

$$
A=\sum_{i_{1}<\cdots<i_{a}} A_{i_{1}, \ldots, i_{a}} \frac{\partial}{\partial x_{i_{1}}} \wedge \cdots \wedge \frac{\partial}{\partial x_{i_{a}}}=\sum_{i_{1}, \ldots, i_{a}} A_{i_{1}, \ldots, i_{a}} \zeta_{i_{1}} \ldots \zeta_{i_{a}}
$$

$\log \mathrm{O}$

$$
\left.[A, B]\right|_{U}=\sum_{i} \frac{\partial A}{\partial \zeta_{i}} \wedge \frac{\partial B}{\partial x_{i}}-(-1)^{(a-1)(b-1)} \sum_{i} \frac{\partial B}{\partial \zeta_{i}} \wedge \frac{\partial A}{\partial x_{i}}
$$

onde $\frac{\partial}{\partial \zeta_{i}}$ é a derivação formal. Mais detalhes ver em [16]. Em coordenadas holomorfas $z_{1}, \ldots, z_{n}$ o colchete de Schouten torna-se:

$[A, B]=\sum_{i=1}^{n}\left(\frac{\partial A}{\partial \eta_{i}} \wedge \frac{\partial B}{\partial z_{i}}+\frac{\partial A}{\partial \bar{\eta}_{i}} \wedge \frac{\partial B}{\partial \overline{z_{i}}}\right)-(-1)^{(a-1)(b-1)} \sum_{i=1}^{n}\left(\frac{\partial B}{\partial \eta_{i}} \wedge \frac{\partial A}{\partial z_{i}}+\frac{\partial B}{\partial \bar{\eta}_{i}} \wedge \frac{\partial A}{\partial \overline{z_{i}}}\right)$ onde $\frac{\partial}{\partial \eta_{j}}$ e $\frac{\partial}{\partial \bar{\eta}_{j}}$ são as derivações formais de $\frac{\partial}{\partial z_{j}}$ e $\frac{\partial}{\partial \bar{z}_{j}}$, respectivamente.

Antes de prossegir vamos fazer um esclarecimento breve sobre a obtenção de $\Pi_{1, \xi}$. Primeiramente descobrimos qual a componente vertical $\Pi_{1}=\Pi_{s t d}$, mais 
uma vez sabemos que que a parte vertical $V=\operatorname{Ker}\left(q_{*}\right)$ é gerada pelo campo de Euler e seu rotacional, portanto:

$$
\Pi_{1}^{V}=g E \wedge R, g \in C^{\infty}\left(\mathbb{C}^{n}\right)
$$

definindo $\alpha:=\iota_{E} \omega_{s t d}$ e $\beta:=\iota_{R} \omega_{s t d}$, podemos fazer:

$$
\left\{\begin{array}{l}
\Pi_{1}^{V}(\alpha, \beta)=|z|^{2} \\
E \wedge R(\alpha, \beta)=|z|^{4}
\end{array} \longrightarrow g(z)=\frac{1}{|z|^{2}}\right.
$$

e descobrimos parte vertical $\Pi_{1}^{V}$. Portanto a parte horizontal de $\Pi_{1}$ é dada por $\Pi_{1}^{H}:=\Pi_{1}-\Pi_{1}^{V}$. Dado $\xi$ e fazendo primeira construção da seção 4.4 obtemos:

$$
\begin{aligned}
\Pi_{1, \xi} & =\left(g_{\theta}\right)_{*, f_{\theta}(z)}\left(\Pi_{1}^{V}\left(f_{\theta}(z)\right)\right) \\
& =\frac{i}{\xi} \sum_{j=1}^{n}\left(-\sum_{k \neq j} z_{k} \overline{z_{k}}\right) \frac{\partial}{\partial z_{j}} \wedge \frac{\partial}{\partial \overline{z_{j}}}+\frac{i}{\xi} \sum_{j<k}\left(z_{j} \overline{z_{k}} \frac{\partial}{\partial z_{j}} \wedge \frac{\partial}{\partial \overline{z_{k}}}+z_{k} \overline{z_{j}} \frac{\partial}{\partial z_{k}} \wedge \frac{\partial}{\partial \overline{z_{j}}}\right)
\end{aligned}
$$

Pois bem, sendo $\Pi_{0}=-2 i \sum_{j=1}^{n} z_{j} \overline{z_{j}} \frac{\partial}{\partial z_{j}} \wedge \frac{\partial}{\partial \bar{z}_{j}}$, vamos verificar que $\left[\Pi_{0}, \Pi_{1, \xi}\right] \neq 0$. Agora o trabalho árduo. Vamos calcular

$\left[\Pi_{0}, \Pi_{1, \xi}\right]=\sum_{i=1}^{n}\left(\frac{\partial \Pi_{0}}{\partial \eta_{i}} \wedge \frac{\partial \Pi_{1, \xi}}{\partial z_{i}}+\frac{\partial \Pi_{0}}{\partial \bar{\eta}_{i}} \wedge \frac{\partial \Pi_{1, \xi}}{\partial \overline{z_{i}}}\right)+\sum_{i=1}^{n}\left(\frac{\partial \Pi_{1, \xi}}{\partial \eta_{i}} \wedge \frac{\partial \Pi_{0}}{\partial z_{i}}+\frac{\partial \Pi_{1, \xi}}{\partial \overline{\eta_{i}}} \wedge \frac{\partial \Pi_{0}}{\partial \overline{z_{i}}}\right)$ por partes!

$$
\text { • } \sum_{i=1}^{n}\left(\frac{\partial \Pi_{0}}{\partial \eta_{i}} \wedge \frac{\partial \Pi_{1, \xi}}{\partial z_{i}}+\frac{\partial \Pi_{0}}{\partial \bar{\eta}_{i}} \wedge \frac{\partial \Pi_{1, \xi}}{\partial \bar{z}_{i}}\right)
$$

para $j \in\{1, \ldots, n\}$ temos:

$$
\frac{\partial \Pi_{0}}{\partial \eta_{j}} \wedge \frac{\partial \Pi_{1, \xi}}{\partial z_{j}}=\frac{2}{\xi} z_{j} \overline{z_{j}} \sum_{k \neq j}^{n}\left(\overline{z_{j}} \frac{\partial}{\partial \overline{z_{j}}} \wedge \frac{\partial}{\partial z_{k}} \wedge \frac{\partial}{\partial \overline{z_{k}}}+\overline{z_{k}} \frac{\partial}{\partial z_{j}} \wedge \frac{\partial}{\partial \overline{z_{j}}} \wedge \frac{\partial}{\partial \overline{z_{k}}}\right)
$$

e

$$
\frac{\partial \Pi_{0}}{\partial \overline{\eta_{i}}} \wedge \frac{\partial \Pi_{1, \xi}}{\partial \overline{z_{i}}}=\frac{2}{\xi} z_{j} \overline{z_{j}} \sum_{k \neq j}^{n}\left(-z_{j} \frac{\partial}{\partial z_{j}} \wedge \frac{\partial}{\partial z_{k}} \wedge \frac{\partial}{\partial \overline{z_{k}}}-z_{k} \frac{\partial}{\partial z_{j}} \wedge \frac{\partial}{\partial \overline{z_{j}}} \wedge \frac{\partial}{\partial z_{k}}\right)
$$

Portanto

$$
\begin{gathered}
\sum_{i=1}^{n}\left(\frac{\partial \Pi_{0}}{\partial \eta_{i}} \wedge \frac{\partial \Pi_{1, \xi}}{\partial z_{i}}+\frac{\partial \Pi_{0}}{\partial \overline{\eta_{i}}} \wedge \frac{\partial \Pi_{1, \xi}}{\partial \overline{z_{i}}}\right)= \\
=\frac{2}{\xi} \sum_{j=1}^{n} z_{j} \overline{z_{j}} \sum_{k \neq j}\left(\overline{z_{j}} \frac{\partial}{\partial \overline{z_{j}}} \wedge \frac{\partial}{\partial z_{k}} \wedge \frac{\partial}{\partial \overline{z_{k}}}+\overline{z_{k}} \frac{\partial}{\partial z_{j}} \wedge \frac{\partial}{\partial \overline{z_{j}}} \wedge \frac{\partial}{\partial \overline{z_{k}}}-z_{j} \frac{\partial}{\partial z_{j}} \wedge \frac{\partial}{\partial z_{k}} \wedge \frac{\partial}{\partial \overline{z_{k}}}-z_{k} \frac{\partial}{\partial z_{j}} \wedge \frac{\partial}{\partial \overline{z_{j}}} \wedge \frac{\partial}{\partial z_{k}}\right)
\end{gathered}
$$

Analogamente temos: 
$\bullet \sum_{i=1}^{n}\left(\frac{\partial \Pi_{1, \xi}}{\partial \eta_{i}} \wedge \frac{\partial \Pi_{0}}{\partial z_{i}}+\frac{\partial \Pi_{1, \xi}}{\partial \overline{\eta_{i}}} \wedge \frac{\partial \Pi_{0}}{\partial \overline{z_{i}}}\right)=\frac{2}{\xi} \sum_{j=1}^{n} z_{j} \overline{z_{j}} \sum_{k \neq j}\left(z_{k} \frac{\partial}{\partial z_{k}} \wedge \frac{\partial}{\partial z_{j}} \wedge \frac{\partial}{\partial \overline{z_{j}}}-\overline{z_{k}} \frac{\partial}{\partial \overline{z_{k}}} \wedge \frac{\partial}{\partial z_{j}} \wedge \frac{\partial}{\partial \overline{z_{j}}}\right)$ e então $\left[\Pi_{0}, \Pi_{1, \xi}\right]=\frac{2}{\xi} \sum_{j=1}^{n} z_{j} \overline{z_{j}} \sum_{k \neq j} A_{j k}$, onde:

$$
\begin{aligned}
A_{j k}= & \overline{z_{j}} \frac{\partial}{\partial \overline{z_{j}}} \wedge \frac{\partial}{\partial z_{k}} \wedge \frac{\partial}{\partial \overline{z_{k}}}+\overline{z_{k}} \frac{\partial}{\partial z_{j}} \wedge \frac{\partial}{\partial \overline{z_{j}}} \wedge \frac{\partial}{\partial \overline{z_{k}}}-z_{j} \frac{\partial}{\partial z_{j}} \wedge \frac{\partial}{\partial z_{k}} \wedge \frac{\partial}{\partial \overline{z_{k}}} \\
& -z_{k} \frac{\partial}{\partial z_{j}} \wedge \frac{\partial}{\partial \overline{z_{j}}} \wedge \frac{\partial}{\partial z_{k}}+z_{k} \frac{\partial}{\partial z_{k}} \wedge \frac{\partial}{\partial z_{j}} \wedge \frac{\partial}{\partial \overline{z_{j}}}-\overline{z_{k}} \frac{\partial}{\partial \overline{z_{k}}} \wedge \frac{\partial}{\partial z_{j}} \wedge \frac{\partial}{\partial \overline{z_{j}}}
\end{aligned}
$$

simplificando os $A_{j k}$ concluímos que

$$
\left[\Pi_{0}, \Pi_{1, \xi}\right]=\frac{2}{\xi} \sum_{j=1}^{n} z_{j} \overline{z_{j}} \sum_{k \neq j}\left(\overline{z_{j}} \frac{\partial}{\partial \overline{z_{j}}}-z_{j} \frac{\partial}{\partial z_{j}}\right) \frac{\partial}{\partial z_{k}} \wedge \frac{\partial}{\partial \overline{z_{k}}}
$$

Vamos agora verificar que $\left[\Pi_{0}, \Pi_{1, \xi}\right] \in \mathfrak{X}^{3}\left(\mathbb{C}^{n}\right)$ é campo não nulo sobre distribuição $H$. Assumimos que $n \geq 4$ e $z=\left(z_{1}, \ldots, z_{n}\right)$ com $z_{1} \neq 0$. Definimos para $j=2, \ldots, n$ o ponto $v_{j}:=\left(\overline{z_{j}}, 0, \ldots,-\overline{z_{1}}, \ldots, 0\right)$, onde $-\overline{z_{1}}$ está na posição $j$, e o campo

$$
H^{j}(z):=\left.\frac{d}{d t}\right|_{t=0}\left(z+t v_{j}\right)=\overline{z_{j}} \frac{\partial}{\partial z_{1}}+z_{j} \frac{\partial}{\partial \overline{z_{1}}}-\overline{z_{1}} \frac{\partial}{\partial z_{j}}-z_{1} \frac{\partial}{\partial \overline{z_{j}}}
$$

Notemos que $\left\{H^{2}(z), \ldots, H^{n}(z)\right\}$ são linearmente independentes em $\mathbb{C}^{n} \backslash\left\{z_{1}=\right.$ $0\}$. Desta forma, definimos

$$
\alpha_{j}:=\imath_{H^{j}} \omega_{s t d}=\frac{i}{2}\left(-z_{j} d z_{1}+\overline{z_{j}} d \overline{z_{1}}+z_{1} d z_{j}-\overline{z_{1}} d \overline{z_{j}}\right)
$$

$\operatorname{ker}\left(\alpha_{j}\right)=\left\{H^{j}\right\}^{\omega_{s t d}}$ e, em particular, $\alpha_{j}$ não se anula em $J\left(H^{j}\right), J$ estrutura complexa. Aplicando:

$$
\left[\Pi_{0}, \Pi_{1, \xi}\right]\left(\alpha_{2}, \alpha_{3}, \alpha_{3}\right)=\frac{2}{\xi} \sum_{j=1}^{n} z_{j} \overline{z_{j}} \sum_{k \neq j}\left(\overline{z_{j}} \frac{\partial}{\partial \overline{z_{j}}}-z_{j} \frac{\partial}{\partial z_{j}}\right) \frac{\partial}{\partial z_{k}} \wedge \frac{\partial}{\partial \overline{x_{k}}}\left(\alpha_{2}, \alpha_{3}, \alpha_{3}\right)
$$

Para $k \neq 1$ temos que $\left(\overline{z_{j}} \frac{\partial}{\partial \overline{z_{j}}}-z_{j} \frac{\partial}{\partial z_{j}}\right) \wedge \frac{\partial}{\partial z_{k}} \wedge \frac{\partial}{\partial \overline{z_{k}}}\left(\alpha_{2}, \alpha_{3}, \alpha_{3}\right)=0$, nos casos restantes resulta:

- $j=2, k=1$

$$
\left(\overline{z_{2}} \frac{\partial}{\partial \overline{z_{2}}}-z_{2} \frac{\partial}{\partial z_{2}}\right) \wedge \frac{\partial}{\partial z_{1}} \wedge \frac{\partial}{\partial \overline{z_{1}}}\left(\alpha_{2}, \alpha_{3}, \alpha_{3}\right)=-\left(\overline{z_{1} z_{2}}+z_{1} z_{2}\right)\left(\overline{z_{3}} z_{4}-z_{3} \overline{z_{4}}\right)=: B_{2}
$$


- $j=3, k=1$

$\left(\overline{z_{3}} \frac{\partial}{\partial \overline{z_{3}}}-z_{3} \frac{\partial}{\partial z_{3}}\right) \wedge \frac{\partial}{\partial z_{1}} \wedge \frac{\partial}{\partial \overline{z_{1}}}\left(\alpha_{2}, \alpha_{3}, \alpha_{3}\right)=-\left(\overline{z_{1} z_{3}}+z_{1} z_{3}\right)\left(\overline{z_{4}} z_{2}-z_{4} \overline{z_{2}}\right)=: B_{3}$

- $j=4, k=1$

$\left(\overline{z_{4}} \frac{\partial}{\partial \overline{z_{4}}}-z_{4} \frac{\partial}{\partial z_{4}}\right) \wedge \frac{\partial}{\partial z_{1}} \wedge \frac{\partial}{\partial \overline{z_{1}}}\left(\alpha_{2}, \alpha_{3}, \alpha_{3}\right)=-\left(\overline{z_{1} z_{4}}+z_{1} z_{4}\right)\left(\overline{z_{2}} z_{3}-z_{2} \overline{z_{3}}\right)=: B_{4}$

Logo

$$
\left[\Pi_{0}, \Pi_{1, \xi}\right]\left(\alpha_{2}, \alpha_{3}, \alpha_{3}\right)=-\frac{1}{2 \xi} \sum_{j=2}^{4} z_{j} \overline{z_{j}} B_{j} \not \equiv 0
$$

$q: \mathbb{C}^{n} \backslash\{0\} \longrightarrow \mathbb{C P}^{n-1}$ projeção canônica

$$
\Rightarrow q_{*}\left[\Pi_{0}, \Pi_{1, \xi}\right]=\left[q_{*} \Pi_{0}, q_{*} \Pi_{1, \xi}\right] \neq 0
$$

Para o caso $n=3$ escolhemos $\alpha_{2}:=\imath_{H^{2}} \omega_{s t d}, \alpha_{3}:=\imath_{J\left(H^{2}\right)} \omega_{s t d}, \alpha_{4}=\imath_{H^{3}} \omega_{s t d}$ 


\section{Referências bibliográficas}

[1] Alan Weinstein The Local Structure of Poisson Manifolds J. Differential Geometry, 18 (1983) 523557.

[2] J.E. Marsden and T.S. Ratiu, Reduction of Poisson manifolds, Lett. Math. Phys. 11 (1986), no. 2, 161-169.

[3] Victor Guillemin, Eva Miranda, Geoffrey Scott and Ana Rita Pires Toric actions on b-symplectic manifolds10.1093/imrn/rnu108, july, 2015

[4] J.-P. Ortega, The symplectic reduced spaces of a Poisson action, C. $R$. Acad. Sci. Paris Sér. I Math. 334 (2002), 999-1004.

[5] Victorw. Guillemin, Eva Miranda, and Jonathan Weitsman On geometric quantization of b-symplectic manifolds https://arxiv.org/abs/1608.08667v1

[6] Jiang-Hua Lu Classical dynamical r-matrices and homogeneous Poisson structures on $G=H$ and $K=T$ University of Arizona, Tucson, AZ 85721

[7] Arlo Cane Toric Poisson Structures Moscow Mathematical Journal, April-June 2011

[8] Nobutada Nakanishi Poisson Cohomology of Plane Quadratic Poisson Structures Publ. RIMS, Kyoto Univ. 1997

[9] Schwarz, Gerald W. Smooth functions invariant under the action of a compact Lie group. Topology 14 (1975), 63-68.

[10] J.-P. Ortega and T.S. Ratiu, Momentum Maps and Hamiltonian Reduction, Progress in Mathematics, volume 222. Birkhaüser Verlag, 2004. $375-422$.

[11] A. Cannas da Silva, Lectures on symplectic geometry, Lecture Notes in Mathematics, Published by Springer-Verlag as number 1764, 2006

[12] A. Cannas da Silva, Symplectic Toric Manifolds 2001

[13] D. McDuff, D. Salamon Introduction to symplectic topology. Second edition. Oxford Mathematical Monographs. The Clarendon Press, Oxford University Press, New York, 1998. 
[14] André Lichnerowicz Les varietes de Poisson et leurs algebres de Lie associees Journal of Differential Geometry

[15] V. Guillemin and S. Sternberg, Convexity properties of the moment mapping, Invent. Math. 67 (1982), no. 3, 491-513.

[16] Dufour, J.P. e Zung, N.T. Poisson structures and their normal forms, Progress in Mathematics, vol. 242, Birkhäuser Basel, 2005.

[17] Bursztyn, H. e Macarini, L. Introdução à Geometria Simplética, XIV Escola de Geometria Diferencial, IMPA, Rio de Janeiro, 2006.

[18] Lee, J.M. Introduction to Smooth Manifolds, vol. 218, Springer Verlag, 2003

[19] Marsden, J.E. e Ratiu, T.S. Introduction to mechanics and symmetry: a basic exposition of classical mechanical systems, vol. 17. Springer Verlag, 1999.

[20] Fernandes, R. L. e Marcut, I.. Lectures on poisson geometry, 2014. Accesed on march 2018

[21] Lee, J. M., Manifolds and Differential Geometry Graduate Studies in Mathematics Volume 107, American Mathematical Society, 2009

[22] Tu, L. W., An introduction to manifolds, Second Edition, Springer, 2010. 